\title{
Dynamic Primitives Facilitate Manipulating a Whip
}

\author{
by \\ Moses C. Nah
}

Bachelor of Science in Mechanical and Aerospace Engineering

Seoul National University (2018)

Submitted to the Department of Mechanical Engineering

in partial fulfillment of the requirements for the degree of

Master of Science in Mechanical Engineering

at the

MASSACHUSETTS INSTITUTE OF TECHNOLOGY

May 2020

(c) Massachusetts Institute of Technology 2020. All rights reserved.

Author

Department of Mechanical Engineering

May 15, 2020

Certified by

Neville Hogan

Sun Jae Professor of Mechanical Engineering Thesis Supervisor

Accepted by

Nicolas G. Hadjiconstantinou

Chairman, Department Committee on Graduate Students 


\title{
Dynamic Primitives Facilitate Manipulating a Whip
}

\author{
by \\ Moses C. Nah
Submitted to the Department of Mechanical Engineering
on May 15, 2020, in partial fulfillment of the
requirements for the degree of
Master of Science in Mechanical Engineering

\begin{abstract}
Human dexterity far exceeds that of modern robots, despite a much slower neuromuscular system. Understanding how this is accomplished may lead to improved robot control. The slow neuromuscular system of humans implies that prediction based on some form of internal model plays a prominent role. However, the nature of the model itself remains unclear. To address this problem, we focused on one of the most complex and exotic tools humans can manipulate - a whip. We tested (in simulation) whether a distant target could be reached with a whip using a (small) number of dynamic primitives, whose parameters could be learned through optimization. This approach was able to manage the complexity of an (extremely) high degree-of-freedom system and discovered the optimal parameters of the upper-limb movement that achieved the task. A detailed model of the whip dynamics was not needed for this approach, which thereby significantly relieved the computational burden of task representation and performance optimization. These results support our hypothesis that composing control using dynamic motor primitives may be a strategy which humans use to enable their remarkable dexterity. A similar approach may contribute to improved robot control.
\end{abstract}

Thesis Supervisor: Neville Hogan

Title: Sun Jae Professor of Mechanical Engineering 


\section{Acknowledgments}

It is my pleasure to acknowledge the people who have been assisting me during my Master's degree program at MIT. This thesis is a product of numerous inputs, influences, contributions and quite simply help from many people: my advisor, lab members, friends and my family. I shall be glad to think that this thesis may at least in part repay their invaluable efforts. Without their generous help and unwavering support, this thesis would never have seen the day.

First of all, I must express my deepest appreciation to my advisor Prof. Neville Hogan. It is my true privilege to have him as my advisor and mentor. This thesis would definitely not have been possible without his keen insight, untiring support and intellectual stimuli. His incisive comments continuously inspired and sharpened my thinking, pushing me to reconsider any conclusions which I might had reached. His guidance continues to be the source of my internal growth.

I would also like to thank all of the past and current members of MIT Newman Lab: Johannes Lachner, Jongwoo Lee, Meghan Huber, Davi da Silva, James Hermus, David Verdi, Logan Leahy, Michael West, Kaymie Shiozawa, Stephan Stansfield and Kuangen Zhang. The innumerable and extensive conversations within our group have helped me strengthen my analytical voice in this thesis. I would further like to thank the Korean community, KGSA-ME, ex-coworkers from NAVER LABS, members from Northeastern University Action Lab and all the people who have been interacting with me during my campus life in different situations.

Outside of our lab, I especially like to thank Danyal Rehman and Ernest Kim, my best friends and life-companion in Cambridge. Directly or indirectly, we have took most of our steps together during the life in Cambridge, and the warm support which I have received always assisted me to step forward. I believe that the experiences we shared together will always lead us to mutual growth and happiness, and will eventually guide us to the place that we have been longing to reach.

Special thanks to Ann, Ron, Lia and Wyn, for helping me seamlessly adapt to this new environment, and for welcoming me with their warm hospitality. The time 
that we have shared together: foliage, flower-peeping, kayaking and others, enriched my life in Boston. I have no doubt that restaurant Giacomo's at South End, where we often visited for dinner, is and will always be my favorite restaurant in Boston.

Last, but certainly not least, I would like to express my deepest gratitude to my family. The love from my family is the life's greatest blessing. My uncle, Bongwook Lee and my nephew Sam (Seojun Lee), who live in Boston. It is hard to express in words how grateful and blessed I am to have my family in the same city. My uncle's unparalleled experience in computer engineering have enormously helped my research. My older brother Joseph Nah and my sister-in-law Yerim Yoon. Their warm hospitality and mature guidance have been invaluable. Their wise advice always assisted me to overcome the obstacles in my path. My mom and dad, whom had brought me to this life. I am eternally grateful and privileged to be your son. Your kindness, openness, tolerance, generosity, help and love have made me who I am. You taught me that learning should be a joyful experience and must be pursued for its own sake. The general support and life philosophy you emphasized have helped me become a higher self everyday. I will simply never be able to thank you enough.

This research was performed in the Eric P. and Evelyn E. Newman Laboratory for Biomechanics and Human Rehabilitation at the Massachusetts Institute of Technology. The research was supported by National Science Foundation Mind, Machine and Motor Nexus (M3X) Grant No. 1826097, No. 1825942, and by National Institutes of Health Research Project Grant (R01) No. HD087089 


\section{Contents}

1 Introduction $\quad 23$

1.1 The Paradox of Human Performance . . . . . . . . . . . . . . . 23

1.2 Internal Models in Human Motor Control . . . . . . . . . . . . . . . . 24

1.3 Why study a Whip? - Complexity is Informative . . . . . . . . . . 25

1.4 Dynamic Motor Primitives Hypothesis . . . . . . . . . . . . . 26

1.5 Overview of Thesis . . . . . . . . . . . . . . . . 28

2 Dynamic Motor Primitives $\quad 29$

2.1 Submovements . . . . . . . . . . . . . . . . . . . . 29

2.2 Oscillations . . . . . . . . . . . . . . . . . . . 32

2.3 Mechanical Impedances . . . . . . . . . . . . . . . . . . . . 33

2.4 Norton Equivalent Network Model . . . . . . . . . . . . . . . . 36

3 Modeling $\quad 39$

3.1 Whip Model Type 1 - Planar . . . . . . . . . . . . . . . . . . 39

3.2 Whip Model Type 2 -Spatial . . . . . . . . . . . . . . . . . 40

3.3 Upper-limb Model Type 1 - Planar . . . . . . . . . . . . . . 41

3.4 Upper-limb Model Type 2 - Spatial . . . . . . . . . . . . . . . . . . 42

4 Validation of the MuJoCo simulator $\quad 45$

4.1 Methods . . . . . . . . . . . . . . . . 45

4.1.1 Method 1 - Initial Condition Response . . . . . . . . . . . . . 45

4.1.2 Method 2 - Displacement Function Excitation . . . . . . . . 46 
4.2 Results. . . . . . . . . . . . . . . . . . . . 48

4.2.1 Method 1 - Initial Condition Response . . . . . . . . . . 48

4.2.2 Method 2 - Displacement Function Excitation . . . . . . . 50

4.3 Conclusion . . . . . . . . . . . . . . . . . . 51

$5 \quad$ Analysis of a Linearized Whip Model $\quad 53$

5.1 Method ............................. 54



5.2 .1 Pole-Zero Analysis . . . . . . . . . . . . . . 55

$5.2 .2 \quad$ Spectral Analysis . . . . . . . . . . . . . . . 55

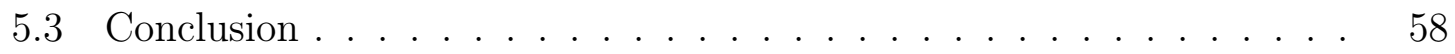

6 Experimental Estimation of Whip Parameters 59

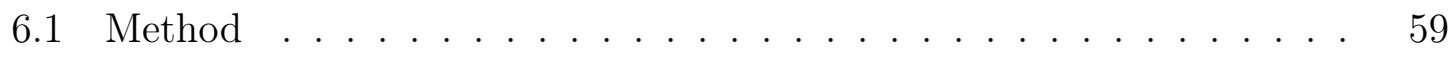

6.2 Result . . . . . . . . . . . . . . . . 63

$\begin{array}{lll}7 & \text { 2D Planar Whip Task } & 67\end{array}$

7.1 Modeling. . . . . . . . . . . . . . . . . . . . . 67

7.2 Control of the upper-limb . . . . . . . . . . . . . . 68

7.2 .1 Impedance Controller . . . . . . . . . . . . . . . 68

7.2 .2 Zero-Torque Trajectory . . . . . . . . . . . . . . . . 71

7.2 .3 Implementation . . . . . . . . . . . . . . . . 73

7.3 Optimization Method . . . . . . . . . . . . . . 74

7.4 Optimization and Simulation Results . . . . . . . . . 76

7.5 Robustness Analysis of the Optimal Solution . . . . . . . . . . 80

7.5 .1 Method ..................... 80

7.5 .2 Result . . . . . . . . . . . . . . . . . 81

7.6 Optimization of the Experimentally-fitted Whip Model . . . . . . 86

8 Spatial Whip Task $\quad 89$

8.1 Modeling . . . . . . . . . . . . . . . . . . . . . . . . 89

8.2 Control of the upper-limb . . . . . . . . . . . . . 91 
8.2.1 Impedance Controller . . . . . . . . . . . . . . . . . . . 91

8.2.2 Zero-Torque Trajectory . . . . . . . . . . . . . . . . . . 92

8.3 Optimization Method . . . . . . . . . . . . . . . . . . 93

8.4 Optimization and Simulation Results . . . . . . . . . . . . 94

9 Conclusions and Discussion 103

9.1 Relation to Prior Work . . . . . . . . . . . . . . . . . . . . . . . . 104

9.2 Limitations and Future Direction _ . . . . . . . . . . . 105

9.3 Conclusion . . . . . . . . . . . . . . . . . . . . . . . . 106

$\begin{array}{ll}\text { A Dynamics of the Multi-Pendulum } & 107\end{array}$

A.1 Equation of Motion of the Multi-Pendulum . . . . . . . . . . 107

A.2 Linearization of the Multi-Pendulum Model . . . . . . . . . . . 112

A.3 Coordinate Transformation . . . . . . . . . . . . . . . . . . . . . 114

$\begin{array}{ll}\text { B Calculus of Variations } & 117\end{array}$

B.1 Problem Statement . . . . . . . . . . . . . . . . . . 117

B.2 Euler-Lagrange Equation . . . . . . . . . . . . . . . . . 118

B.3 Euler-Poisson Equation . . . . . . . . . . . . . . . 120

B.4 Derivation of Minimum-Jerk-Trajectory . . . . . . . . . . . 120

$\begin{array}{ll}\text { C Comparison of Nlopt Algorithms } & 123\end{array}$

C.1 Comparison and Results . . . . . . . . . . . . . . . . . . . . 124

C.2 DIRECT-L Algorithm . . . . . . . . . . . . . . 126 


\section{List of Figures}

1-1 Three dynamic motor primitives - submovements, oscillations and mechanical impedances. . . . . . . . . . . . . . . . . . . 27

2-1 Plot of a representative single submovement with a unimodal speed profile $\dot{x}_{j}(t)$. The basis function $\sigma(t)$ is plotted as a dashed line. $\hat{v}$ is the peak speed of the submovement; $D$ is the duration of the submovement; $t_{p}$ is the time when the peak happened. . . . . . . . . . .

2-2 Plot of 2 superimposed submovements. Individual submovements are plotted as dashed lines. $b, d$ and $\hat{v}_{j}$ are the start time, duration and speed amplitude of $j^{\text {th }}$ coordinate for each submovement. . . . . . . .

2-3 Diagram of a Norton equivalent network model of the human motor control system. A nonlinear equivalent network relating motion planning in the information domain with interactive dynamics in the energy domain [Hogan, 2013]. . . . . . . . . . . . . . . . . .

3-1 (a) Planar sub-model of the $N$-node whip model. The blue filled circle depicts the (ideal) point mass $m$ of the sub-model. The mass $m$ was attached to the grey massless cylinder with length $l$. The swirl icon on the pivot symbolizes the linear torsional spring $k$ and linear torsional damper $b$. (b) $N$ identical sub-models comprised the $N$-node whip model. . . . . . . . . . . . . . . . . . 
3-2 A 3D spatial model of the $N$-node whip model. Axes of rotation are visualized as a bullet shape. Each node of the whip had 2 degreesof-freedom. The 3D whip model was modeled and rendered with the MuJoCo simulator. . . . . . . . . . . . . . . . .

3-3 The two-bar planar upper-limb model. $x^{\prime}, y^{\prime}$ and $z^{\prime}$ denote the axes of the principal moments of inertia for each limb segment. Values and description of the mathematical notation are shown in [Table 3.1]. . .

3-4 A spatial upper-limb model rendered with the MuJoCo simulator. A Cartesian coordinate frame is indicated at the shoulder joint. Joint J1, J2 and J3 were perpendicularly connected to reproduce a 3D shoulder joint movement. The order of rotations of the three shoulder joints were ordered with ascending number, i.e. J1 - J2 - J3. J1, J2 and J3 corresponded to flexion/extension, adduction/abduction and lateral/medial rotation, respectively. Axes of rotation are visualized as a bullet shape. . . . . . . . . . . . . . . . . . .

4-1 The initial condition response method. From a given initial position at time $t=0 \mathrm{~s}$, the lossless whip model was set loose and the energy of the system was monitored. Vector $\boldsymbol{g}$ denotes gravity of the simulation

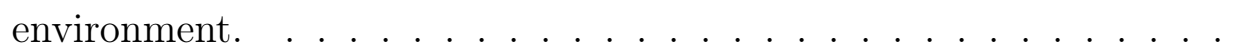

4-2 The displacement function excitation method. The upper graph depicts the representative function profile of displacement function $p(t)$ [Eqn. 4.1]. $t_{0}[s]$ denotes time when the displacement function started and $w[s]$ denotes the duration of the motion. From the equilibrium configuration at rest hanging vertically downward, the whip was excited with function $p(t)$ from time domain $\left[t_{0}, t_{0}+w\right] . . . .$. 
4-3 Time vs. energy plot of a 1-node whip model. The 1-node whip model was initially positioned 3.13-radian from the vertically-down stable equilibrium posture (i.e. 0.01-radian off from the vertically-upward unstable equilibrium posture). Detailed whip parameters: $(N, l, m, k, b)=$ $(1,1,1,0,0) \ldots \ldots \ldots \ldots$

4-4 Plot of total energy vs. time with different lossless whip models. Values for mass $m$, length $l$, torsional stiffness coefficient $k$ and torsional damping coefficient $b$ were fixed, and different values of node number $N$ were tested. The straightened $N$-node whip model was initially positioned 0.3-radian from the vertically down equilibrium posture, and was released to freely oscillate. Whip parameters: $(l, m, k, b)=$ $(0.1,0.1,0,0) \ldots \ldots \ldots \ldots \ldots$

4-5 Numerical dissipation of total energy over time with different lossless whip models. Excitation with displacement function $p(t)$ started at $1.5 \mathrm{~s}$. Values for mass $m$, length $l$, torsional stiffness coefficient $k$ and torsional damping coefficient $b$ were fixed, and different values of node number $N$ were tested. Whip parameters: $(l, m, k, b)=$ $(0.1,0.02,0.05,0)$; displacement function parameters: $\left(A, t_{0}, w\right)=$ $(0.02,1.5,0.1)[$ Eqn. 4.1] . . . . . . . . . . . . . .

5-1 Plot of the (complex) eigenvalues of matrix $\boldsymbol{A}$ in the complex plane. The radial grids are numbered in ascending order of distance from the origin. Whip parameters: $(N, l, m, k)=(3,0.1,0.02,0.05) . \ldots 56$ 
5-2 Spectral analysis of a 3-node whip model. First row: time vs. $3^{\text {rd }}$ joint angle plot; second row: Power spectra of the corresponding joint angle. The excitation of the whip model started at $1.5 \mathrm{~s}$. Within the 5 minute long data record, spectral analysis was conducted on a segment of joint angle data from 20s to 60s. Columns show results with different values of joint damping; left to right, $b=0,0.0005$ and 0.001 , respectively. Note the rapid convergence to a low-frequency oscillation with even a modest amount of damping. The vertical dotted lines denote the theoretical frequencies of the eigenmodes. Whip parameters: $(N, l, m, k)$ $=(3,0.1,0.02,0.05)$; displacement function parameters: $\left(A, t_{0}, w\right)$ $=(0.02,1.5,0.1)[$ Eqn. 4.1] . . . . . . . . . . . .

6-1 (a) Illustration of the actual whip. The green-shaded region depicts the handle of the whip; the yellow-shaded region depicts the main part of the whip; the circles attached to the whip illustrate the markers used for position tracking. (b) Customized $1.8 \mathrm{~m}$ bullwhip used for the experiment. In total, 12 markers were mounted on the whip. . . . .

6-2 Plot of the 2D horizontal position of the marker attached at the tip of the whip. The position data of $x$ and $y$ coordinates were adjusted to position the mean value of $x$ and $y$ position at the origin. The blue dot denotes the starting position of the oscillation. The 2D horizontal data were rotated to align the blue marker with the global $x$ axis. . .

6-3 (Left) FFT spectral analysis of the $x$ position of the whip markers. (Right) FFT spectral analysis of the $y$ position of the whip markers. Excluding the markers attached to the handle, 10 markers were numbered in ascending order of distance from the handle. The dominant frequency value was $0.45 \mathrm{~Hz}$ for both cases. . . . . . . . . . . . . . . 
6-4 Plot of the rotated $x$ position data vs. time of the experimental and curve-fit data. The red-dotted line depicts the curve-fit function $c(t)$ [Eqn. 6.1]; the blue-dotted line depicts the corresponding exponential decay curve. Detailed parameters of function $c(t):\left(C, \tau_{\text {decay }}, \phi\right)=$ $(180.07,29.24,-1.062) . \ldots \ldots \ldots$

6-5 Plot of the $x$ position of the tip of the modeled whip vs. time of the MuJoCo and curve-fit data. The yellow-dotted line depicts the curve-fit function $c(t)$ [Eqn. 6.1]; the blue-dotted line depicts the corresponding exponential decay curve. Detailed parameters of function $c(t):\left(C, \tau_{\text {decay }}, f, \phi\right)=(180.19,29.07,0.45,-1.27) . \ldots 66$

6-6 Comparison between the actual whip and the experimentally-fitted whip model of MuJoCo. The $x$ position of the tip of the whip for the MuJoCo model and the actual whip are plotted and compared. The root-mean-square error was $43.65 \mathrm{~mm}$. . . . . . . . . . . . . .

7-1 The model used for the planar whip task. The planar upper-limb and planar whip model were combined. SH and EL denote shoulder and elbow joint, respectively. . . . . . . . . . . . . . .

7-2 The relative angle coordinate used for the planar upper-limb model. Angle $\theta_{1}(\mathrm{rad})$ and $\theta_{2}(\mathrm{rad})$ denote relative angles of the shoulder and elbow joint, respectively. . . . . . . . . . . . . .

7-3 Frames and the imposed forces of the simulation model. . . . . . . . 70

7-4 Plot of the zero-torque trajectory of the shoulder $\phi_{1}(t)$ and elbow $\phi_{2}(t)$ joint. Detailed values for $\phi_{1, \max }$ and $\phi_{2, \max }$ are $1.875 \cdot\left(\phi_{1, f}-\phi_{1, i}\right)$ and 1.875 . $\left(\phi_{2, f}-\phi_{2, i}\right)$, respectively. . . . . . . . . . . . .

7-5 The upper-limb controller represented with the Norton equivalent network model. . . . . . . . . . . . . . . . . . . . . 
7-6 Representative images of the zero-torque trajectory and the actual upper-limb movement. The top row depicts the zero-torque trajectory in joint space coordinates. In the bottom row, the transparent upper-limb depicts the zero-torque position of the upper-limb. $t$ denotes time and $D$ denotes the duration of the single discrete upper-limb movement. ........................ 73

7-7 The 2D planar whip task. . . . . . . . . . . . . . 75

7-8 The position of the target. . . . . . . . . . . . 75

7-9 Time sequence of the simulation of the whip task. (A) Short-whip (B) Medium-whip (C) Long-whip. Each upper-limb movement was generated with the optimal movement parameters, which yielded the minimum distance $L^{*}$ [Table 7.3] . . . . . . . . . . . . . . . . 78

7-10 Optimization results for the three whip models. 600 iterations were conducted with the DIRECT-L algorithm. . . . . . . . . . . . .

7-11 (Top) Number of iterations vs. $L^{*}$ value for the short-whip model. The shaded region depicts $L^{*}$ values lower than $0.05 \mathrm{~m}$. (Bottom) Distribution of the data of the corresponding 5 movement parameters, which resulted in $L^{*}$ values lower than $0.05 \mathrm{~m}$. The shaded region depicts the upper and lower bound of the optimization [Table 7.2]. . . . . . .

7-12 3D plot of the initial shoulder angle $\phi_{1, i}$, initial elbow angle $\phi_{2, i}$ of the zero-torque trajectory vs. output $L^{*}$. The points were evenly sampled with 0.001-radian step size for shoulder and elbow angles. In total, 3362 points were sampled. . . . . . . . . . . . . . .

7-13 (Left) 3D plot of initial shoulder angle $\phi_{1, i}$, initial elbow angle $\phi_{2, i}$ of the zero-torque trajectory vs. output $L^{*}$ with different damping values. (Right) Equivalent graph viewed with a camera located at $\left(44.3242^{\circ}, 29.3597^{\circ}\right)$ in (azimuth-elevation coordinate). As the damping value decreased, the landscape of $L^{*}$ lost its smoothness. The points were evenly sampled with 0.001-radian step size for shoulder and elbow angles. In total, 3362 points were sampled for each value of $b$. . . . . 
7-14 Time sequence of the simulation of the experimentally-fitted whip model. Each upper-limb movement was generated with the optimal movement parameters, which yielded the minimum distance $L^{*}$ [Table

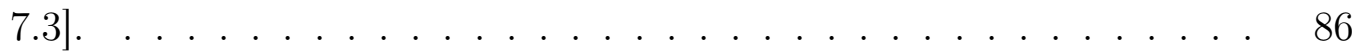

7-15 Optimization results for the experimentally-fitted whip model. 600 iterations were conducted with the DIRECT-L algorithm. . . . . .

8-1 The model used for the spatial whip task. A 4 degrees-of-freedom (DOF) upper-limb and spatial whip model were combined. . . . . .

8-2 The upper-limb and the spatial whip model at an equilibrium posture. The connection of the upper-limb and whip model was achieved by 2 freely-rotating hinge joints perpendicular to each other. Axes of rotation for each joint are visualized as a bullet shapes. . . . . . .

8-3 The target positions depicted in a spherical coordinate system. $R[\mathrm{~m}]$ denote the radius of the sphere. . . . . . . . . . . . .

8-4 3D plot of the optimal spatial upper-limb movement for target 1. (Left) $3 \mathrm{D}$ view of the simulation, camera located at $\left(44.3530^{\circ}, 7.4481^{\circ}\right)$ in (azimuth-elevation coordinate) (Center) $x z$-plane view of the simulation (Right) $x y$-plane view of the simulation. The time-sequence of the movement is depicted, and the moment when the tip of the whip reached the target is emphasized. . . . . . . . . . . . .

8-5 3D plot of the optimal spatial upper-limb movement for target 2. (Left) $3 \mathrm{D}$ view of the simulation, camera located at $\left(44.3530^{\circ}, 7.4481^{\circ}\right)$ in (azimuth-elevation coordinate) (Center) $x z$-plane view of the simulation (Right) $x y$-plane view of the simulation. The time-sequence of the movement is depicted, and the moment when the tip of the whip reached the target is emphasized. . . . . . . . . . . . 
8-6 3D plot of the optimal spatial upper-limb movement for target 3. (Left) $3 \mathrm{D}$ view of the simulation, camera located at $\left(44.3530^{\circ}, 7.4481^{\circ}\right)$ in (azimuth-elevation coordinate) (Center) $x z$-plane view of the simulation (Right) D view of the simulation, camera located at $\left(-134.8516^{\circ}, 4.1426^{\circ}\right)$. The time-sequence of the movement is depicted, and the moment when the tip of the whip reached the target is emphasized. . . . . . . . . . 99

8-7 3D plot of the optimal upper-limb movement for target 1. Shoulder joint trajectory is colored in orange, and the end-point trajectory of the upper-limb is colored in blue. . . . . . . . . . . . . . . . . 100

8-8 3D plot of the optimal upper-limb movement for target 2. Shoulder joint trajectory is colored in orange, and the end-point trajectory of the upper-limb is colored in blue. . . . . . . . . . . . . . . . . 101

8-9 3D plot of the optimal upper-limb movement for target 3. Shoulder joint trajectory is colored in orange, and the end-point trajectory of the upper-limb is colored in blue. . . . . . . . . . . . . . 102

A-1 The $N$-node multi-pendulum model. The Cartesian coordinate frame with its origin $O$ is indicated at the top of the pendulum. . . . . . . 108

B-1 Diagram of possible paths of $x(t)$ from point $x\left(t_{i}\right)$ to point $x\left(t_{f}\right)$. Black line represents one of the possible paths which $x(t)$ could take, and red line denotes the deviated path from function $x(t)$ by function $\delta x(t)$. Note that all possible paths start from the same point, $x\left(t_{i}\right)$ and end at the same point, $x\left(t_{f}\right)$, i.e. $\delta x\left(t_{i}\right)=\delta x\left(t_{f}\right)=0 . \ldots \ldots$

B-2 Plot of position and velocity of minimum-jerk-trajectory $x(t)$ [Eqn. B.18]. A single peak unimodal bell-shaped speed profile of the minimumjerk-trajectory, with peak value $v_{\max }$, is shown in the right. Detailed value for $v_{\max }$ was $1.875 \cdot\left(x_{f}-x_{i}\right) / D$. . . . . . . . . . . .

C-1 Algorithm comparison: optimization with the short-whip model. Data from 400 to 600 iterations were plotted. . . . . . . . . . . 
C-2 Algorithm comparison: optimization with the medium-whip model. Data from 400 to 600 iterations were plotted. . . . . . . . . . 125

C-3 Algorithm comparison: optimization with the long-whip model. Data from 400 to 600 iterations were plotted. . . . . . . . . . . . . 125

C-4 Searching the optimal solution with the DIRECT algorithm in the 2dimensional search space. The optimal value for the function resided near point $\left(x_{1}, x_{2}\right)=(0.5,0.25)$. Image from [Finkel, 2003]. . . . 126 


\section{List of Tables}

3.1 Detailed parameters of the upper-limb model [Hatze, 1980]. Subscripts denote the shoulder and elbow joints, numbered proximal to distal. Principal moment of inertia of limb segments were calculated with respect to the center of mass (C.O.M.). A graphical description of the model is shown in [Figure 3-3]. . . . . . . . . . . . . . . . .

6.1 Detailed values of the experimentally-fitted whip model. $N[-]$ : number of nodes (i,e, sub-models) for the whip model; $m[k g]$ : the pointmass of each sub-model; $l[\mathrm{~m}]$ : length of the sub-model; $k[N \cdot \mathrm{m} / \mathrm{rad}]$ : coefficient of linear torsional spring of the rotational joint; $b[N \cdot m$. $s / \mathrm{rad}]$ : coefficient of linear torsional damping of the rotational joint. .

7.1 Detailed values of whip parameters for each whip model. . . . . . . .

7.2 Lower and upper bound of the 5 movement parameters used for the DIRECT-L optimization algorithm. . . . . . . . . . . . . 76

7.3 Optimal movement parameters and the corresponding output value $L^{*}$. 600 iterations were conducted with the DIRECT-L algorithm. . . . . 77

7.4 The mean, standard deviation (std.), maximum (Max), minimum (Min), coefficient of variation (C.O.V.) and standard error (S.E.) values of the 5 movement parameters which yielded $L^{*}$ values lower than $0.05 \mathrm{~m}$. The number of samples was $339 . \ldots \ldots \ldots$

7.5 Optimal movement parameters and the corresponding output value $L^{*}$. Exp.-fitted denotes experimentally-fitted whip model . . . . . . 86 
8.1 Lower bound (Low. B.) and upper bound (Up. B.) of the 9 movement parameters used for the DIRECT-L optimization algorithm. . . . . . 93

8.2 Optimal movement parameters and the corresponding output value $L^{*}$

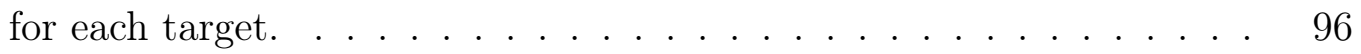

C.1 Minimum values of $L^{*}[m]$, within the 600 iterations. . . . . . . . 124 


\section{Chapter 1}

\section{Introduction}

Tool-use is a hallmark of human behavior. The dexterity required to handle a broad range of tools has been widely recognized as a distinctively human characteristic [Boesch and Boesch, 1990, Hunt, 1996, Johnson-Frey, 2004, Kenward et al., 2005, Lewis, 2006, Hogan and Sternad, 2012, Hogan, 2017]. Although many animals employ simple tools to extend their physical capabilities, there still is a significant gap between the performance of humans and non-human beings. The way humans make and use tools is perhaps what sets our species apart from any other animals.

Inspired by this salient feature, intensive research on human tool manipulation aims to eventually confer this dexterity onto robots. However, despite extensive research, the remarkable performance of humans has yet to be replicated, and a comprehensive theoretical framework remains to be established.

\subsection{The Paradox of Human Performance}

A paradox emerges when we recognize that humans vastly out-perform modern robot technology despite low-bandwidth actuators, imprecise sensors, high levels of noise and long latencies in neural communication within the neuromuscular system. The fastest neural transmission speed in humans is no more than $120 \mathrm{~m} / \mathrm{s}$ (animals' conduction speed is slower and less economical) [Kandel et al., 2000, Sperelakis, 2012]. Even the fastest neural transmission speed is a million times slower than in its robotic 
counterparts, which can conservatively reach up to $\sim 10^{8} \mathrm{~m} / \mathrm{s}$ [Myers, 2009].

Muscles, our biological actuators, are slow. The typical twitch contraction time, the time from an impulsive stimulus (e.g., electrical) by the nervous system to peak isometric tension, for the human biceps brachii is about 50ms [Kandel et al., 2000]. Assuming a linearized model to approximate this neuromuscular system results in a bandwidth of about $3 \mathrm{~Hz}$. In comparison, electromechanical technology routinely achieves bandwidth from tens to hundreds of Hz [Paine et al., 2013].

The brain, which is the central organ of the human nervous system, is also slow. The spatial cognitive performance of the brain was assessed from the classic "mental rotation" task [Shepard and Metzler, 1971]. It was reported that the average time required to determine whether two visual objects were the same in shape or mirror images (enantiomer), increased linearly with the angular difference in their orientation. Quantitatively, the average reaction time of the subjects required about 1 second plus 1 additional second per $60^{\circ}$, i.e. $\sim 4 \mathrm{~s}$ for $180^{\circ}$ rotation.

How can the sensorimotor capabilities of humans out-perform modern robots, despite their slow "hardware" (e.g., muscles) and "wetware" (e.g., neurons)?

\subsection{Internal Models in Human Motor Control}

Since the slow neuromuscular system impairs reactive control, the controller must rely heavily on prediction, as in model-predictive control (MPC) [Sakaguchi et al., 2015]. The considerable closed-loop delay of the neuromuscular system makes pure feedback control of large classes of fast movements impossible [Gerdes and Happee, 1994]. Even fast and coordinated arm movements, ubiquitous in everyday tasks, cannot be executed solely by feedback control [Kawato, 1999]. Thus, rather than achieving motor skills based on real-time intervention from the central nervous system (CNS), the slow neuromuscular system implies that prediction based on some form of internal model plays a prominent role in human motor control.

The concept of an internal model contributing to human motor control is not new. Over the last several decades, the hypothesis that the nervous system con- 
structs predictive internal models of the physical world has been a major focus in neuroscience [Wolpert et al., 1998, Kawato, 1999, Flanagan et al., 2003, Crevecoeur et al., 2010, Degallier and Ijspeert, 2010, McNamee and Wolpert, 2019]. However, although prediction based on some form of internal representation is widely accepted as a key aspect of human motor control, the nature of the internal model itself still remains unclear.

\subsection{Why study a Whip? - Complexity is Informative}

If an internal model plays a key role in human motor control, in what form will the internal model be? To address this problem, we focus on one of the most complex and exotic tools which humans can manipulate - a whip.

There are several reasons why studying the manipulation of an object as complex as a whip promises new insight. In particular, a whip forces us to confront the daunting complexity of tools which humans can routinely master. Previous motor neuroscience research has gained considerable insight from studying the Lagrangian dynamics of an open kinematic chain of rigid bodies. This explicit and/or implicit knowledge of body dynamics was postulated to be implemented in neural circuits involving the cerebellum, i.e. an "internal model" [Shidara et al., 1993, Wolpert et al., 1998, Kawato, 1999]. However, owing to the rapid growth of computational complexity with degrees-of-freedom, most of these studies were confined to models with a small number of degrees-of-freedom (often as few as two). Regarding motor learning as equivalent to some form of optimization process, real-time optimization of Lagrangian dynamic systems with as few as tens of degrees-of-freedom is profoundly challenging [Martínez-del Rincón et al., 2007, Khusainov et al., 2018]. Due to what Richard Bellman called the "curse of dimensionality", optimization suffers deeply from the exponential growth of computational complexity, and often fails to converge to an optimal solution [Bellman, 2015].

A whip has vastly more degrees-of-freedom with significant dynamics. A whip is a flexible object with non-uniform mechanical properties that interacts with a 
compressible gas up to the supersonic regime. A competent engineering-style model to describe the whip dynamics requires nonlinear partial differential equations of infinite order [Bernstein et al., 1958, Goriely and McMillen, 2002, McMillen and Goriely, 2003]. This complexity is not confined to whips: similar dynamics are observed in common objects such as tablecloths and towels. The daunting complexity innate to these extremely high degree-of-freedom (DOF) objects (in principle an infinite number), is almost unmanageable, taxing even modern super-computers.

Nevertheless, apparently indifferent to this daunting complexity, humans can learn to manipulate a whip, sometimes with apparent ease, with some "whip masters" reaching an impressive level of expertise [Henrot, 2016]. In preliminary studies we found that an expert whip master could wield a 6 -foot $(\sim 1.8 \mathrm{~m})$ bullwhip to hit a 2.5 -inch $(\sim 6.4 \mathrm{~cm})$ diameter target at a distance of 6 -foot $(\sim 1.8 \mathrm{~m})$ with a success rate of more than $90 \%$. This observation suggests that the CNS employs a profoundly different approach than optimization based on an engineering-style model.

\subsection{Dynamic Motor Primitives Hypothesis}

Mounting evidence indicates that human sensorimotor control relies on a composition of primitive dynamic actions [Sternad et al., 2000, Thoroughman and Shadmehr, 2000, Flash and Hochner, 2005, Kargo and Giszter, 2008, Sternad, 2008, Sing et al., 2009, Degallier and Ijspeert, 2010, Dominici et al., 2011]. Instead of containing an exact model of the tool being manipulated, we propose that humans simplify the motor task by composing motor control using "dynamic motor primitives" [Hogan and Sternad, 2012, Hogan and Sternad, 2013, Hogan, 2017].

"Dynamic motor primitives" refers to dynamic behaviors that manifest as stable attractors of the (nonlinear) neuromechanical system. They are conceived as dynamic "building blocks" that may be combined to produce complex behavior. Three classes of dynamic primitives have been identified - submovements, oscillations and mechanical impedances, the latter to manage physical interaction - though there may be others [Hogan and Sternad, 2012, Hogan, 2017] [Figure 1-1]. We hypothesize that the 
internal model used for (at least some) cognitive operations (e.g., learning, recalling, predicting and planning) is based on dynamic motor primitives.

\section{Dynamic Motor Primitives}



\#Discrete \#Point-to-Point

\section{Oscillations}

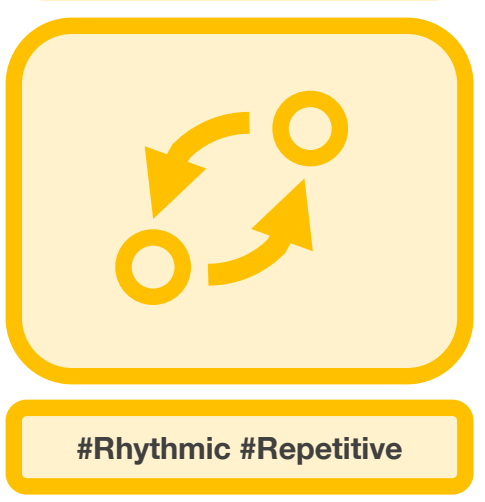

Mechanical Impedances

$$
Z: \Delta x(t) \rightarrow \boldsymbol{f}(t)
$$

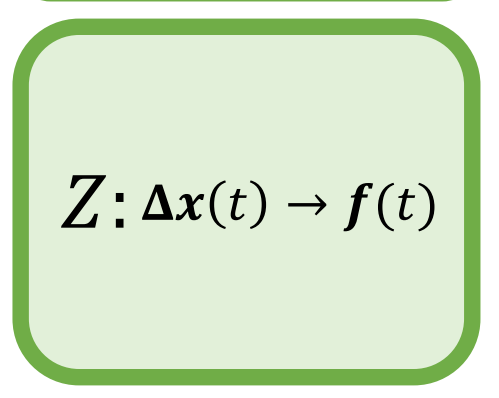

\#Interaction \#Operator

Figure 1-1: Three dynamic motor primitives - submovements, oscillations and mechanical impedances.

To test this hypothesis, we studied how humans learn to manipulate a complex object with significant dynamics - a whip. Encoding motor tasks via parameterized dynamic primitives may dramatically simplify the control of complex objects, thus enabling dexterous manipulation with minimal high-level supervision and intervention. Using optimization as a model of learning, this parameterization may enable convergence (or accelerate it) without encountering limitations due to the "curse of dimensionality".

The thesis set out to test (in simulation) whether a target could be reached with a whip using a (small) number of dynamic primitives, whose parameters were learned through optimization. We found that this approach was able to identify an upperlimb movement which could approximate a distant target with a whip. This result supports our hypothesis that composing actions using dynamic motor primitives may be a strategy which humans use to enable their remarkable dexterity. A similar approach may contribute to improved robot control. 


\subsection{Overview of Thesis}

This research aims to validate the following hypothesis: humans simplify motor tasks by employing an internal model based on dynamic motor primitives. This introductory chapter provided the reasoning that led to this hypothesis, and emphasized the importance of this study.

Chapter 2 delves into the basic definition of each dynamic motor primitive in detail — submovements, oscillations and mechanical impedances. Using mathematical language, definitions for each primitive are presented, and the Norton equivalent network model, which combines these primitives, is discussed.

Chapter 3 introduces the upper-limb and whip model used for simulations. The models differ depending on the dimensionality of the movements, i.e. whether movement is in $2 \mathrm{D}$ or $3 \mathrm{D}$ space.

Chapter 4 discusses the numerical accuracy of the MuJoCo (Multi-Joint dynam-

ics with Contact) software used for simulation [Todorov et al., 2012]. How well the numerical integrator used for the simulation complies with the law of energy conservation was tested.

Chapter 5 discusses a theoretical analysis of a linearized 2D planar whip model. Eigenvalue and eigenvector analysis of the linearized whip model was conducted.

Chapter 6 discusses experimental estimation of the parameters of the whip model. By conducting a simple experiment with the actual whip, the model parameters of the 2D planar whip model were identified.

Chapter 7 discusses a 2D planar whip task. With the planar model described in [Chapter 3], an upper-limb controller and a whip task to be optimized are defined. Detailed results of the optimization are presented.

Chapter 8 discusses a 3D spatial whip task. The whip task defined in [Chapter 7] is generalized to $3 \mathrm{D}$, and optimization results of the spatial whip task are presented.

Finally, chapter 9 presents conclusions of the present work, the relation to prior research, limitations of the research and discussion of further inquiry which should be addressed in the near future. 


\section{Chapter 2}

\section{Dynamic Motor Primitives}

Dynamic motor primitives are defined as patterns of behavior that emerge from dynamic systems, that is, as attractors [Hogan and Sternad, 2013]. An attractor is a subset of state-space with (at least) two main properties. First, it is an invariant set: if the system resides in an invariant set, it never leaves. ${ }^{1}$ Second, the invariant set is attractive: if the initial condition of the system starts sufficiently close to the invariant set, the system will eventually converge to the attractor.

Three distinct classes of dynamic motor primitive are proposed as a foundation for a comprehensive theoretical framework - submovements, oscillations and mechanical impedances. Submovements and oscillations provide a basis for unconstrained movements, and mechanical impedances account for contact and physical interaction with objects. For combining the three dynamic motor primitives, a Norton equivalent network model is re-purposed and extended to provide a unified framework.

\section{$2.1 \quad$ Submovements}

One class of dynamic primitives manifests as submovements. A submovement is conceived as a coordinative atom: just as atoms are primitive units of chemical reactions,

\footnotetext{
${ }^{1} \mathrm{~A}$ simple example is the eigenspace corresponding to a specific eigenvalue of a linear operator. Let the eigenvalue and eigenvector of linear operator $\boldsymbol{A}$ be $\lambda$ and $\boldsymbol{v}$, respectively. Then the $W=\operatorname{span}\{\boldsymbol{v}\}$ is an invariant subspace of dimension 1 with respect to operator $\boldsymbol{A}$, and the elements of subspace $W$ are $\boldsymbol{A}$ invariant.
} 
submovements are elements of dynamic coordination for composing motor behavior [Hogan and Sternad, 2012].

To render precision with mathematical language, let $\boldsymbol{x}$ be a $n$-length vector, $\boldsymbol{x} \in$ $\mathbb{R}^{n}$, which contains coordinate information (position, orientation etc.) with respect to a specific coordinate frame. We define a submovement as an attractor that describes a smooth transition of a variable from one value to another with a stereotyped time profile. In particular, a single submovement is defined as a stereotyped unimodal speed profile with basis function $\sigma(t)$ :

$$
\dot{x}_{j}(t)=\hat{v}_{j} \sigma(t), \quad j=1,2, \cdots, n
$$

where $x_{j}$ is the $j^{t h}$ variable of vector $\boldsymbol{x} ; \sigma(t)$ is the unit basis function with a unimodal profile; $\hat{v}_{j}$ is the speed amplitude which scales the unit basis function $\sigma(t)$; dots denote derivatives with respect to time.

The basis function $\sigma(t)$ has a non-zero positive value within a single finite duration, and the function value is zero outside of that domain. The derivative of function $\sigma(t)$ is zero at the peak, and the peak value is 1, i.e. if the time where the peak happens is taken to be $t_{p}$, then $\dot{\sigma}\left(t_{p}\right)=0$ and $\sigma\left(t_{p}\right)=1$ [Figure 2-1].

These speed profiles with different amplitude and duration can be superimposed along time. Assuming that $m$ submovements with the same speed profile $\sigma(t)$ are combined, the resulting speed profile of variable $x_{j}$ is:

$$
\dot{x}_{j}(t)=\sum_{k=1}^{m} \hat{v}_{j k} \sigma\left(t \mid b_{k}, d_{k}\right), \quad j=1,2, \cdots, n
$$

where $\hat{v}_{j k}$ is the speed amplitude of the $j^{\text {th }}$ coordinate of the $k^{\text {th }}$ submovements; $b_{k}$ and $d_{k}$ is the start time and duration of the $k^{t h}$ submovement, respectively [Figure $2-2]$. 


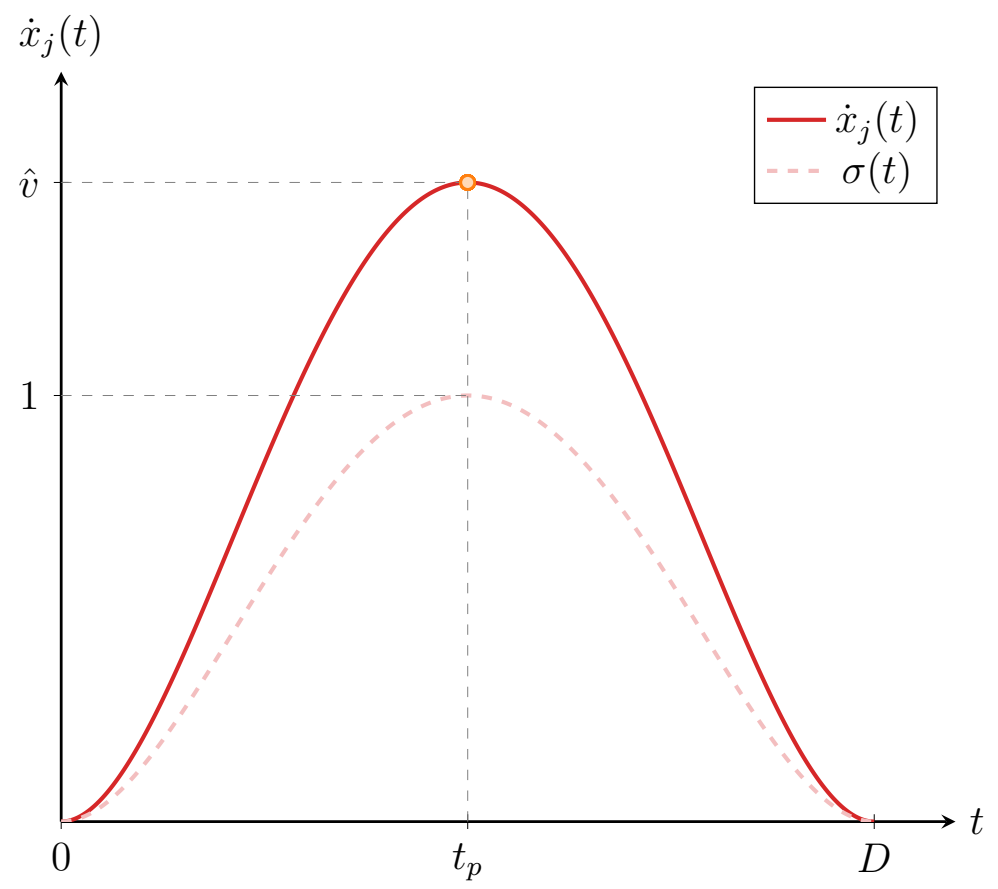

Figure 2-1: Plot of a representative single submovement with a unimodal speed profile $\dot{x}_{j}(t)$. The basis function $\sigma(t)$ is plotted as a dashed line. $\hat{v}$ is the peak speed of the submovement; $D$ is the duration of the submovement; $t_{p}$ is the time when the peak happened.

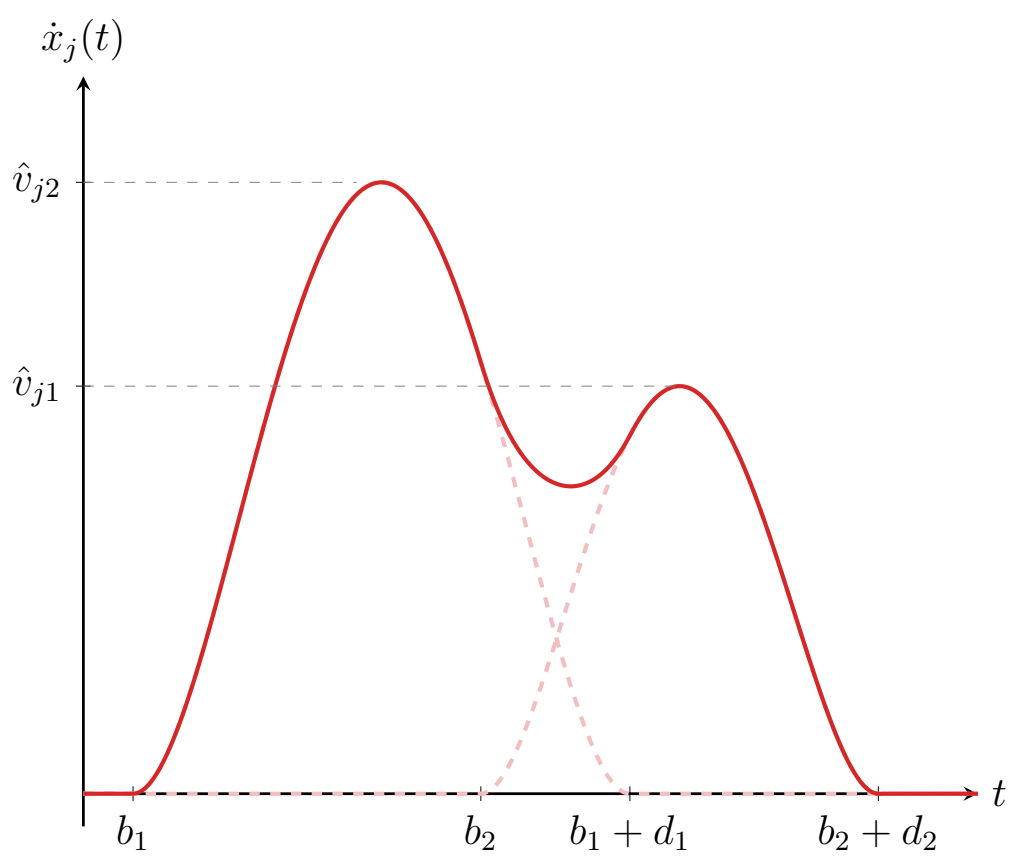

Figure 2-2: Plot of 2 superimposed submovements. Individual submovements are plotted as dashed lines. $b, d$ and $\hat{v}_{j}$ are the start time, duration and speed amplitude of $j^{\text {th }}$ coordinate for each submovement. 
The way the movements are composed makes the major difference between submovements and discrete movements. Submovements are like discrete (rest-to-rest) movements but they may overlap in time and their profiles may superimpose [Hogan and Sternad, 2013]. Claiming that any consistent definition of discrete movements requires that it should begin and end with a period of no movement, discrete movements can only be "sequenced" and cannot be "overlapped" in time [Hogan and Sternad, 2007, Hogan and Sternad, 2013]. Considering the rich variety of movements which humans exhibit, composing movements solely of discrete movements would severely restrict the repertoire of movements which can be generated. Defining submovements which can overlap in time can overcome this limitation and serve as competent "building blocks" of observable motor behavior.

Submovements may require complex patterns of neuromuscular activity to generate movement. However, the definition of submovements is deliberately confined to the behavioral or observational level, remaining silent about possible generative dynamic processes that may give rise to these observations [Hogan and Sternad, 2012].

\subsection{Oscillations}

From a strictly mathematical perspective, rhythmic movements may not be primitive. In principle, overlapping submovements are sufficient to describe all observed movements, since oscillatory or repetitive motor behaviors can be decomposed to a finite number of submovements. However, biological observations have shown that repetitive and discrete movements may be distinguished neurally [Brown, 1911, Brown, 1914, Grillner and Wallen, 1985, Schaal et al., 2004, Hogan and Sternad, 2012]. Rhythmic movement is very old phylogenetically and available evidence indicates that oscillatory behavior is a distinct dynamic primitive of biological motor control (and not a composite of submovements).

Neuroscientific evidence showed that rhythmic arm movement cannot be a composite of discrete movements [Schaal et al., 2004]. For a simple discrete/repetitive wrist joint movement, functional magnetic resonance imaging (fMRI) showed that 
discrete and rhythmic movements involved distinct regions of the brain. In detail, discrete movement involved several higher cortical planning areas in addition to cortical areas activated in rhythmic movement, even when both movements were performed by the same single wrist joint. This result showed that rhythmic movements cannot be a composite of discrete movements, although the possibility that discrete movements are truncated rhythmic movements remains open.

For the numerous variations and degrees of periodicity shown in rhythmic and repetitive movements, we denote these classes of dynamic primitive as "oscillations". In mathematical formulation, an oscillation primitive is defined as an attractor that describes an almost-periodic motion. Let $\boldsymbol{x}$ be an $n$-length vector, $\boldsymbol{x} \in \mathbb{R}^{n}$, which contains the coordinate information (position, orientation etc.) with respect to a specific coordinate frame. A mathematical condition for motion to be almost-periodic can be defined as:

$$
\left|x_{j}(t)-x_{j}(t+\Delta t+l T)\right|<\epsilon_{j}
$$

where $x_{j}$ is the $j^{\text {th }}$ variable of $\boldsymbol{x} ; T$ is a constant; its smallest value is the period of the movement; $l$ is an integer; $|\Delta t|<\delta$ is a small deviation of each cycle duration from the period; $\epsilon_{j}$ and $\delta$ are small constants. As with submovements, this definition of an oscillation is silent about the physiological-level mechanism which gives rise to the observed behavior. The definition is deliberately confined to the behavioral or observational level of analysis.

\subsection{Mechanical Impedances}

As submovements and oscillations provide a basis for unconstrained movements, they may be sufficient to describe the observed unconstrained motor behavior of humans. To account for contact and physical interaction with the environment, a third class of dynamic primitives is required, mechanical impedances.

The term impedance was first coined by Oliver Heaviside in the study of electrical circuits [Heaviside, 2011]. In the electrical domain, the impedance of an element is defined as a functional relation between the input current through the element (flow 
variable), and the output voltage across the element (effort variable). However, the definition of impedance need not be confined to the electrical domain. If the power conjugate relationship of flow and effort is consistent, i.e. the inner product between flow and effort variable yields power, the definition of impedance can be generalized to multiple domains. For instance, in the domain of mechanical translation, velocity (flow) and force (effort) define a power conjugate pair, and in the domain of mechanical rotation, angular velocity (flow) and torque (effort) define a the power conjugate pair [Borutzky, 2011]. To expand the definition of impedance to the mechanical domain, the mechanical translation pair is used for convenience.

The effort variable for mechanical translation is force $\boldsymbol{f}$, and the flow variable is velocity $\boldsymbol{v}$. However, configuration (or position) plays a special role in mechanical systems that is not encountered in electrical circuits. Restricting inputs to velocities (analogous to electrical currents) would not take this distinction into account. The essential concept of (mechanical) impedance is an operator that yields an output exertion variable (force or any of its integrals or derivatives) in response to an input motion variable (velocity or any of its integrals or derivatives). To account for the special role of configuration in mechanical system, mechanical impedance is defined as a dynamic operator that determines the force $\boldsymbol{f}(t)$ (time-history), evoked by an imposed displacement $\Delta \boldsymbol{x}(t)$ (time-history).

$$
Z: \Delta \boldsymbol{x}(t) \rightarrow \boldsymbol{f}(t)
$$

The imposed displacement $\Delta \boldsymbol{x}(t)$ is defined as the deviation of the actual trajectory $\boldsymbol{x}(t)$, from the "zero-force trajectory" $\boldsymbol{x}_{0}(t)$ of the mechanical system, i.e. $\boldsymbol{\Delta} \boldsymbol{x}(t)=$ $\boldsymbol{x}_{0}(t)-\boldsymbol{x}(t)$. Note that the form of the impedance operator $Z\{\cdot\}$ may be nonlinear and time-varying. For brevity, the "mechanical" prefix is often omitted.

To define the imposed displacement $\Delta \boldsymbol{x}(t)$, a construct called zero-force trajectory is defined. The zero-force trajectory is the trajectory that would have been followed if the external forces were zero. ${ }^{2}$ Mathematically, in the absence of external forces

\footnotetext{
${ }^{2}$ The term zero-force trajectory is mainly used throughout this paper. However if the motion is planned in the joint-space coordinate, the term zero-torque trajectory is used instead.
} 
or constraints, no force is exerted when the actual trajectory, $\boldsymbol{x}(t)$ exactly matches with the zero-force trajectory, $\boldsymbol{x}_{0}(t)$. The zero-force trajectory summarizes one consequence of neural activity and expresses it as a quantity that may be compared with actual motion [Hogan and Sternad, 2013, Hermus et al., 2020]. This concept of zeroforce trajectory allows us to define the displacement input to the impedance operator $Z\{\cdot\}$, which generates the corresponding force output.

As long as the impedance operator is invertible, given measured actual position $\boldsymbol{x}(t)$ and force $\boldsymbol{f}(t)$, the zero-force trajectory can always be identified at any time [Hermus et al., 2020]. The mere existence of the zero-force trajectory $\boldsymbol{x}_{0}$, under the assumption that the inverse of the impedance operator exists, is not by itself very surprising. Therefore, the existence of the zero-force trajectory does not per se provide compelling evidence about how the CNS actually encodes motor commands to generate observable behavior. The definition of zero-force trajectory can be used to describe observable neuromechanical behavior, yet may have no relation to how the CNS actually gives rise to this behavior.

It may be questioned how to incorporate impedance, which is a mathematical mapping or operator from one variable to another, into the definition of attractive invariant sets. However, the set of objects need not be limited to a set of state variables. Just as a set of matrices or a set of operators can be defined, it is possible to define an "operator space" consisting of mathematical operators or mappings.

Compared to submovements and oscillations which define how motion may be composed and structured, mechanical impedance regulates physical interaction between a human and its environment. By dynamically regulating the relation between displacement and force, mechanical impedance determines how the system interacts with a variety of environments.

Neuromechanical impedance arises in part from intrinsic properties of muscles, and humans can voluntarily modulate the mechanical impedance of their limbs. One way to modulate impedance is by co-activation of agonist-antagonist muscle groups. Opposing muscle groups activated simultaneously generate net torque via the difference of their actions about a joint. However, mammalian muscle mechanical impedance 
increases with generated muscle force. The sum of muscle activity determines the net mechanical impedance about the joint, even if the net generated torque is unchanged. Thus, at the same static posture, humans can exhibit different responses to external disturbance via impedance modulation.

Mechanical impedance is also influenced by feedback loops at the spinal level or higher [Nichols and Houk, 1976, Hoffer and Andreassen, 1981]. It was reported that the gains of these feedback loops are highly modifiable, and one of the major means is via gamma motor neuron activity [Prochazka et al., 2000]. Roughly speaking, gamma motor neurons adjust the sensitivity of muscle spindles (which are stretch receptors within the body of a muscle that detect changes in the length of the muscle), thereby regulating the resulting muscle forces. The larger the gain of the gamma neuron is, the greater the change in generated muscle force that results from a given amount of stretch applied to the (intrafusal fibers of the) muscle [Purves et al., 2004].

\subsection{Norton Equivalent Network Model}

How can we combine these dynamic primitive elements, and build up an internal model that can competently represent the human motor behavior?

The first step is to recognize the two different domains in which the dynamic primitives are situated: the information processing domain and the energy processing domain. Submovements and oscillations belong to the information processing domain: these primitives plan the motion of the system with far fewer constraints than elements

in the energy processing domain. The only constraints on the information processing domain are temporal causality, i.e. no output before the corresponding input, and boundedness of the information signal.

Mechanical impedances, which manage physical interaction with the environment, belong to the energy processing domain: physical interaction with the environment is by nature bi-lateral — each system affects the other. On top of the constraints of temporal causality and boundedness, they are additionally subject to physical constraints such as energy conservation, entropy production, etc. 
One versatile structure for combining the two different domains of motion primitives (i.e. submovements, oscillations) and interactive primitives (i.e. impedances) is a nonlinear extension of the classical electrical equivalent circuit [Hogan, 2013, Hogan, 2017]. The port behavior of a complex electrical circuit with an arbitrary number of interconnected sources and linear circuit elements may be exactly modeled by an equivalent source and an equivalent impedance or admittance. An analogous structure may be defined for a broad class of interconnected nonlinear elements. Depending on the type of equivalent source (motion or force), and the configuration of the corresponding equivalent impedance (parallel, common force connection or series, common motion connection), the result is a Norton or Thèvenin equivalent network model.

Between the two types of model, the Norton equivalent network model provides a competent structure for how the three classes of dynamic primitives may be related and combined [Hogan, 2013]. The Norton equivalent network is chosen since the representation preserves the property of "translational invariance": even though the reference frame origin is translated, the resulting interactive behavior does not change. This property of "translation invariance" is a desirable geometric symmetry for representing interactive dynamics, and this property is well preserved via an equivalent motion source and common force connection of a Norton model rather than an equivalent force source and common motion connection of the Thèvenin model. Modeling the net interactive behavior of human motor dynamics with a Norton network model offers us a unified description of how central commands from the CNS and peripheral mechanics cooperate to produce observable behavior.

A diagram of a Norton equivalent network model is shown in [Figure 2-3]. Two commands from the CNS completely determine the whole interactive dynamics: a motion command via the equivalent motion source and an impedance command which regulates the equivalent impedance. The CNS sends a uni-directional feed-forward command to the motion source. The forward-path dynamics of the equivalent motion source result in an output which can be summarized as a zero-force trajectory $\boldsymbol{x}_{0}$. With a given actual trajectory $\boldsymbol{x}$, displacement $\Delta \boldsymbol{x}$ is determined. Impedance $Z\{\cdot\}$ specified by the CNS, determines the output force $\boldsymbol{f}$ from the input displacement $\Delta \boldsymbol{x}$ 
evoked by the interaction. Summarizing, the output of the uni-directional forwardpath dynamics, which consists of submovements and oscillations, combined with the bi-directional interactive dynamics, characterized by (equivalent) impedance, specifies the dynamics of the whole model.



Figure 2-3: Diagram of a Norton equivalent network model of the human motor control system. A nonlinear equivalent network relating motion planning in the information domain with interactive dynamics in the energy domain [Hogan, 2013].

There are two key benefits of representing the neuromuscular behavior of humans by a Norton equivalent network model. One is prodigious simplification: the biological system of humans is excruciatingly complex and largely uncharted [Kandel et al., 2000]. Considering this exceedingly complex structure, our ability to study the internal details of this "black box" are quite limited. Nonetheless, based on the Norton equivalent network formulation, the behavior at an interaction port of an arbitrarily complex system can be completely summarized in a simpler form which is functionally equivalent. The other benefit is that the elements comprising the network model are experimentally identifiable (at least in principle) [Hodgson and Hogan, 2000, Hermus et al., 2020]. Based on this simplified model, the two parts of the model, the equivalent motion source and equivalent impedance, can be identified from experiments in situ. The resulting summary representation with experimentally identifiable parts can significantly facilitate analysis. 


\section{Chapter 3}

\section{Modeling}

The research presented in this paper used the simulation software called MuJoCo (Multi-Joint dynamics with Contact) [Todorov et al., 2012]. Models for conducting whip manipulation tasks in the simulation environment were developed to test the dynamic motor primitives hypothesis.

The models used for the simulation consisted of two main parts: an $N$-node model of a whip (the object being manipulated) and a model of the human upper limb (the manipulator). Various types of each model were constructed, and various combinations of whip and upper-limb models were studied.

\subsection{Whip Model Type 1 - Planar}

An $N$-node "lumped-parameter" model was developed to approximate the continuum dynamics of a whip. It consisted of a finite sequence of serially-connected planar sub-models. Each sub-model was composed of three lumped-parameter elements: an (ideal) point mass, a linear torsional spring and a linear torsional damper. The pointmass $m[k g]$ was suspended from a single degree-of-freedom pivot with length $l[m]$. The pivot, a rotational joint, was equipped with a linear torsional spring and a linear torsional damper, with coefficients $k[N \cdot \mathrm{m} / \mathrm{rad}]$ and $b[N \cdot \mathrm{m} \cdot \mathrm{s} / \mathrm{rad}]$, respectively. $N$ of these identical planar sub-models were serially connected to comprise the $N$-node whip model. The parameters $(N, l, m, k, b)$ were called the "whip parameters" of 
the model [Figure 3-1].
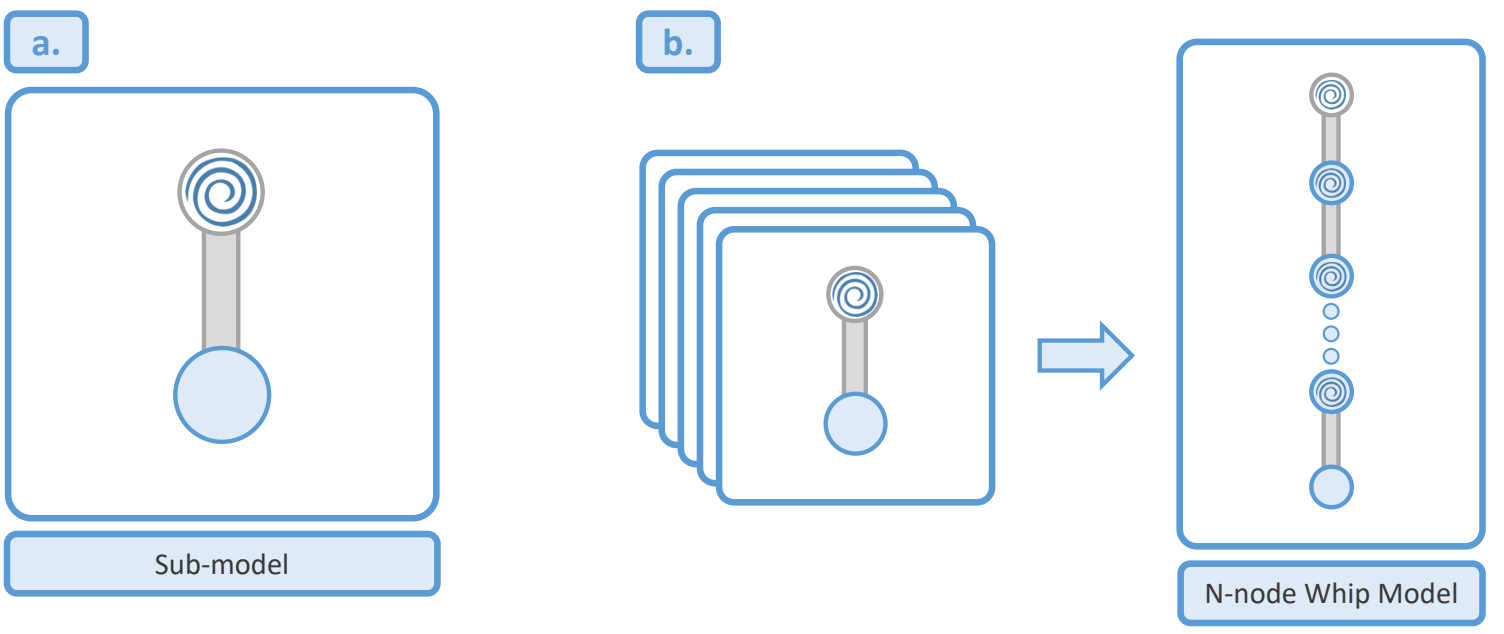

Figure 3-1: (a) Planar sub-model of the $N$-node whip model. The blue filled circle depicts the (ideal) point mass $m$ of the sub-model. The mass $m$ was attached to the grey massless cylinder with length $l$. The swirl icon on the pivot symbolizes the linear torsional spring $k$ and linear torsional damper $b$. (b) $N$ identical sub-models comprised the $N$-node whip model.

\section{$3.2 \quad$ Whip Model Type 2 - Spatial}

A 3D spatial whip model was developed to reproduce the 3D movement of the whip. On top of the 2D sub-model presented in [Section 3.1], an additional single degree-offreedom pivot was added perpendicular to the original pivot [Figure 3-2]. Assuming that the stiffness and damping properties of the whip were isotropic (i.e. the physical property of the whip was the same when measured in different directions), the same linear torsional spring $k$ and linear torsional damper $b$ were assigned to the additional pivot. Summarizing, each node of the whip consisted of two rotational joints, where the axes were perpendicular to each other. 


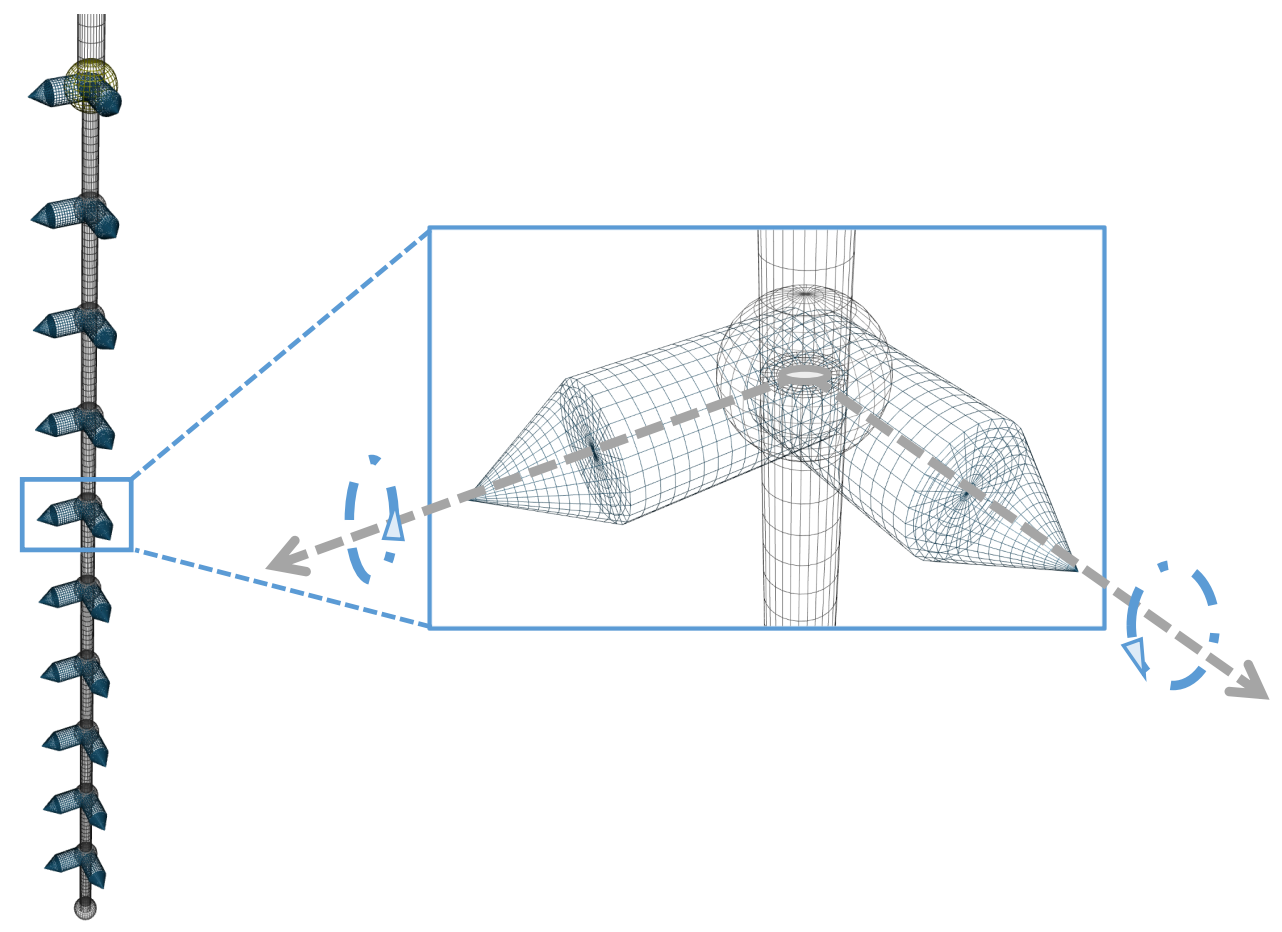

Figure 3-2: A 3D spatial model of the $N$-node whip model. Axes of rotation are visualized as a bullet shape. Each node of the whip had 2 degrees-of-freedom. The 3D whip model was modeled and rendered with the MuJoCo simulator.

\subsection{Upper-limb Model Type 1 - Planar}

The human upper-limb was modeled as a two-bar open-chain linkage. The fingers, hand and wrist (everything distal to the wrist joint) were excluded from this model. The flexion/extension movement of the shoulder and elbow were modeled as single degree-of-freedom rotational joints. The shoulder joint axis was fixed in space, and the movement of the upper-limb model was confined to the sagittal plane. Independently controlled torque actuators were mounted co-axially with the shoulder and elbow joints. The two limb segments were taken to be non-uniform cylinders, i.e. the center of mass (C.O.M.) and the geometric center of the limb segment were not identical. The geometrical and inertial parameters for each limb segment were obtained from a computational model by Hatze [Hatze, 1980], and detailed values used for the model are presented in [Table 3.1]. 

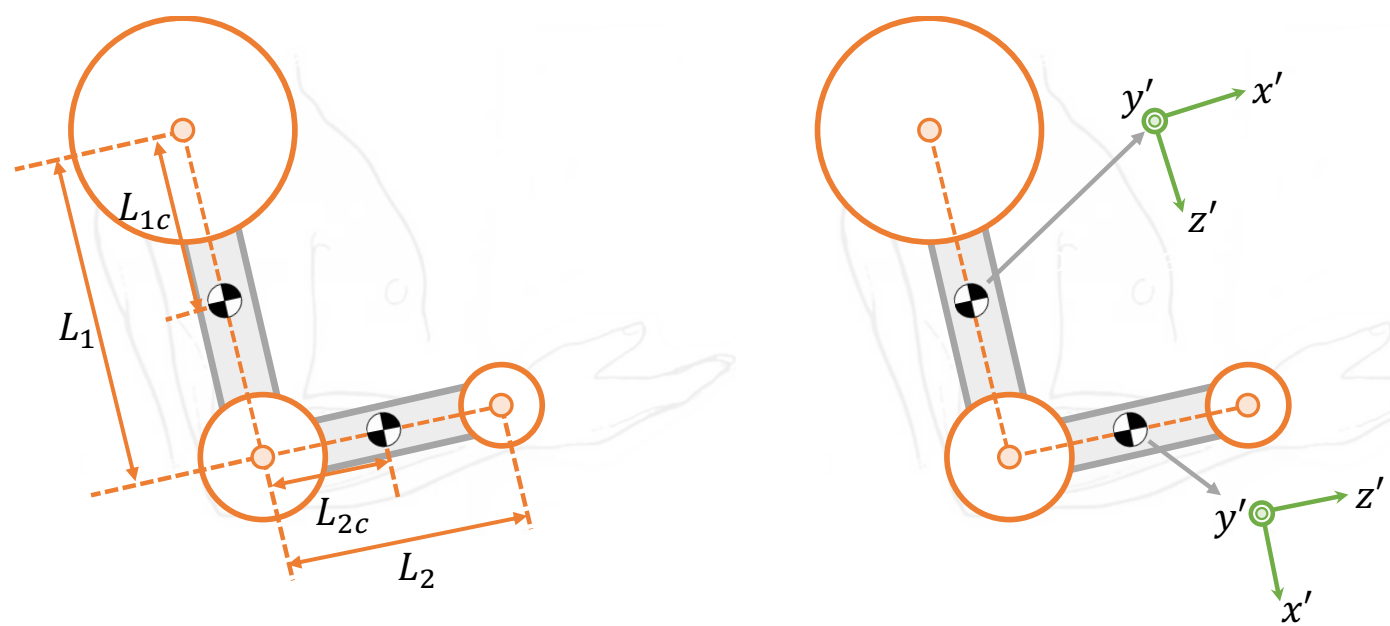

Figure 3-3: The two-bar planar upper-limb model. $x^{\prime}, y^{\prime}$ and $z^{\prime}$ denote the axes of the principal moments of inertia for each limb segment. Values and description of the mathematical notation are shown in [Table 3.1].

\begin{tabular}{llccr}
\hline \hline & Description & Notation & Values & Unit \\
\hline \multirow{2}{*}{ Limb Inertia } & Mass of Limb Segment & $M_{1}, M_{2}$ & $1.595,0.869$ & {$[\mathrm{~kg}]$} \\
& Length of Limb Segment & $L_{1}, L_{2}$ & $0.294,0.291$ & {$[\mathrm{~m}]$} \\
& Length from proximal joint to C.O.M. & $L_{1 c}, L_{2 c}$ & $0.129,0.112$ & {$[\mathrm{~m}]$} \\
& Principal Moment of Inertia, x'-axis & $I_{1, x x}, I_{2, x x}$ & $0.0119,0.0048$ & {$\left[\mathrm{~kg} \cdot \mathrm{m}^{2}\right]$} \\
& Principal Moment of Inertia, y'-axis & $I_{1, y y}, I_{2, y y}$ & $0.0119,0.0049$ & {$\left[\mathrm{~kg} \cdot \mathrm{m}^{2}\right]$} \\
& Principal Moment of Inertia, z'-axis & $I_{1, z z}, I_{2, z z}$ & $0.0013,0.00047$ & {$\left[\mathrm{~kg} \cdot \mathrm{m}^{2}\right]$} \\
\hline
\end{tabular}

Table 3.1: Detailed parameters of the upper-limb model [Hatze, 1980]. Subscripts denote the shoulder and elbow joints, numbered proximal to distal. Principal moment of inertia of limb segments were calculated with respect to the center of mass (C.O.M.). A graphical description of the model is shown in [Figure 3-3].

\subsection{Upper-limb Model Type 2 - Spatial}

A spatial 4 degrees-of-freedom upper-limb model was developed to reproduce 3D upper-limb movements. Based on the 2 degrees-of-freedom planar upper-limb model in [Section 3.3], two additional rotational joints perpendicular to each other were added to the shoulder joint, i.e. adduction/abduction movement (J2), lateral/medial rotation movement (J3) were added to the flexion/extension movement (J1) [Figure 
3-4]. For all of the four joints of the upper-limb model, independently controlled torque actuators were mounted co-axially. The geometrical and inertial parameters for each limb segment were identical to the upper-limb model type 1 [Section 3.3], which are shown in [Table 3.1].



Figure 3-4: A spatial upper-limb model rendered with the MuJoCo simulator. A Cartesian coordinate frame is indicated at the shoulder joint. Joint J1, J2 and J3 were perpendicularly connected to reproduce a 3D shoulder joint movement. The order of rotations of the three shoulder joints were ordered with ascending number, i.e. J1 J2 - J3. J1, J2 and J3 corresponded to flexion/extension, adduction/abduction and lateral/medial rotation, respectively. Axes of rotation are visualized as a bullet shape. 


\section{Chapter 4}

\section{Validation of the MuJoCo simulator}

To assess the reliability of the MuJoCo simulator, how well the numerical integrator used for the simulation complied with the law of energy conservation was tested. The law of energy conservation was used as a measure of simulation accuracy.

The total (kinetic + potential) energy of a lossless whip model was monitored as a function of time. A planar $N$-node whip model [Section 3.1] was used for the simulation. The lossless model was created by setting the torsional damping coefficient $b$ to zero for the $N$ sub-models. For all of the MuJoCo simulations, the semi-implicit Euler method was chosen as the numerical integrator, with a time step of $0.1 \mathrm{~ms}$.

Two separate methods were conducted for the validation: an initial condition response method [Section 4.1.1] and a displacement function excitation method [Section 4.1.2]. Both methods monitored the total energy of the whole lossless whip model with respect to time. At each time step, the potential and kinetic energy of the whole system were called by printing out the Energy data array under mjData.

\subsection{Methods}

\subsubsection{Method 1 - Initial Condition Response}

The simulation consisted of a lossless $N$-node whip model suspended from a freelyrotating pivot fixed in space. No external actuators or external energy sources were 
included in the simulation, i.e. the whip model was a closed system, where no energy was exchanged with the surroundings.

The simulation started by setting free the lossless whip model, which was at rest in a specific initial position [Figure 4-1]. As the whip model swung to and fro under the influence of gravity, the total energy of the lossless whip model was monitored. Various initial positions of various $N$-node whip models were tested. The data were extracted with a sampling rate of $50 \mathrm{~Hz}$, and the simulation was conducted for 60 seconds.

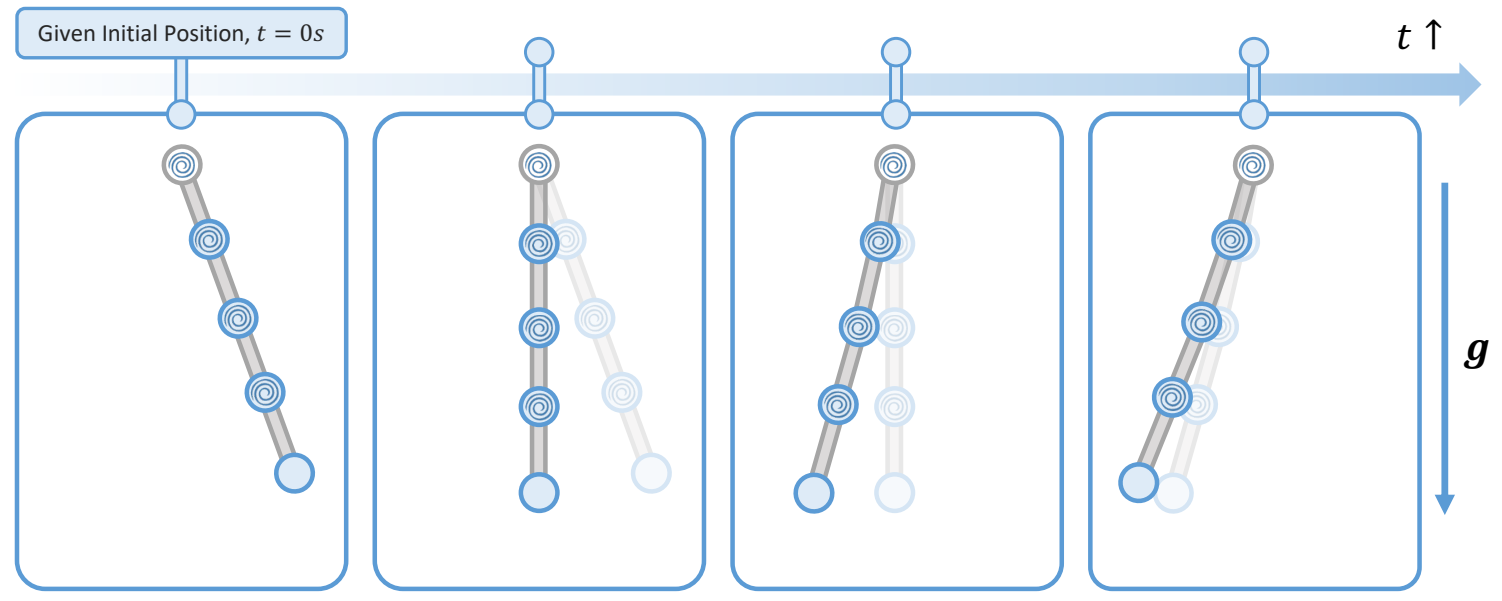

Figure 4-1: The initial condition response method. From a given initial position at time $t=0 \mathrm{~s}$, the lossless whip model was set loose and the energy of the system was monitored. Vector $\boldsymbol{g}$ denotes gravity of the simulation environment.

\subsubsection{Method 2-Displacement Function Excitation}

The simulation started with the lossless whip model in an equilibrium configuration, at rest hanging vertically downward. A single degree-of-freedom linear actuator, driven by a position controller with (extremely) high proportional gain, moved the top of the modeled whip in a horizontal motion from rest with a position input described by function $p(t)$ :

$$
p(t)=\frac{A}{2}\left\{1-\cos \left(\frac{2 \pi}{w} \cdot\left(t-t_{0}\right)\right)\right\}
$$


where $A[m]$ is the amplitude of the motion profile, $t_{0}[s]$ is the time when the motion started and $w[s]$ is the duration of the motion; $t[s]$ is the time variable defined in the domain $\left[t_{0}, t_{0}+w\right]$. For times before and after this interval, the position controller remained at rest, i.e. $p(t)=0[m]$ [Figure 4-2]. The simulation was run for 5 minutes, and the value of total energy was extracted with a sampling rate of $50 \mathrm{~Hz}$.


Figure 4-2: The displacement function excitation method. The upper graph depicts the representative function profile of displacement function $p(t)$ [Eqn. 4.1]. $t_{0}[s]$ denotes time when the displacement function started and $w[s]$ denotes the duration of the motion. From the equilibrium configuration at rest hanging vertically downward, the whip was excited with function $p(t)$ from time domain $\left[t_{0}, t_{0}+w\right]$. 


\subsection{Results}

\subsubsection{Method 1 - Initial Condition Response}

A single degree-of-freedom 1-node whip model (single pendulum) was first tested. The 1-node whip model was positioned just at the vicinity of the upward unstable equilibrium posture.

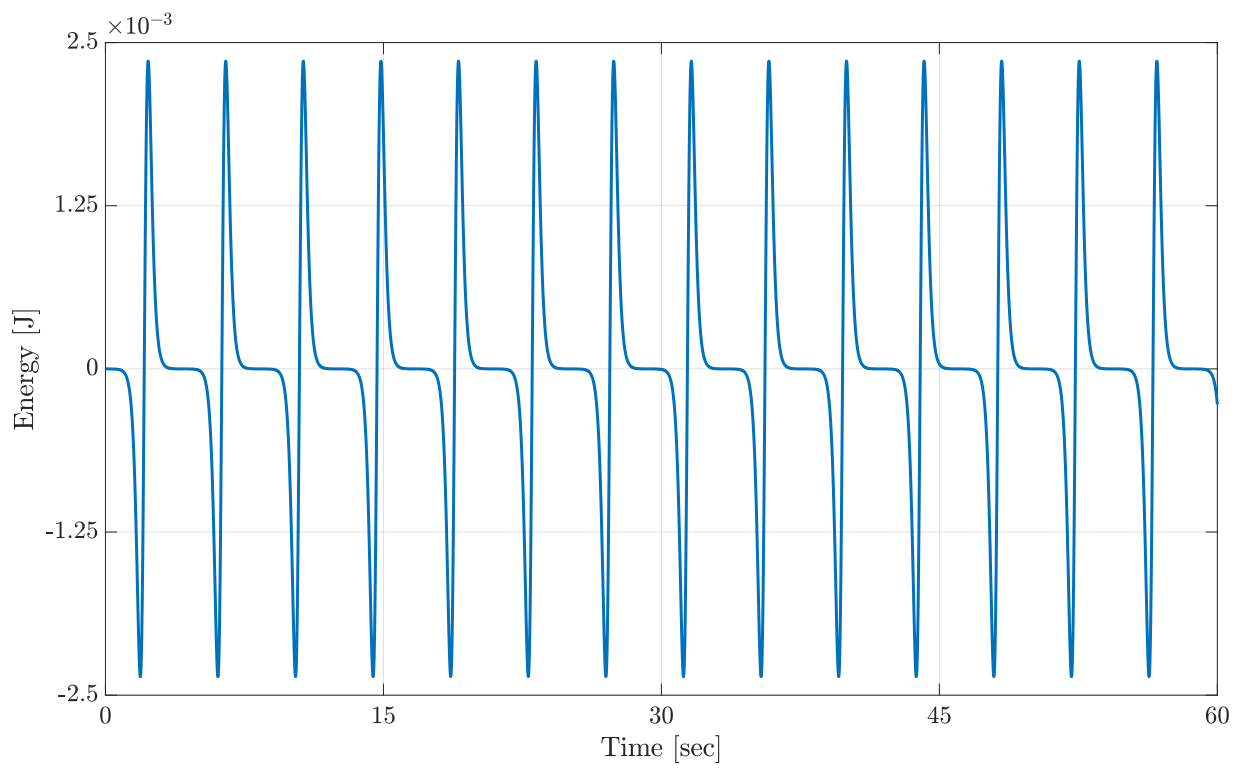

Figure 4-3: Time vs. energy plot of a 1-node whip model. The 1-node whip model was initially positioned 3.13-radian from the vertically-down stable equilibrium posture (i.e. 0.01-radian off from the vertically-upward unstable equilibrium posture). Detailed whip parameters: $(N, l, m, k, b)=(1,1,1,0,0)$

Since there was no dissipative element in the model, the energy of the lossless 1-node whip model should remain constant. Using the (semi-implicit) Euler method, although the energy value itself fluctuated along time, the value was still bounded within a finite range, and the mean energy value vs. time neither increased nor decreased [Figure 4-3]. Even though long-term instability of the simulation might occur due to accumulation of numerical errors of the integrator, for a single degreeof-freedom model, the (semi-implicit) Euler method resulted in a stable simulation without any sign of unbounded divergence of the energy value.

As we increased the number of nodes $N$ and tested with different initial positions, 
the law of energy conservation was violated. Compared to the previous result where the energy value was finite-bounded with respect to time, a gradual increase of energy value was observed for whip models with more than 1 degree-of-freedom $(N>1)$ [Figure 4-4]. The accumulated error innate in the numerical integrator acted as equivalent to a negative damper in the simulation. The unbounded growth of energy, due to the negative damper, resulted in instability of the simulation.

Note that the rate of growth of mean energy, and the amplitude of local fluctuations of energy, were (approximately) proportional to the node number $N$. This result implied that the accumulation of numerical error became proportionally larger as the number of degrees-of-freedom of the system increased.



Figure 4-4: Plot of total energy vs. time with different lossless whip models. Values for mass $m$, length $l$, torsional stiffness coefficient $k$ and torsional damping coefficient $b$ were fixed, and different values of node number $N$ were tested. The straightened $N$-node whip model was initially positioned 0.3-radian from the vertically down equilibrium posture, and was released to freely oscillate. Whip parameters: $(l, m, k, b)$ $=(0.1,0.1,0,0)$. 


\subsubsection{Method 2-Displacement Function Excitation}

The energy of a lossless $N$-node whip model should remain constant after the excitation ended. However, using the (semi-implicit) Euler method as the numerical integrator for the MuJoCo simulation, a gradual decrease of total energy was observed. Consistent with the previous result, the simulated energy loss was more pronounced for models with more than 1 degree-of-freedom $(N>1)$. This apparent dissipation of total energy, due to the accumulation of numerical error, acted as an equivalent positive damper in the simulation [Figure 4-5].

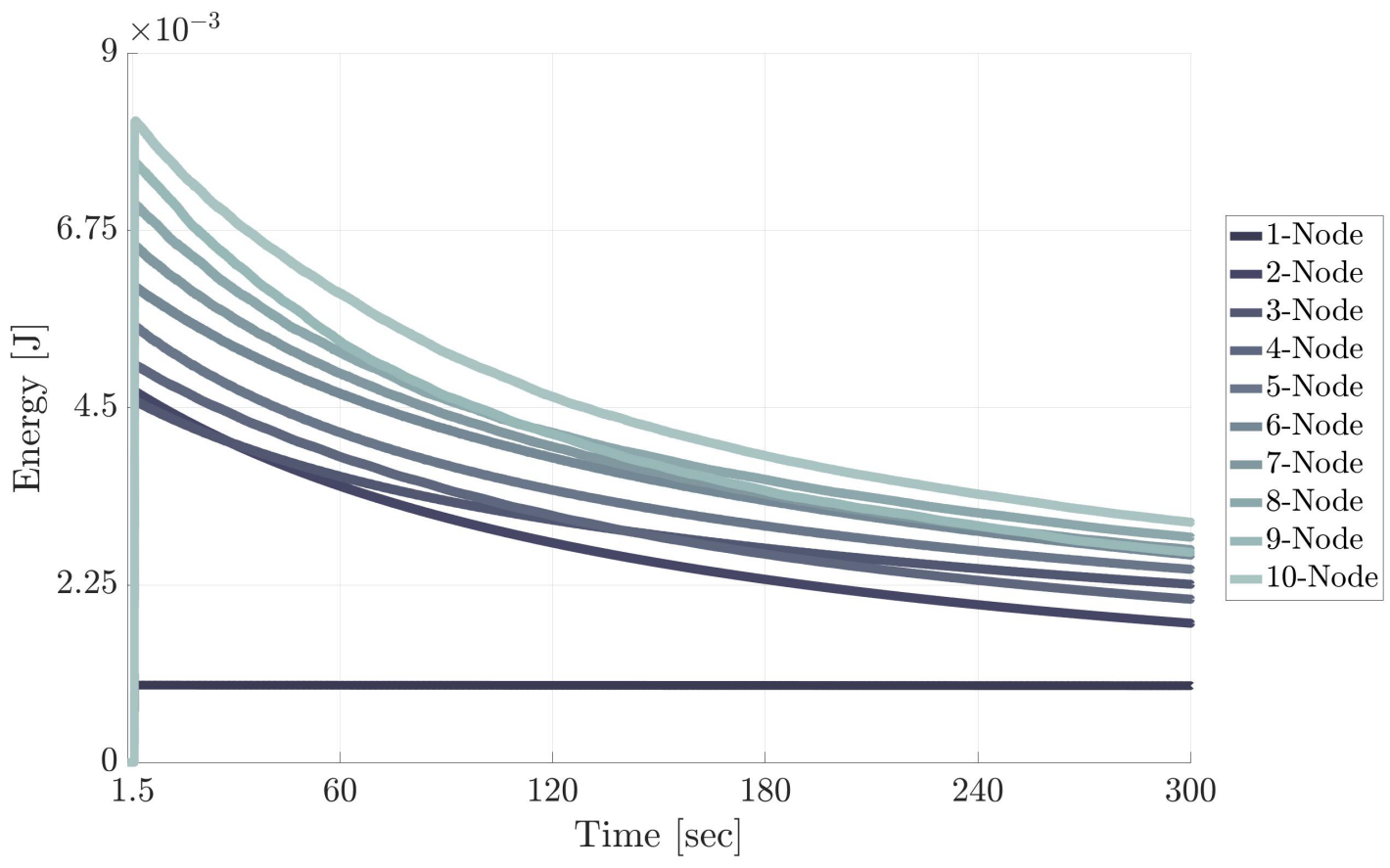

Figure 4-5: Numerical dissipation of total energy over time with different lossless whip models. Excitation with displacement function $p(t)$ started at $1.5 \mathrm{~s}$. Values for mass $m$, length $l$, torsional stiffness coefficient $k$ and torsional damping coefficient $b$ were fixed, and different values of node number $N$ were tested. Whip parameters: $(l, m, k, b)=(0.1,0.02,0.05,0)$; displacement function parameters: $\left(A, t_{0}, w\right)=$ $(0.02,1.5,0.1)$ [Eqn. 4.1]. 


\subsection{Conclusion}

It was discovered that for whip models with more than one degree-of-freedom $(N>$ 1), the law of energy conservation was violated with MuJoCo's semi-implicit Euler method. Positive and negative numerical damping were observed using different methods and whip models. Under what configuration the simulation would exhibit a positive or negative numerical damping remains to be answered.

To improve the physical fidelity of the simulation, it is important to compensate the error due to the innate numerical damping of the simulator. Since numerical dissipation acts as intrinsic damping within the simulation, dissipation in the whip model is the sum of implicit numerical dissipation and explicit model dissipation (due to non-zero $b$ ). Hence, carefully choosing the damping value $b$ may compensate for the numerical error intrinsic to the simulation, such that the combination provides a reliable approximation of the actual whip behavior. 


\section{Chapter 5}

\section{Analysis of a Linearized Whip Model}

Theoretical analysis of the discretized $N$-node planar whip model [Section 3.1] was conducted. Based on Euler-Lagrange equations, it is straightforward (albeit tedious) to establish the governing equations of the discretized $N$-node whip model. However, owing to the complexity of the dynamics, closed-form or analytical solutions for whip models with more than two degrees-of-freedom are impractical. Even the double pendulum exhibits a rich variety of motions, including chaotic behavior [Shinbrot et al., 1992].

However, if the motion is restricted to small deviations from the equilibrium configuration, the nonlinear equations of motion of the $N$-node whip model can be linearized, and linear analytical methods can be used for system analysis [Braun, 2003]. By describing the dynamics of the linearized $N$-node whip model in a state-space representation, information on system responses and their sensitivity to model parameters can be derived. 


\subsection{Method}

Nonlinear Euler-Lagrange equations of motion were linearized about rest in the verticallydownward position, which yielded the following state-space representation:

$$
\dot{x}=A x:=\left[\begin{array}{cc}
0_{n \times n} & I_{n \times n} \\
-M_{w}^{-1}\left(K_{w}+G_{w}\right) & -M_{w}^{-1} B_{w}
\end{array}\right]\left[\begin{array}{c}
q \\
\dot{q}
\end{array}\right]
$$

where $\boldsymbol{M}_{\boldsymbol{w}}, \boldsymbol{B}_{\boldsymbol{w}}, \boldsymbol{K}_{\boldsymbol{w}}, \boldsymbol{G}_{\boldsymbol{w}} \in \mathbb{R}^{n \times n}$ are matrices of inertia, damping, stiffness and gravitational effects, respectively; $\mathbf{0}_{\boldsymbol{n} \times \boldsymbol{n}}, \boldsymbol{I}_{\boldsymbol{n} \times \boldsymbol{n}} \in \mathbb{R}^{n \times n}$ are the zero and identity matrices, respectively; vector $\boldsymbol{q} \in \mathbb{R}^{n}$ denotes joint angles in relative angle coordinates; $\boldsymbol{A} \in \mathbb{R}^{2 n \times 2 n}$ is the state matrix and $\boldsymbol{x} \in \mathbb{R}^{2 n}$ is the state vector. Detailed derivation of the state-space representation and matrices is provided in [Appendix A].

An eigenvalue and eigenvector analysis of state-space matrix $\boldsymbol{A}$, with different values of torsional damping coefficient $b$, was conducted to study the effect of damping on the whip dynamics. The real (dissipative) and imaginary (oscillatory) components of the eigenvalues of matrix $\boldsymbol{A}$ were evaluated for different values of $b$. The imaginary part of the complex eigenvalue was called as the (eigen)frequency of the eigenvalue, and the corresponding eigenvector was called as the (eigen)mode of the corresponding eigenvalue.

To check whether the dynamic behavior of the whip shown in the simulation was consistent with the eigenvalue and eigenvector analysis, spectral analysis of the whip model was conducted. To excite the (eigen)frequencies of the whip model, the displacement function excitation method [Section 4.2] was used on the planar $N$ node whip model. The position controller moved the top of the modeled whip in a horizontal motion from rest with a position input described by [Eqn. 4.1]. For 5 minutes, the relative angle data of the $N^{t h}$ joint (i.e. the angle of the last-suspended sub-model of the whip model) were extracted with a sampling frequency of $500 \mathrm{~Hz}$.

In $\mathrm{MuJoCo}$, the relative angle of the joint was extracted by calling qpos array under mjData. Using the Fast Fourier Transform (FFT) of the Numpy python library, a spectral analysis was conducted on a segment of the data. 


\subsection{Result}

\subsubsection{Pole-Zero Analysis}

As the damping value $b$ changed, while the other parameters were fixed, the complex eigenvalues of matrix $\boldsymbol{A}$ which were located further from the complex plane origin and had proportionally larger real (dissipative) components [Figure 5-1]. As a result, (eigen)modes with higher (eigen)frequency (i.e. complex eigenvalues with larger imaginary parts) decayed faster than (eigen)modes with lower (eigen)frequency (i.e. complex eigenvalues with smaller imaginary parts). In other words, the highfrequency oscillatory behavior of the damped whip model quickly faded, and the whip behavior was dominated by (eigen)modes with the lowest frequencies [Mottershead, 2016].

\subsubsection{Spectral Analysis}

The theoretical result of the pole-zero analysis was confirmed by spectral analysis. When a non-zero damping coefficient value $b$ was included in the whip model, the dominant dynamic behavior was a single frequency of oscillation, which was shown as a single dominant peak in the power spectrum [Figure 5-2]. The dominant frequency value of the damped whip model was $1.175 \mathrm{~Hz}$, which was in reasonable agreement with the theoretical value, $1.168 \mathrm{~Hz}$. Whether the small difference of value $(0.007 \mathrm{~Hz})$ was due to the numerical dissipation within the MuJoCo, or due to the error from the linearization, remains to be determined. 


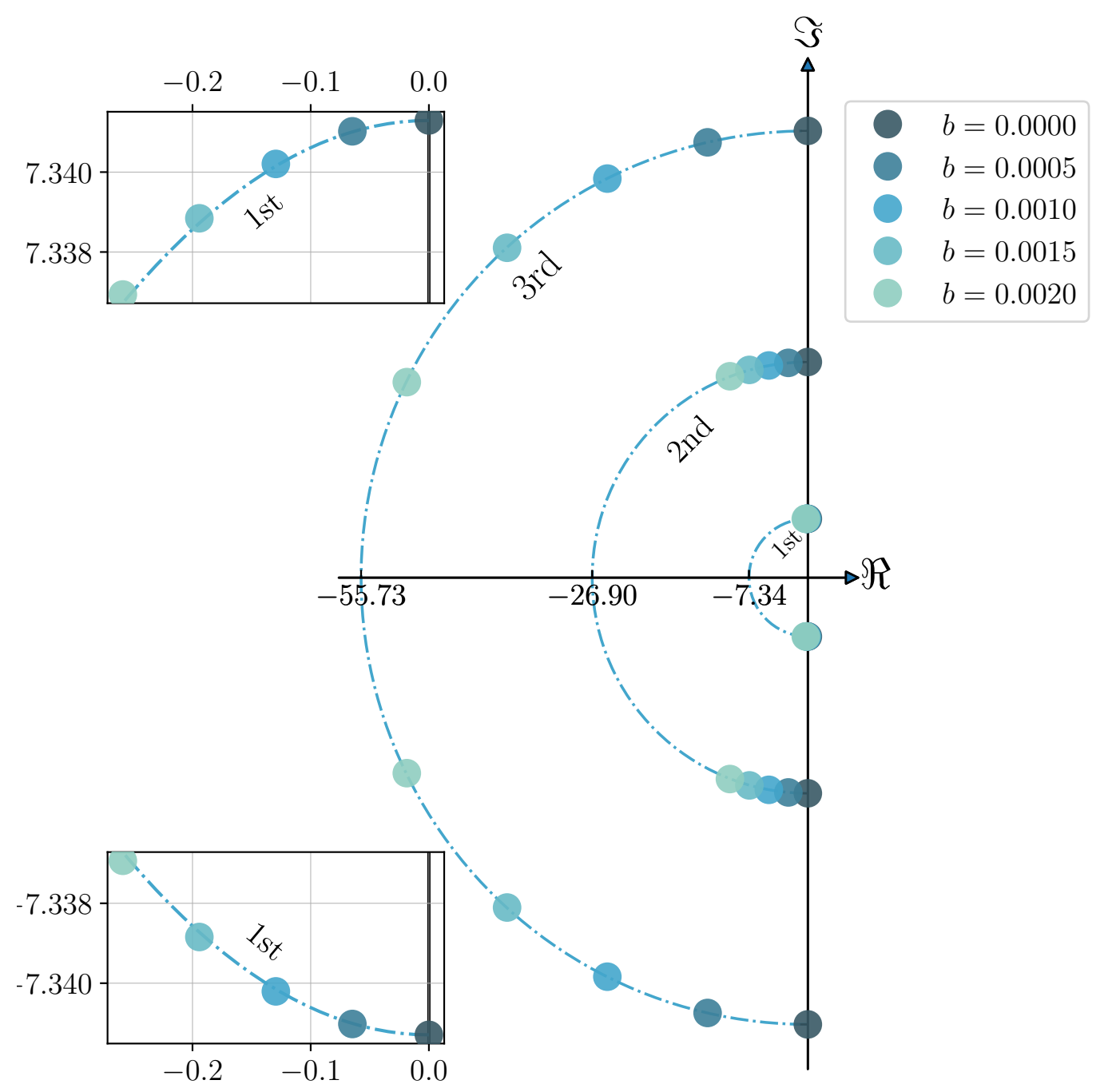

Figure 5-1: Plot of the (complex) eigenvalues of matrix $\boldsymbol{A}$ in the complex plane. The radial grids are numbered in ascending order of distance from the origin. Whip parameters: $(N, l, m, k)=(3,0.1,0.02,0.05)$. 


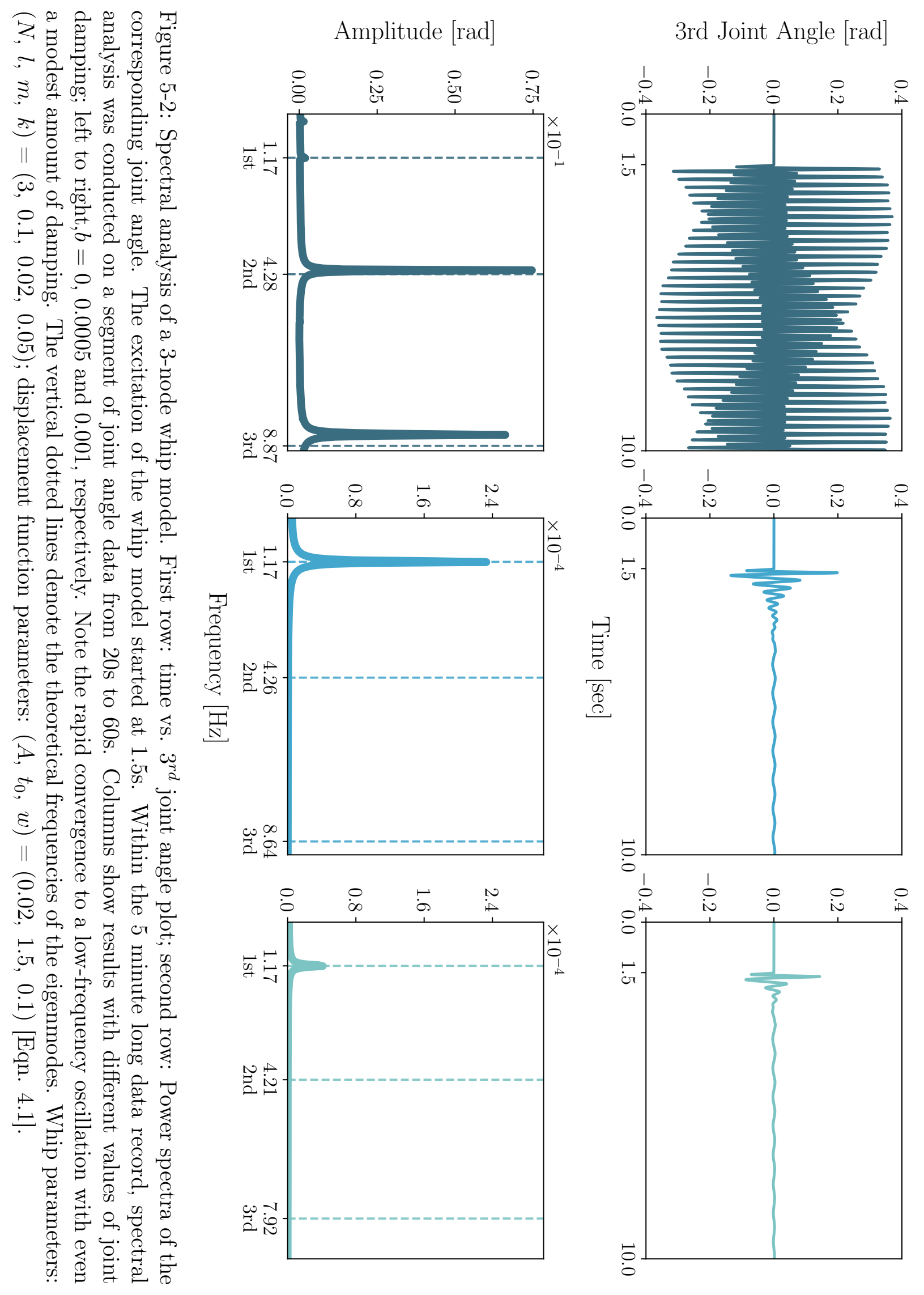




\subsection{Conclusion}

Theoretical analysis of a linearized $N$-node planar whip model showed that the torsional damping coefficient $b$ highly affected the resulting dynamics. Theoretically, the dynamics of the linearized $N$-node whip model is a superposition of $N$ distinct (eigen)modes. However, even a modest amount of damping simplified the complex whip dynamics - the dynamics was dominated by few of the lowest (eigen)frequencies and their corresponding (eigen)modes. This theoretical result was important to determine the estimated whip parameters from the experiment [Section 6.1], and to understand the robustness analysis of the optimal solution [Section 7.5]. Within a range of motion where errors due to linearization (the small-angle approximation) of the model are negligible, the difference between the theoretical frequency value and the frequency derived from spectral analysis was most likely due to numerical damping within the simulation. The model damping parameter may be selected to compensate for this numerical artifact. 


\section{Chapter 6}

\section{Experimental Estimation of Whip}

\section{Parameters}

A simple experiment was designed to estimate the 5 parameters of the planar whip model, $(N, l, m, k, b)$ [Section 3.1]. The experiment was conducted at Action Lab in Northeastern University, by Aleksei Krotov and Marta Russo. In this experiment, 3D position tracking markers attached on the whip were used for the parameter estimation. By releasing the whip from rest at some initial position, the resulting frequency and exponential decay time-constant of the oscillation were measured, and these values were used to estimate the whip parameters. From the experimentally estimated whip parameters, the "experimentally-fitted" whip model was constructed in simulation, and the accuracy of the estimated parameters of the whip model was examined.

\subsection{Method}

A commercially available $1.8 \mathrm{~m}$ bullwhip was used for the experiment. The distal parts of the whip, fall and cracker $^{1}$, were removed. 12 markers were attached to the whip for 3D position tracking: 2 markers at the handle and 10 customized reflective

\footnotetext{
${ }^{1}$ The fall is a single piece of tapered leather suspended from the main part of the whip. The cracker is a twisted piece of short rope suspended from the fall of the whip. The cracker is believed to be important to produce the supersonic crack of the whip.
} 
markers mounted at equal distances along its 1.8m length main part [Figure 6-1].

(a)



(b)

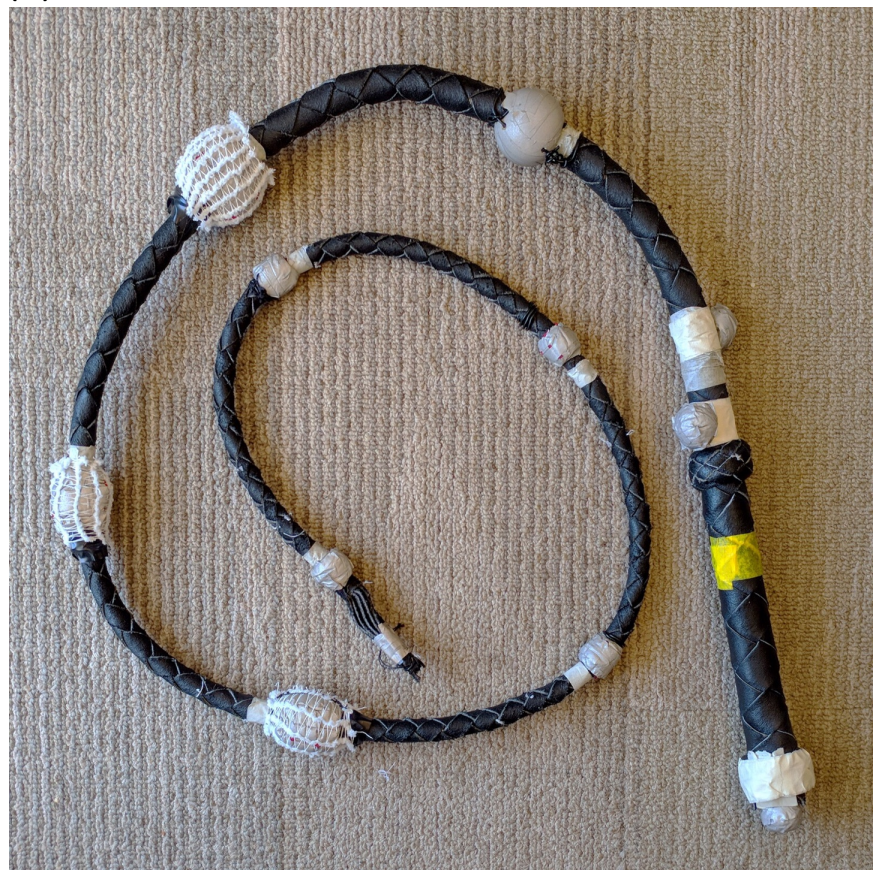

Figure 6-1: (a) Illustration of the actual whip. The green-shaded region depicts the handle of the whip; the yellow-shaded region depicts the main part of the whip; the circles attached to the whip illustrate the markers used for position tracking. (b) Customized $1.8 \mathrm{~m}$ bullwhip used for the experiment. In total, 12 markers were mounted on the whip.

For parameter estimation, it was assumed that (1) the physical properties of the whip were isotropic (i.e. had the same value of stiffness $k$ and damping $b$ when measured in different directions), and (2) the mass was uniformly distributed along the whip length without tapering. Considering the physical properties of the actual whip, these assumptions are demonstrably incorrect. The mass per unit length of the actual whip tapered from handle to tip; intertwined straps of leather which composed the main part of the whip produce anisotropic physical properties. However, these two assumptions were made to avoid cluttering the simulation model with excessive detail.

With the given number of nodes $N$ for the experimentally-fitted whip model, the mass $m$ and length $l$ of the sub-models were derived from direct measurement of total mass $(0.3 \mathrm{~kg})$ and length $(1.8 \mathrm{~m})$ of the actual whip. 
The values of stiffness $k$ and damping $b$ were indirectly measured from a simple oscillation experiment. The top of the handle was attached to a pivot, and the whip was freely suspended. The tip of the whip was manually displaced approximately $20 \mathrm{~cm}$ horizontally from equilibrium and released to initiate small oscillations. Twelve Oqus cameras (Qualisys, Goetheborg, Sweden) recorded the 3D position of whip markers for 20 seconds at a sampling rate of $500 \mathrm{~Hz}$. The frequency and the exponential decay time-constant of the whip oscillation were identified.

The 2D horizontal position data ( $x$ and $y$ coordinate of the Qualisys Cartesian coordinate frame) of each marker were used to measure the frequency $f[H z]$ of the oscillation. All analyses were conducted using MATLAB (Mathworks Inc., Natick, MA). FFT analysis was conducted on the $x$ and $y$ coordinate position data. The mean value of $x$ and $y$ position data were subtracted for each marker. The dominant frequency values in the frequency domain were averaged across markers to measure the frequency $f$.

With the measured frequency $f$, the exponential decay time-constant $\tau_{\text {decay }}[s]$ of the oscillation was calculated. The position data of the marker at the tip of the whip was used for the calculation. Since the trace of oscillation was not aligned with the $x$ and $y$ axis of the Qualisys Cartesian coordinate frame, the position data were rotated so that the starting position of the marker resided on the $x$ axis.

The rotated $x$ position vs. time was curve-fit with the following function $c(t)$ to find the coefficient of exponential decay $\tau_{\text {decay }}$ :

$$
c(t)=C e^{-t / \tau_{\text {decay }}} \sin (2 \pi f t+\phi)
$$

where $C[\mathrm{~mm}]$ is the amplitude and $\phi[\mathrm{rad}]$ is the phase offset of the sine function. The values of $C, \tau_{\text {decay }}$ and $\phi$ which minimized the sum of squared errors with the experimental data were searched with the fminsearch function.

From the measured frequency $f$ and the exponential decay time-constant $\tau_{\text {decay }}$, the best values of stiffness $k$ and damping $b$ of the whip model were computed which resulted in the same damped oscillation. For searching the optimal $k$ and $b$ values, 
the theoretical analysis of the (eigen)frequency and (eigen)mode of the linearized whip model was considered [Section 5.1]. Approximating the actual whip dynamics as a linearized state-space model, theoretically, the dominant frequency $f$ and its corresponding mode of the damped oscillation would be the lowest (eigen)frequency and (eigen)mode of state matrix $A$ [Eqn. 5.1].

With the given $k$ and $b$ values, the complex eigenvalue of matrix $A$ with smallest imaginary part (i.e. lowest eigenfrequency) was extracted. Since the real (dissipative) and imaginary (oscillatory) part of this complex eigenvalue corresponded to the measured exponential decay time-constant $\tau_{\text {decay }}$ and frequency $f$, the best $k$ and $b$ which matched these values were searched. The 1sqnonlin function was used for iteratively searching the $k$ and $b$ values. Summarizing, the whip model parameters which reproduced the same oscillation frequency $f$ and exponential decay time-constant $\tau_{\text {decay }}$ were determined.

Based on the experimentally estimated whip parameters, the experimentally-fitted whip model was built in the MuJoCo simulator, and a comparison with the actual experimental data was conducted. To emulate the experiment conducted with the actual whip, the initial condition response method [Section 4.1] was conducted with the experimentally-fitted whip model. The simulation was conducted with the same time length and sampling rate as the experiment: for 20 seconds the 3D Cartesian position of the tip of the experimentally-fitted whip model was extracted at the sampling rate of $500 \mathrm{~Hz}$. The 3D Cartesian position of the tip was collected by printing out geom_xpos array under mjData. Function $c(t)$ was curve-fit to the extracted position data, and $f$ and $\tau_{\text {decay }}$ values were compared with the actual whip data. Moreover, the root-mean-square error between the position data of the experimentally-fitted whip model and the actual whip was calculated. 


\subsection{Result}

The experimentally observed trajectory of the 2D horizontal position of a single marker attached at the tip of the whip is shown in [Figure 6-2]. Note that the approximately elliptical 2D trajectory of the oscillation gradually tilted counterclockwise as time progressed. Small oscillations in the $y$ direction were observed in the experimental data.



Figure 6-2: Plot of the 2D horizontal position of the marker attached at the tip of the whip. The position data of $x$ and $y$ coordinates were adjusted to position the mean value of $x$ and $y$ position at the origin. The blue dot denotes the starting position of the oscillation. The $2 \mathrm{D}$ horizontal data were rotated to align the blue marker with the global $x$ axis.

FFT analysis of the 10 markers of the main part of the whip is shown in [Figure 6-3]. Regardless of the coordinate and markers, the dominant frequency was $0.45 \mathrm{~Hz}$. Based on the measured frequency $f$, the exponential decay time-constant $\tau_{\text {decay }}$ was calculated [Figure 6-4]. The exponential decay time-constant, $\tau_{\text {decay }}$ was 29.24s. From the measured frequency $f$ and the exponential decay time-constant 
$\tau_{\text {decay }}$, the 5 whip model parameters were determined. Choosing the number of nodes $N$ as 25 , the lsqnonlin function succeeded to converge to a valid $k$ and $b$ values. ${ }^{2}$ Detailed parameters of the experimentally-fitted whip model are presented in [Table $6.1]$.

\begin{tabular}{|c|c|c|c|c|c|}
\hline & $N$ & $l$ & $m$ & $k$ & $b$ \\
\hline \hline Experimentally-fitted & 25 & 0.072 & 0.012 & 0.242 & 0.092 \\
\hline
\end{tabular}

Table 6.1: Detailed values of the experimentally-fitted whip model. $N[-]$ : number of nodes (i,e, sub-models) for the whip model; $m[\mathrm{~kg}]$ : the point-mass of each sub-model; $l[\mathrm{~m}]$ : length of the sub-model; $k[N \cdot \mathrm{m} / \mathrm{rad}]$ : coefficient of linear torsional spring of the rotational joint; $b[N \cdot m \cdot s / r a d]$ : coefficient of linear torsional damping of the rotational joint.
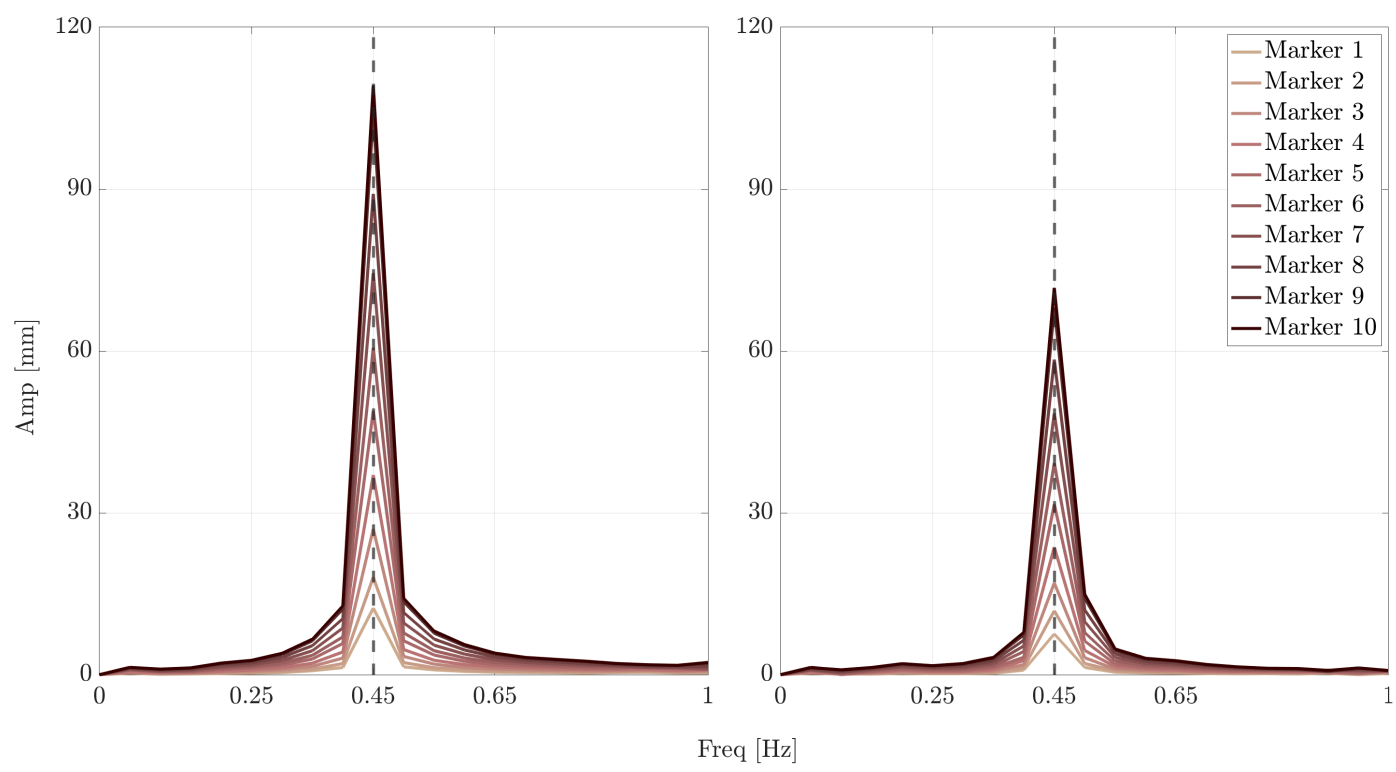

Figure 6-3: (Left) FFT spectral analysis of the $x$ position of the whip markers. (Right) FFT spectral analysis of the $y$ position of the whip markers. Excluding the markers attached to the handle, 10 markers were numbered in ascending order of distance from the handle. The dominant frequency value was $0.45 \mathrm{~Hz}$ for both cases.

\footnotetext{
${ }^{2}$ The value $N=25$ was simply the "sweet-spot" which was manually discovered - the node number $N$ was high enough, and function lsqnonlin converged to a positive $k$ and $b$ values.
} 




Figure 6-4: Plot of the rotated $x$ position data vs. time of the experimental and curve-fit data. The red-dotted line depicts the curve-fit function $c(t)$ [Eqn. 6.1]; the blue-dotted line depicts the corresponding exponential decay curve. Detailed parameters of function $c(t):\left(C, \tau_{\text {decay }}, \phi\right)=(180.07,29.24,-1.062)$.

With the experimentally identified whip parameters, a comparison between the experimentally-fitted whip model and the actual whip was conducted. As shown in [Figure 6-5, 6-6], the exhibited dynamics of the experimentally-fitted whip model were in good agreement with the actual experimental data. The dominant frequency value was exactly the same with the two: $0.45 \mathrm{~Hz}$ for both. The exponential decay time-constant were near the same: $29.24 \mathrm{~s}$ and $29.07 \mathrm{~s}$ for the actual whip and experimentally-fitted whip model, respectively [Figure 6-5]. The root-mean-square error between the position data of experimentally-fitted whip model and the actual whip was 43.65mm [Figure 6-6].

In conclusion, the whip model with experimentally estimated parameters was able to reproduce the essential behavior of the actual whip, which was the damped oscillation with frequency $f$ and exponential decay time-constant $\tau_{\text {decay }}$. 


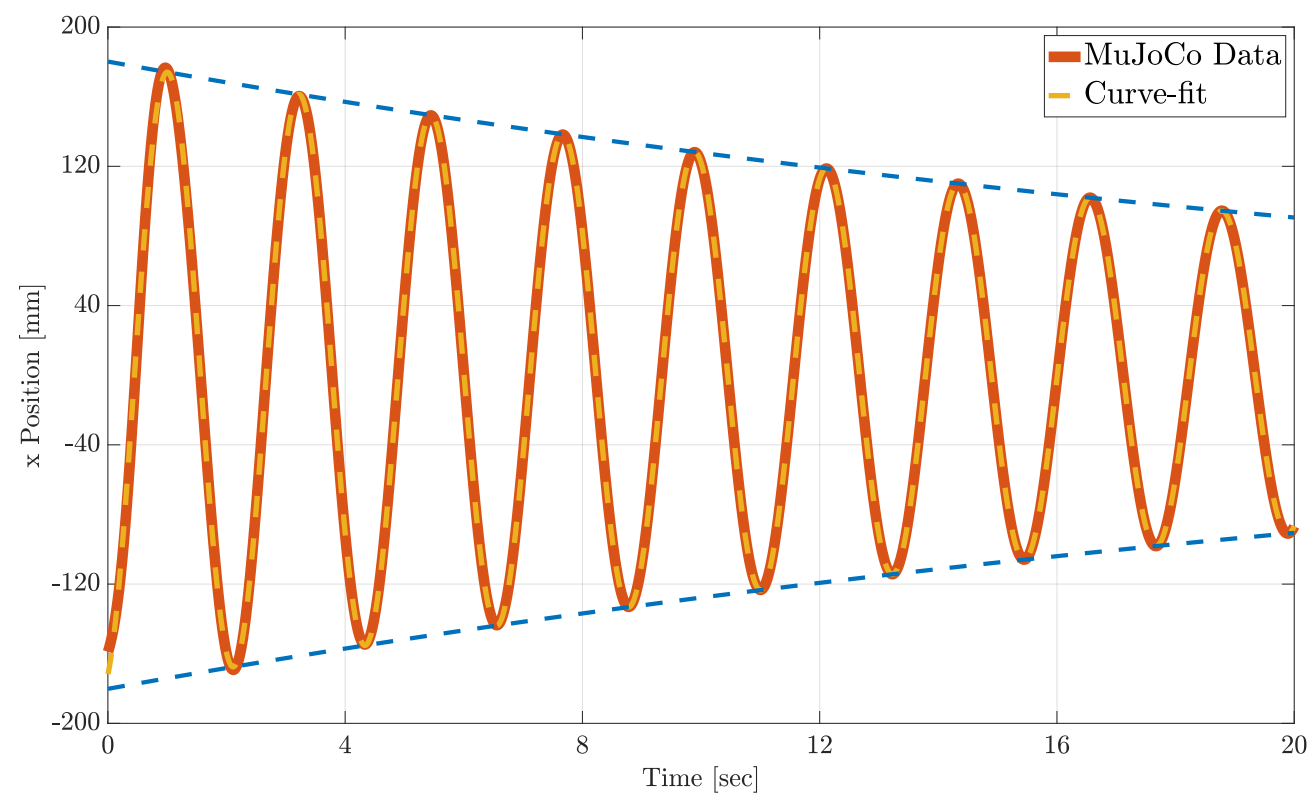

Figure 6-5: Plot of the $x$ position of the tip of the modeled whip vs. time of the MuJoCo and curve-fit data. The yellow-dotted line depicts the curve-fit function $c(t)$ [Eqn. 6.1]; the blue-dotted line depicts the corresponding exponential decay curve. Detailed parameters of function $c(t):\left(C, \tau_{\text {decay }}, f, \phi\right)=(180.19,29.07,0.45,-1.27)$.

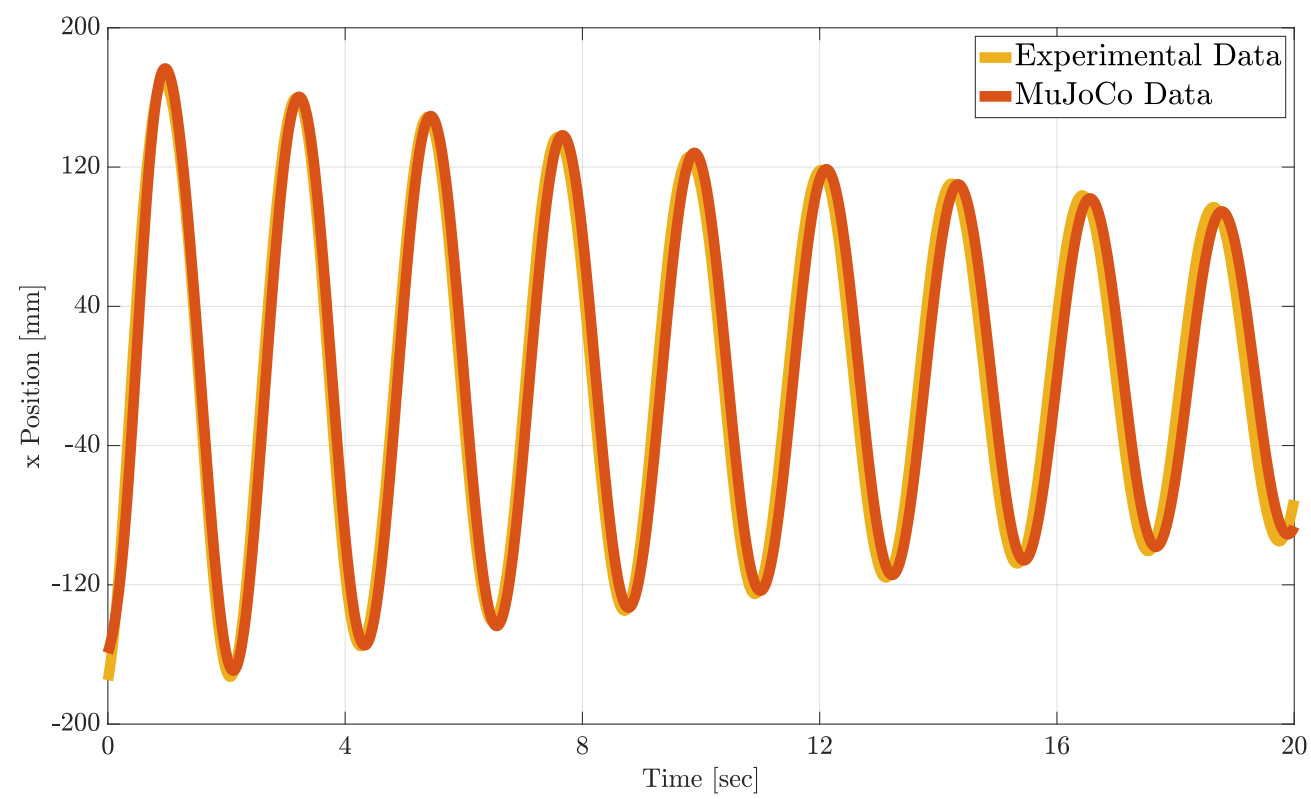

Figure 6-6: Comparison between the actual whip and the experimentally-fitted whip model of MuJoCo. The $x$ position of the tip of the whip for the MuJoCo model and the actual whip are plotted and compared. The root-mean-square error was $43.65 \mathrm{~mm}$. 


\section{Chapter 7}

\section{D Planar Whip Task}

A simple-yet-non-trivial whip task was defined to test the dynamic motor primitives hypothesis. The goal was to reach a distant target with the whip using a single discrete (point-to-point) upper-limb movement. The study tested in simulation whether the task of approximating the target with the whip, could be achieved based on parameterized dynamic motor primitives.

For all of the MuJoCo simulations, the semi-implicit Euler method was chosen as the numerical integrator, with a time step of $0.1 \mathrm{~ms}$.

\subsection{Modeling}

The $N$-node planar whip model [Section 3.1] was connected to the two-segment planar upper-limb model [Section 3.3]. To introduce no torque between the upper-limb and the $N$-node whip model, the whip and the upper-limb were connected with a freelyrotating hinge. This connection was established in simulation by setting the torsional stiffness $k$ and damping coefficients $b$ of the sub-model, which directly attached to the upper-limb model, as zero. Summarizing, the combined model simulated a sequential open-chain planar mechanism with $N+2$ degrees-of-freedom [Figure 7-1]. 

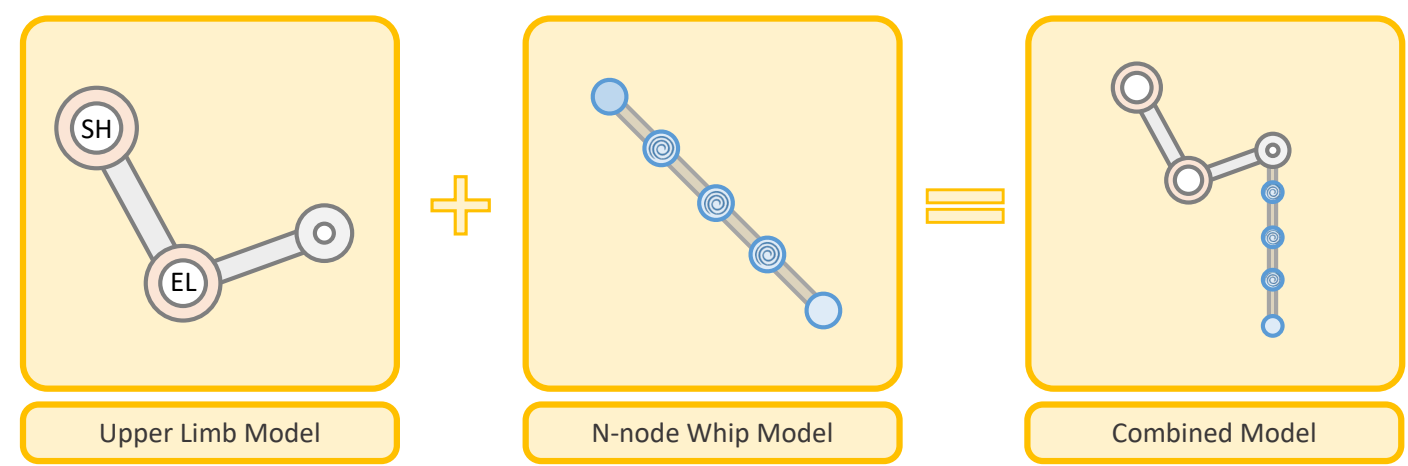

Figure 7-1: The model used for the planar whip task. The planar upper-limb and planar whip model were combined. SH and EL denote shoulder and elbow joint, respectively.

\subsection{Control of the upper-limb}

\subsubsection{Impedance Controller}

To account for physical interaction between the upper-limb and the whip, the model included a simple impedance controller [Hogan, 1985]. The two-joint manipulator muscle model from Flash [Flash, 1987] was adopted and reconfigured:

$$
\boldsymbol{\tau}=\boldsymbol{K}(\boldsymbol{\phi}-\boldsymbol{\theta})+\boldsymbol{B}(\dot{\boldsymbol{\phi}}-\dot{\boldsymbol{\theta}})+\boldsymbol{\tau}_{G}
$$

In this equation, $\boldsymbol{K} \in \mathbb{R}^{2 \times 2}$ is a constant joint stiffness matrix, $\boldsymbol{B} \in \mathbb{R}^{2 \times 2}$ is a constant joint damping matrix, which both represent the neuromuscular mechanical impedance of the upper-limb; vector $\boldsymbol{\tau}(t)=\left[\tau_{1}(t), \tau_{2}(t)\right]^{T}$ denotes the net torque input on each joint; subscripts 1 and 2 denote shoulder and elbow, respectively; vector $\boldsymbol{\tau}_{G}(t)=\left[\tau_{1, G}(t), \tau_{2, G}(t)\right]^{T}$ denotes the torque required for gravity compensation; vector $\boldsymbol{\theta}(t)=\left[\theta_{1}(t), \theta_{2}(t)\right]^{T}$ denotes the actual joint angle trajectory defined in relative angle coordinates [Figure 7-2]; vector $\phi(t)=\left[\phi_{1}(t), \phi_{2}(t)\right]^{T}$ represents a motion command from the CNS as a zero-torque trajectory, i.e. neglecting gravitation effects, if the actual joint angle trajectory $\boldsymbol{\theta}$ exactly matches with the zero-torque trajectory $\phi$, no torque will be exerted by the actuators. 


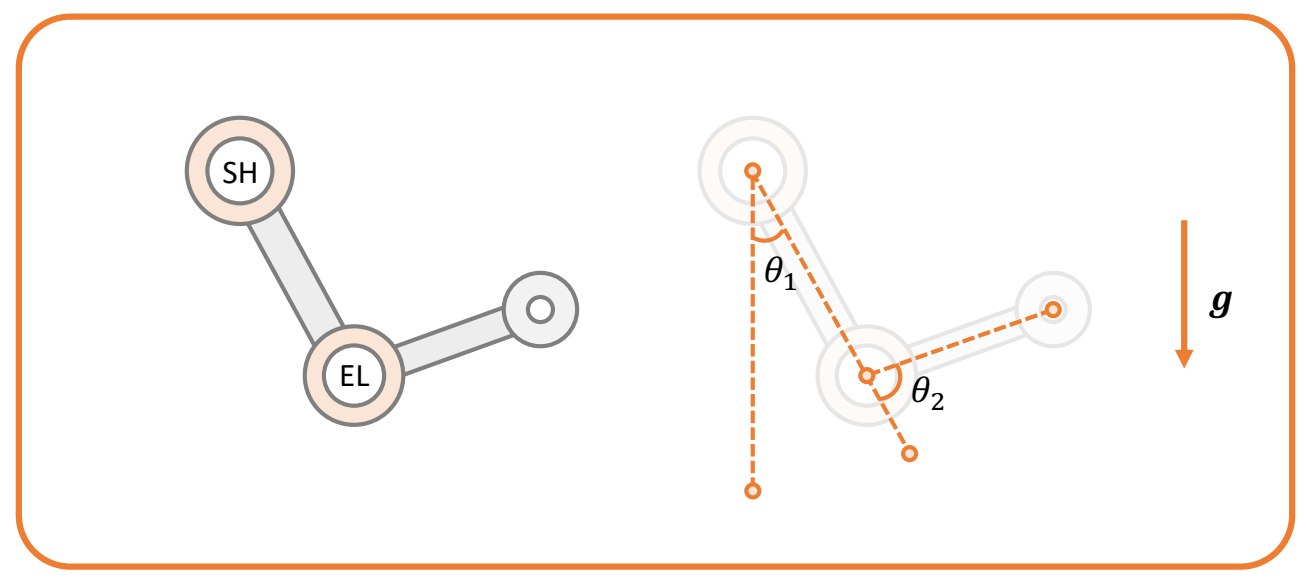

Figure 7-2: The relative angle coordinate used for the planar upper-limb model. Angle $\theta_{1}(\mathrm{rad})$ and $\theta_{2}(\mathrm{rad})$ denote relative angles of the shoulder and elbow joint, respectively.

Detailed values for $\boldsymbol{K}$ and $\boldsymbol{B}$ were borrowed from Flash [Flash, 1987]:

$$
\boldsymbol{K}=\left[\begin{array}{ll}
29.50 & 14.30 \\
14.30 & 39.30
\end{array}\right], \quad \boldsymbol{B}=\left[\begin{array}{ll}
2.950 & 1.430 \\
1.430 & 3.930
\end{array}\right]
$$

Biological observations from previous studies were considered to determine the controller of the planar upper-limb model. Although muscle force production is a complex function of many factors, its interactive behavior can well be described as a function of muscle length and its rate of change [Joyce et al., 1969, Rack and Westbury, 1969, Flash, 1987]. The joint torques resulting from activation of relevant muscles were assumed to depend upon the position and velocity deviation between the actual upper-limb position and a zero-torque position. As the upper-limb controller was described in relative angle coordinate, the stiffness matrix $\boldsymbol{K}$ was taken to be constant, since it was shown that joint stiffness was (approximately) constant in joint coordinates [Flash and Mussa-Ivaldi, 1990]. Based on reports showing that the stiffness field of the upper arm was nearly curl-free and predominantly spring-like, the stiffness matrix $\boldsymbol{K}$ was chosen to be symmetric [Mussa-Ivaldi et al., 1985]. Consistent with a single time-constant characterizing neuromuscular interactive dynamics, values for the joint damping matrix $\boldsymbol{B}$ were assumed to be proportional to the joint 
stiffness matrix $\boldsymbol{K}$ [Flash, 1987]. This set of observations served as the basis for the constructed upper-limb controller, which resulted in a motion resembling the actual motor behavior of the upper-limb.

Gravitational effects were compensated with $\boldsymbol{\tau}_{G}$, so that the actual upper-limb posture $\boldsymbol{\theta}$ could exactly match with the zero-torque posture $\boldsymbol{\phi}$ when the whole model was at rest. In detail, the following equation was used for the gravity compensation torque $\tau_{G}$ :

$$
\boldsymbol{\tau}_{G}=\boldsymbol{J}_{\mathbf{0 1}}^{T} \boldsymbol{f}_{1, G}+\boldsymbol{J}_{\mathbf{0 2}}^{T} \boldsymbol{f}_{2, G}+\boldsymbol{J}_{\mathbf{0 3}}^{T} \boldsymbol{f}_{3, G}
$$

where $\boldsymbol{J}_{\boldsymbol{i} \boldsymbol{j}} \in \mathbb{R}^{3 \times 2}$ is a Jacobian matrix of frame $j$ relative to frame $i ; \boldsymbol{f}_{i, G} \in \mathbb{R}^{3}$ denotes the gravitational force applied to frame $i$; frame 0, 1, 2 and 3 are attached to the shoulder, center of mass of the upper arm, center of mass of the forearm, and the end-effector of the upper-limb model where the connection with the whip happened, respectively [Figure 7-3].



Figure 7-3: Frames and the imposed forces of the simulation model.

The detailed force vectors for each frame were as follows:

$$
\boldsymbol{f}_{1, G}=M_{1} \boldsymbol{g}, \quad \boldsymbol{f}_{2, G}=M_{2} \boldsymbol{g}, \quad \boldsymbol{f}_{3, G}=m_{w} \boldsymbol{g},
$$


where $M_{1}$ and $M_{2}$ denote the mass of upper arm and forearm, respectively [Table 3.1]; $m_{w}$ denotes the total mass of the whip model, which is the node number of the whip $N$ multiplied by the mass of a single sub-model mass $m, m_{w}=m \cdot N$; vector $\boldsymbol{g} \in \mathbb{R}^{3}$ denotes gravity in the simulation environment.

\subsubsection{Zero-Torque Trajectory}

A single discrete upper-limb movement defined the zero-torque trajectory [Section 2.1]. The zero-torque trajectory of the upper-limb model followed a rest-to-rest minimum-jerk profile [Flash and Hogan, 1985] in joint coordinates [Figure 7-4]:

$$
\begin{aligned}
& \phi_{1}(t)=\phi_{1, i}+\left(\phi_{1, f}-\phi_{1, i}\right) \cdot\left\{10\left(\frac{t}{D}\right)^{3}-15\left(\frac{t}{D}\right)^{4}+6\left(\frac{t}{D}\right)^{5}\right\} \\
& \phi_{2}(t)=\phi_{2, i}+\left(\phi_{2, f}-\phi_{2, i}\right) \cdot\left\{10\left(\frac{t}{D}\right)^{3}-15\left(\frac{t}{D}\right)^{4}+6\left(\frac{t}{D}\right)^{5}\right\}
\end{aligned}
$$

where $t$ is time and subscripts $i$ and $f$ denote the initial and final postures, respectively. For times greater than the duration $D$ (i.e. $t>D$ ), the zero-torque trajectory of the shoulder and elbow joint remained at $\phi_{1, f}$ and $\phi_{2, f}$, respectively. Rigorous and detailed mathematical derivation of the minimum-jerk profile is presented in [Appendix B].

Although simple reaches, for which the minimum-jerk-trajectory was first derived, are coordinated as motions of the hand in an extrinsic coordinate frame (e.g., Cartesian) [Morasso, 1981], for simplicity, $\phi(t)$ was defined in joint space. This avoided the need to compute inverse kinematics, which would become a considerable challenge for the spatial task. 


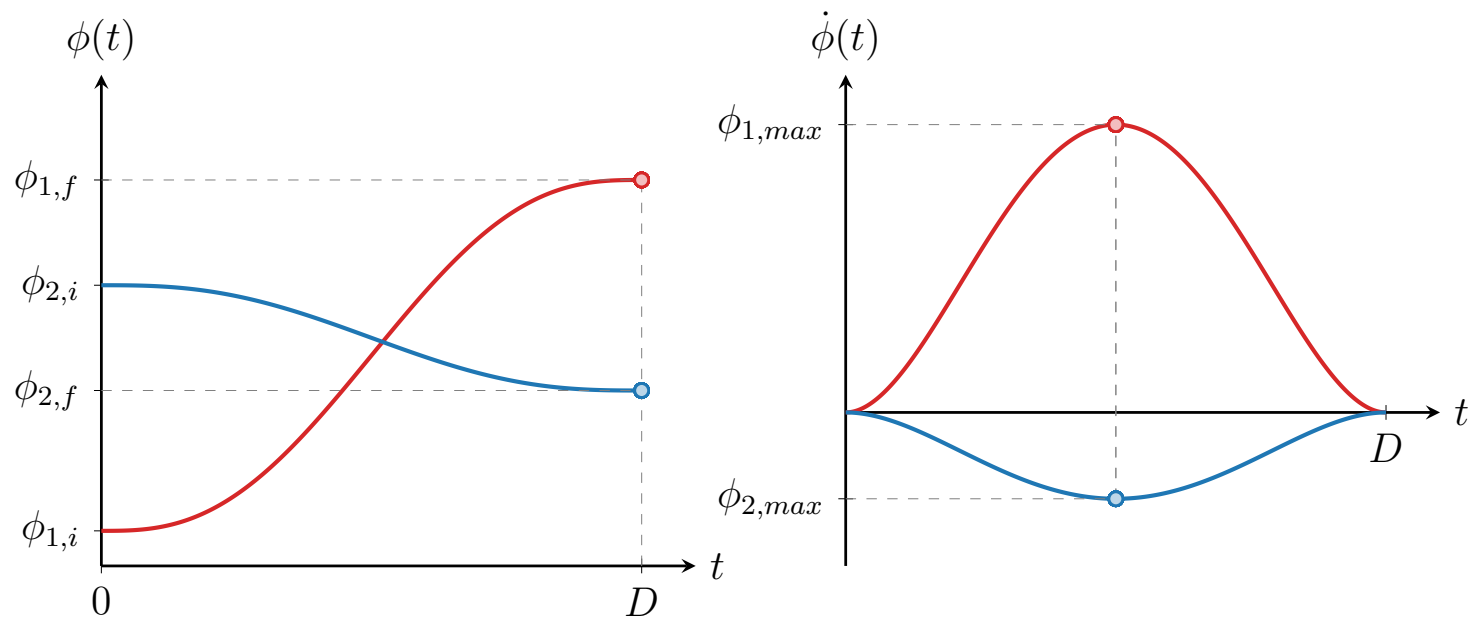

Figure 7-4: Plot of the zero-torque trajectory of the shoulder $\phi_{1}(t)$ and elbow $\phi_{2}(t)$ joint. Detailed values for $\phi_{1, \max }$ and $\phi_{2, \max }$ are $1.875 \cdot\left(\phi_{1, f}-\phi_{1, i}\right)$ and $1.875 \cdot\left(\phi_{2, f}-\phi_{2, i}\right)$, respectively.

A Norton equivalent network representation of the upper-limb controller is shown in [Figure 7-5], and an illustration of the single upper-limb movement is shown in [Figure 7-6].

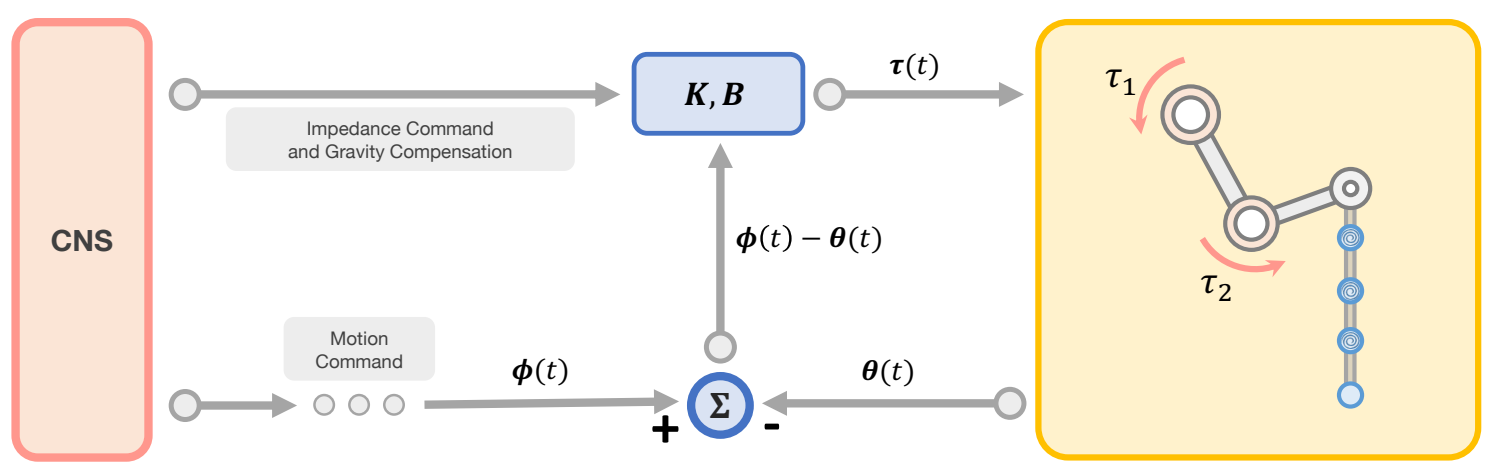

Figure 7-5: The upper-limb controller represented with the Norton equivalent network model. 

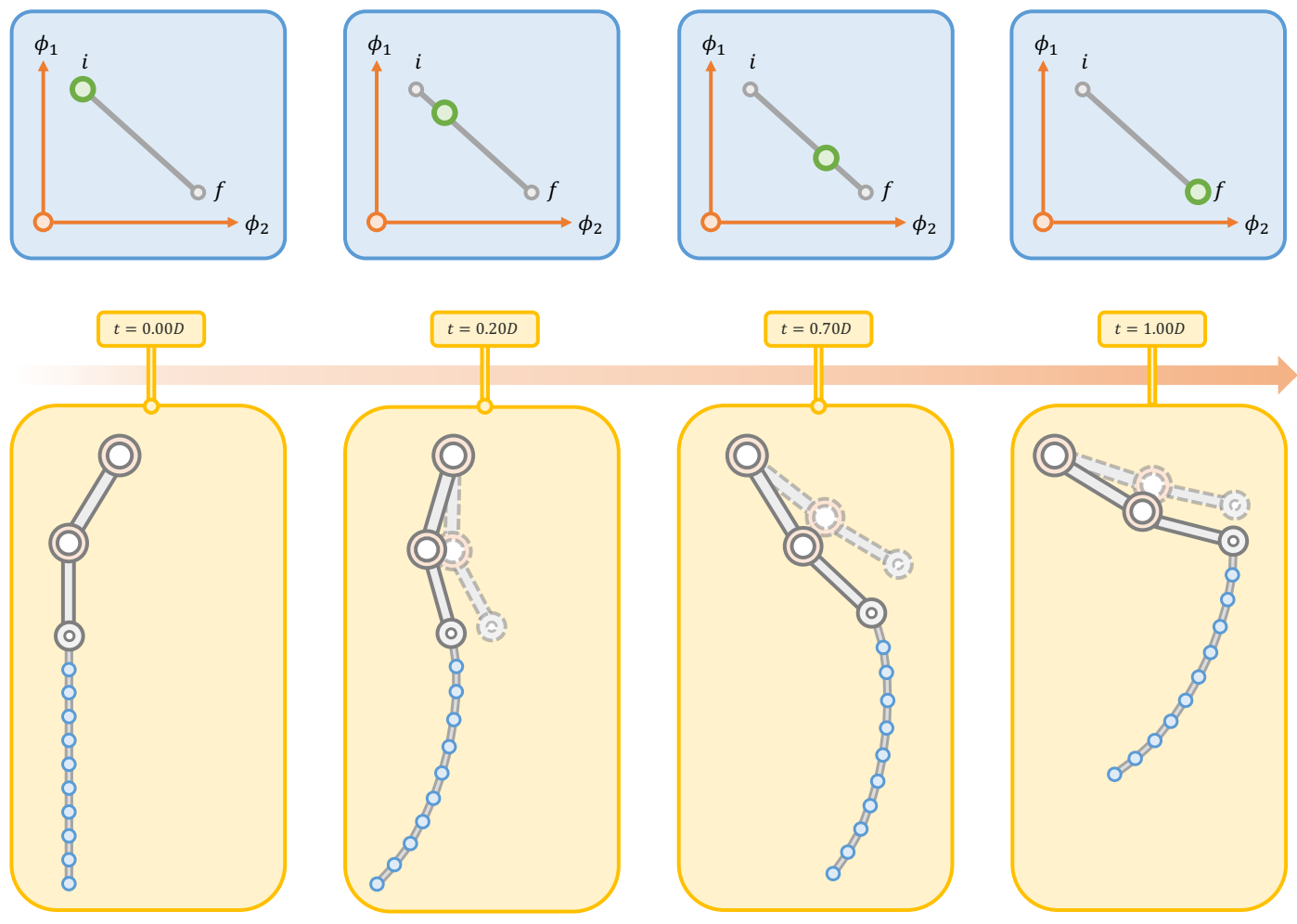

Figure 7-6: Representative images of the zero-torque trajectory and the actual upperlimb movement. The top row depicts the zero-torque trajectory in joint space coordinates. In the bottom row, the transparent upper-limb depicts the zero-torque position of the upper-limb. $t$ denotes time and $D$ denotes the duration of the single discrete upper-limb movement.

\subsubsection{Implementation}

In the simulation, the zero-torque trajectory $\phi(t)$ was determined by 5 movement parameters: $\left(\phi_{1, i}, \phi_{2, i}, \phi_{1, f}, \phi_{2, f}, D\right)$. At every time step, the actual joint angle $\boldsymbol{\theta}$ and angular velocity $\dot{\boldsymbol{\theta}}$ for each joint were extracted by calling qpos and qvel array under mjData. Based on these values, the position and velocity deviation between the zero-torque trajectory $\boldsymbol{\phi}(t)$ and the actual joint angle trajectory $\boldsymbol{\theta}(t)$ were calculated.

The gravity compensation torque $\boldsymbol{\tau}_{G}$ was calculated by calling the Jacobians for each frame with function $m j_{-} j a c B o d y C o m()$ and $m j_{-} j a c G e o m()$. With the specified $\boldsymbol{K}$ and $\boldsymbol{B}$ matrices and the gravity compensation torque $\boldsymbol{\tau}_{G}$, the resultant torque values were calculated and applied to each torque actuator at each time step. 


\subsection{Optimization Method}

The objective of the whip task was to minimize the value $L[m]$, the distance between the tip of the whip and a target, with a single discrete upper-limb movement, i.e. a single set of 5 movement parameters $\left(\phi_{1, i}, \phi_{2, i}, \phi_{1, f}, \phi_{2, f}, D\right)$ [Figure 7-7]. To avoid chaotic behavior due to the model whip colliding with a target, the target was located at a distance just $0.01 \mathrm{~m}$ beyond the combined length of the upper-limb and whip measured from the shoulder $(L 1+L 2+1.8+0.01=2.395 \mathrm{~m})$ [Table 3.1] [Figure 7-8]. The minimum value of the distance $L$ reached with a single discrete (i.e. rest-to-rest) upper-limb movement, $L^{*}[m]$, was a quantitative measure to assess the performance.

For this planar whip task, three variants of the whip model were constructed and tested. Fixing the sub-model of the whip model, short-, medium-, and long-whip models were constructed by increasing the number of nodes (sub-models) $N$. Detailed values of the whip parameters $(N, l, m, k, b)$ for each whip model are listed in [Table 7.1]. The objective of the whip task was cast as an optimization problem: finding the optimal 5 movement parameters $\left(\phi_{1, i}, \phi_{2, i}, \phi_{1, f}, \phi_{2, f}, D\right)$ which resulted the minimum $L^{*}$ value. The distance $L$ was calculated for each time step, by calling the Cartesian positions of the target and the tip of the whip, with function geom_xpos array under mjData.

\begin{tabular}{|c|c|c|c|c|c|}
\hline & $N$ & $l$ & $m$ & $k$ & $b$ \\
\hline \hline Short-whip & 10 & 0.10 & 0.1 & 0.05 & 0.005 \\
\hline Medium-whip & 15 & 0.10 & 0.1 & 0.05 & 0.005 \\
\hline Long-whip & 20 & 0.10 & 0.1 & 0.05 & 0.005 \\
\hline
\end{tabular}

Table 7.1: Detailed values of whip parameters for each whip model. 


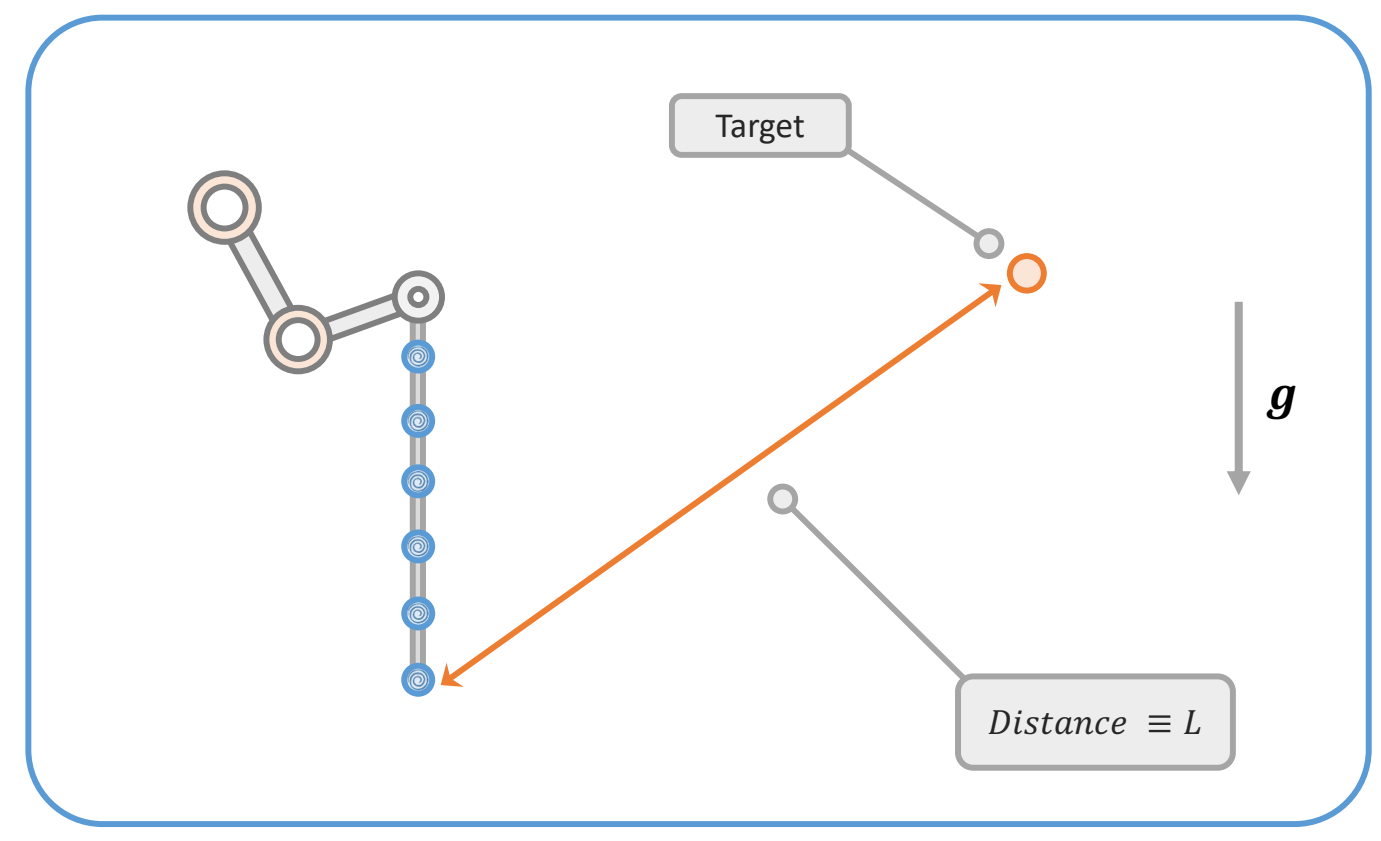

Figure 7-7: The 2D planar whip task.



Figure 7-8: The position of the target. 
For each whip model, the optimal 5 movement parameters $\left(\phi_{1, i}, \phi_{2, i}, \phi_{1, f}, \phi_{2, f}, D\right)$ which minimized $L^{*}$ were identified with the nlopt (nonlinear optimization) $\mathrm{C}++$ tool box. Since the derivative of $L^{*}$ with respect to the five movement parameters $\left(\phi_{1, i}\right.$, $\left.\phi_{2, i}, \phi_{1, f}, \phi_{2, f}, D\right)$ was unknown, several global derivative-free optimization algorithms were compared. Of the global derivative-free optimization algorithm considered, the DIRECT-L (DIviding RECTangles, Locally biased) algorithm was chosen for the optimization [Gablonsky and Kelley, 2001]. A detailed comparison between different global derivative-free optimization algorithms is presented in [Appendix C].

For the DIRECT-L algorithm, the upper and lower bound of the search space were defined. The bounding box constraint for the 5 movements parameters $\left(\phi_{1, i}\right.$, $\left.\phi_{2, i}, \phi_{1, f}, \phi_{2, f}, D\right)$, are presented in [Table 7.2]. Within the bounds of the constraint, the DIRECT-L optimization algorithm conducted 600 iterations.

\begin{tabular}{|c|c|c|c|c|c|}
\hline & $\phi_{1, i}$ & $\phi_{2, i}$ & $\phi_{1, f}$ & $\phi_{2, f}$ & $D$ \\
\hline \hline Lower bound & $-0.5 \pi$ & 0 & 0 & 0 & 0.4 \\
\hline Upper bound & 0 & $\pi$ & $\pi$ & $\pi$ & 1.2 \\
\hline
\end{tabular}

Table 7.2: Lower and upper bound of the 5 movement parameters used for the DIRECT-L optimization algorithm.

\subsection{Optimization and Simulation Results}

For each whip model, the DIRECT-L algorithm converged to an optimal set of five movement parameters which yielded a minimum value of distance $L^{*}$ for the corresponding whip model. Detailed values of the optimal parameter set of the discrete movement, $\left(\phi_{1, i}, \phi_{2, i}, \phi_{1, f}, \phi_{2, f}, D\right)$, and its corresponding output $L^{*}$, are presented in [Table 7.3]. Actual movements in simulation generated with the optimal movement parameters are shown in [Figure 7-9]. A graph of the number of iteration vs. $L^{*}$ with different whip models is shown in [Figure 7-10].

Without the computational impasse of the "curse of dimensionality", all three whip models (based on the same sub-model) converged to an optimal solution. Considering 
the daunting complexity of the whip dynamics, it was not a priori obvious that the optimization would even converge. Nevertheless, encoding the movement based on parameterized dynamic motor primitives simplified the control task and provided a way to work-around the "curse of dimensionality". Regardless of the number of nodes of the whip model, the task of searching for a time-history of inputs to an extremely high degree-of-freedom system (22 degrees-of-freedom system or 44 state variables for the longest whip) was dramatically simplified to searching for 5 movement parameters. This simplification greatly reduced the numerical burden of the control task, and facilitated the optimization process to converge to an optimal solution.

Even though the optimization algorithm used here was able to yield an optimal set of movement parameters, several "spikes" of output value occurred during the optimization process [Figure 7-10]. This phenomenon was due to the DIRECT-L algorithm, which intermittently deviated from a locally-converged value, rather than remaining at it. The spikes occurred when the algorithm "stepped out" from its locally-optimal value (up to that point). These pronounced spikes were due to the structure of the algorithm; the strength of the DIRECT-L algorithm is its balance of global and local search [Gablonsky and Kelley, 2001, Finkel and Kelley, 2004].

\begin{tabular}{|c|c|c|c|c|}
\hline \multirow{2}{*}{\multicolumn{2}{|c|}{ Parameters }} & \multicolumn{3}{c|}{ Whip Type } \\
\cline { 3 - 5 } \multicolumn{2}{|c|}{} & Short-whip & Medium-whip & Long-whip \\
\hline \hline \multicolumn{2}{|c|}{ Total Mass } & 1.0 & 1.5 & 2.0 \\
\hline \multicolumn{2}{|c|}{ Total Length } & 1.0 & 1.5 & 2.0 \\
\cline { 3 - 5 } & $\phi_{i, 1}$ & -1.312 & -1.447 & -1.496 \\
\cline { 2 - 5 } Optimal Movement & $\phi_{i, 2}$ & 1.670 & 0.368 & 0.505 \\
\cline { 2 - 5 } Parameters & $\phi_{f, 1}$ & 1.565 & 1.562 & 1.570 \\
\cline { 2 - 5 } & $\phi_{f, 2}$ & 0.000 & 0.121 & 0.506 \\
\cline { 2 - 5 } & $D$ & 0.667 & 0.833 & 0.803 \\
\hline \hline \multirow{2}{*}{ Optimal Output } & $L^{*}$ & 0.032 & 0.048 & 0.122 \\
\hline
\end{tabular}

Table 7.3: Optimal movement parameters and the corresponding output value $L^{*}$. 600 iterations were conducted with the DIRECT-L algorithm. 

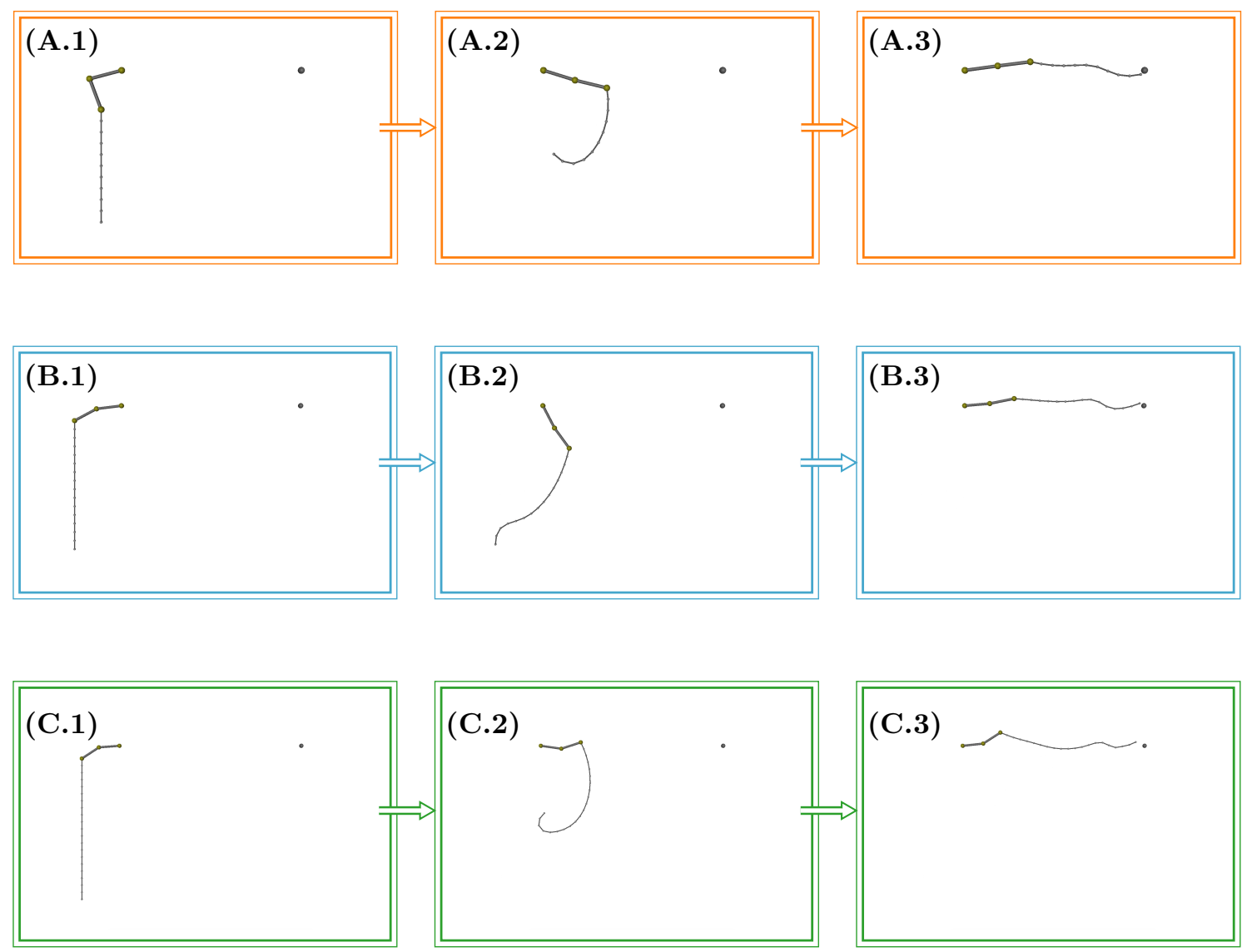

Figure 7-9: Time sequence of the simulation of the whip task. (A) Short-whip (B) Medium-whip (C) Long-whip. Each upper-limb movement was generated with the optimal movement parameters, which yielded the minimum distance $L^{*}$ [Table 7.3]. 







\subsection{Robustness Analysis of the Optimal Solution}

Considering the complexity of the nonlinear whip dynamics, it is likely that the objective function, $L^{*}$ is non-convex and non-smooth with discontinuities. As a result, the objective function $L^{*}$ may have multiple local extrema within the search space. The optimization may become trapped in a local minimum and fail to find the globally optimal solution. This possibility of local minima called for an examination of the "robustness" of the converged optimal solution. Robustness of the converged optimal solution was examined by checking the landscape of the objective function $L^{*}$, in the vicinity of the optimal 5 movement parameters [Table 7.3]. If the landscape of objective function $L^{*}$ in the vicinity of the optimal 5 movement parameters was (relatively) smooth without multiple "valleys" (local minima), the optimal solution was deemed robust.

\subsubsection{Method}

The optimal solution of the short-whip model was chosen for the robustness analysis. Data collected from the 600-iteration optimization process were used for the analysis [Figure 7-10]. To retrieve the 5 movement parameters in the vicinity of the converged optimal value of $L^{*}$, values of $L^{*}$ lower than $0.05 \mathrm{~m}(0.018 \mathrm{~m}$ further from the optimal value of $L^{*}$, which was $0.032 \mathrm{~m}$ [Table 7.3]) and the corresponding 5 movement parameters, were collected. The mean, standard deviation, minimum, maximum, coefficient of variation and standard error values of the collected 5 movement parameters were calculated. ${ }^{1}$ To check the landscape of the objective value $L^{*}$ in the vicinity of the optimal 5 movement parameters, the search space in the vicinity of the optimal solution was taken to be a 5-dimensional hypercube, where each edge ranged between the minimum and maximum value of the collected 5 movement parameters. Within this 5-dimensional hypercube search space, the values of $L^{*}$ were analyzed.

The simplest method to analyze the value of $L^{*}$ was to densely sample the data

\footnotetext{
${ }^{1}$ Coefficient of variation (C.O.V.) is defined as the ratio of the standard deviation $\sigma$ to the mean $\mu$, i.e. C.O.V. $=|\sigma / \mu|$. Standard error (S.E.) is the estimate of the standard deviation of a sample. With the sample size of $N$, S.E. $=\sigma / \sqrt{N}$
} 
points within this 5-dimensional hypercube. However, even for a 5-dimensional search space, due to the "curse of dimensionality", an exhaustive dense sampling of the data points would be demanding. For instance, if 15 points were evenly sampled within the range of each 5 movement parameters, $15^{5}=759,375$ data points in total, would be sampled. Since collecting a single data point corresponded to running a single simulation, assuming 3 seconds were needed for a single simulation, it would require roughly $3 \times 759,375 s=2,278,125 s \approx 26$ days to complete the data acquisition. Hence, a naïve approach of exhaustively collecting the data points was avoided owing to the exponential growth of computation time.

To solve this problem, the strategy of "divide-and-conquer" (Divide et impera), was used for the analysis. Within the 5 movement parameters, 2 movement parameters with the highest standard deviation (i.e. most widely dispersed) were chosen, and the other 3 movement parameters were fixed at the mean value of the collected data. The landscape of output value $L^{*}$ with respect to these two movement parameters was analyzed.

\subsubsection{Result}

The calculated mean, standard deviation, minimum, maximum, coefficient of variation and standard error values of the collected 5 movement parameters are shown in [Table 7.4]. The two parameters with the highest standard deviation were the initial posture of the zero-torque trajectory of the shoulder joint $\phi_{1, i}$ and elbow joint $\phi_{2, i}$. The landscape of output values $L^{*}$ with respect to these two variables is shown in [Figure 7-12]. Even with the highly nonlinear dynamics of the whip, the landscape of output values $L^{*}$ with respect to the initial posture was (relatively) smooth. No local valleys, which could yield local minima, were evident in the plotted landscape. 

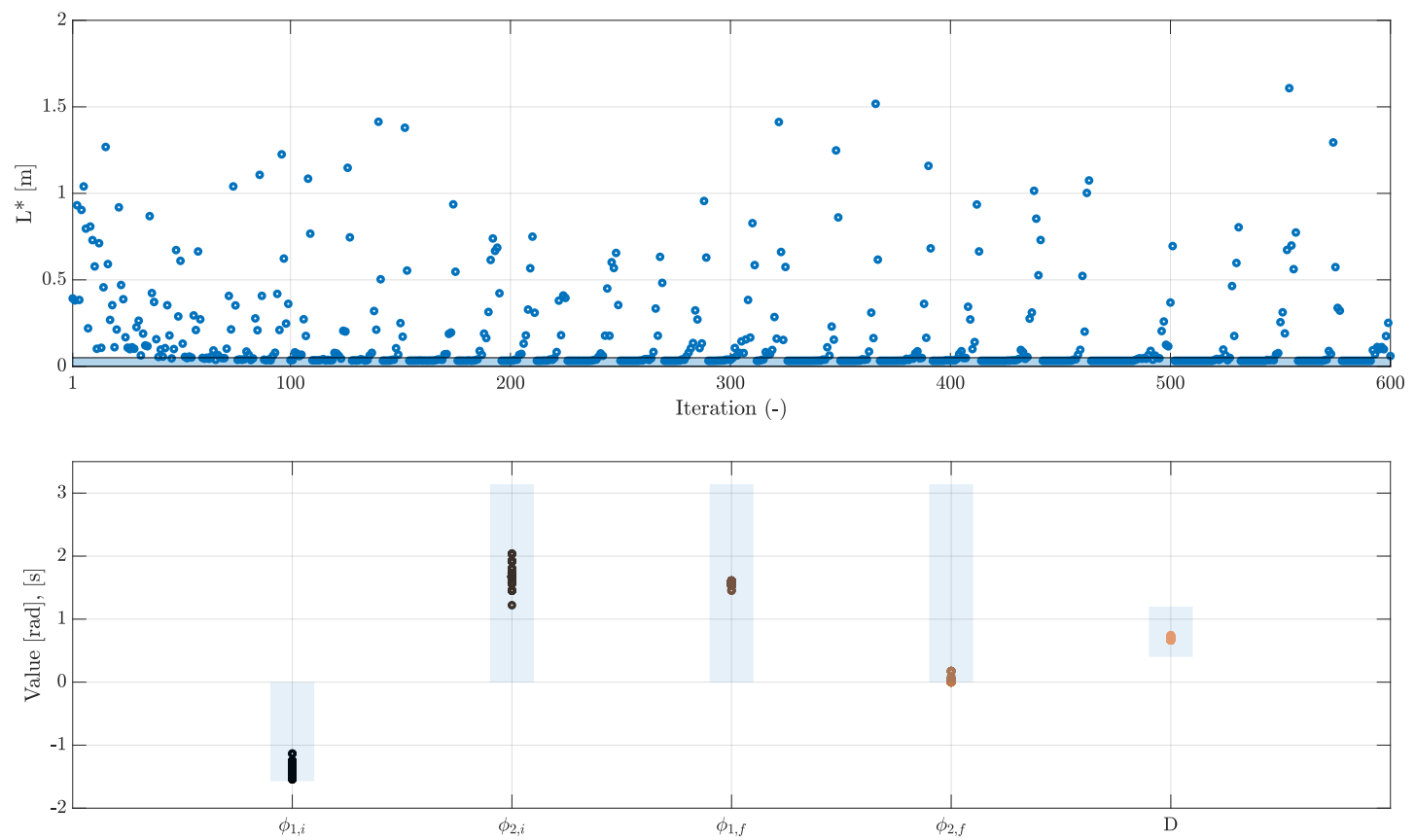

Figure 7-11: (Top) Number of iterations vs. $L^{*}$ value for the short-whip model. The shaded region depicts $L^{*}$ values lower than $0.05 \mathrm{~m}$. (Bottom) Distribution of the data of the corresponding 5 movement parameters, which resulted in $L^{*}$ values lower than $0.05 \mathrm{~m}$. The shaded region depicts the upper and lower bound of the optimization [Table 7.2].

\begin{tabular}{|c|c|c|c|c|c|}
\hline \multirow{2}{*}{ Measures } & \multicolumn{5}{|c|}{ Movement Parameters } \\
\cline { 2 - 6 } & $\phi_{1, i}$ & $\phi_{2, i}$ & $\phi_{1, f}$ & $\phi_{2, f}$ & $D$ \\
\hline \hline Mean & -1.3185 & 1.6798 & 1.5672 & 0.0267 & 0.6751 \\
\hline Std. & 0.0459 & 0.0804 & 0.0174 & 0.0442 & 0.0151 \\
\hline Max & -1.1345 & 2.0362 & 1.6096 & 0.1745 & 0.7404 \\
\hline Min & -1.5417 & 1.2217 & 1.4544 & 0.0001 & 0.6669 \\
\hline C.O.V. & 0.0349 & 0.0479 & 0.0111 & 1.6526 & 0.0224 \\
\hline S.E. & 0.0025 & 0.0044 & 0.0009 & 0.0024 & 0.0008 \\
\hline
\end{tabular}

Table 7.4: The mean, standard deviation (std.), maximum (Max), minimum (Min), coefficient of variation (C.O.V.) and standard error (S.E.) values of the 5 movement parameters which yielded $L^{*}$ values lower than $0.05 \mathrm{~m}$. The number of samples was 339. 


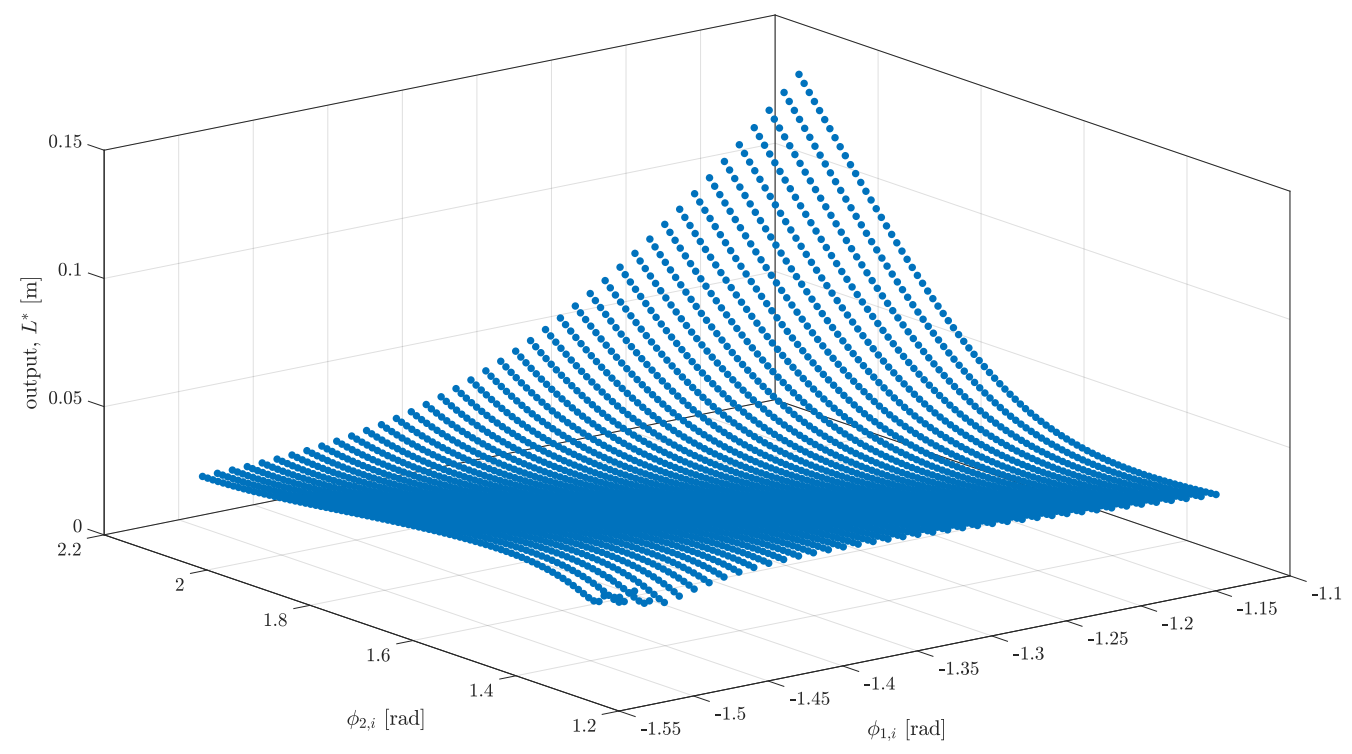

Figure 7-12: 3D plot of the initial shoulder angle $\phi_{1, i}$, initial elbow angle $\phi_{2, i}$ of the zero-torque trajectory vs. output $L^{*}$. The points were evenly sampled with $0.001-$ radian step size for shoulder and elbow angles. In total, 3362 points were sampled.

How could the landscape of output value $L^{*}$ be relatively smooth despite the highly nonlinear dynamics of the whip? It appears that damping of the whip model $b$ may account for this smooth landscape. From the eigenvalue analysis of the linearized $N$ node whip model [Section 5.1], even with a modest amount of damping, it was shown that the high-frequency oscillatory behavior of the damped whip model quickly faded, and the whip was dominated by (eigen)modes with the lowest frequencies. Hence, damping of the whip model "smoothed" the complex whip dynamics, resulting in a less chaotic behavior.

To test this explanation, we examined how the landscape of the objective function, $L^{*}$ changed due to varying the damping value $b$ of the whip. We predicted that as the damping value $b$ decreased, the landscape $L^{*}$ would lose its smoothness and exhibit multiple local minima. That was observed [Figure 7-13].

By gradually decreasing the damping value $b$ to zero, the chaotic dynamics of the whip became more evident. The irregularity of the landscape suggested an increased vulnerability to converging to a local minimum. However, even a modest amount 
of damping, which could be physically justified from experimental results [Chapter 6], was sufficient to "robustify" the optimization by eliminating local minima. This robustness implies that good performance of the whip task could be achieved even with slight deviations from the optimal movement. This may (partially) explain how humans can reduce variability of output performance despite ubiquitous noise which is present at all stages of sensorimotor control. 







\subsection{Optimization of the Experimentally-fitted Whip Model}

Encoding the movement with parameterized dynamic motor primitives succeeded to identify an optimal upper-limb movement. However, all three whip models were based on a single sub-model, the parameters of which were not based on actual measurements. Hence, an optimization of a (more) realistic whip model was additionally conducted. The experimentally-fitted whip model [Table 6.1] was subjected to the exact same optimization process used for the previous three whip models [Section 7.2, 7.3].

As with the previous whip models, the experimentally-fitted whip model also converged to an optimal solution. Detailed values of the optimal parameter set of the discrete movement, $\left(\phi_{1, i}, \phi_{2, i}, \phi_{1, f}, \phi_{2, f}, D\right)$, and its corresponding output $L^{*}$, are presented in [Table 7.5]. Actual movements in simulation generated with the optimal movement parameters are shown in [Figure 7-14], and the graph of the number of iteration vs. $L^{*}$ is shown in [Figure 7-15].

\begin{tabular}{|c|c|c|c|c|c|c|}
\hline \multirow{2}{*}{ Whip Type } & \multicolumn{5}{|c|}{ Optimal Movement Parameters } & Output \\
\cline { 2 - 7 } & $\phi_{1, i}$ & $\phi_{2, i}$ & $\phi_{1, f}$ & $\phi_{2, f}$ & $D$ & $L^{*}$ \\
\hline \hline Exp.-fitted & -1.367 & 0.015 & 1.571 & 0.054 & 0.810 & 0.015 \\
\hline
\end{tabular}

Table 7.5: Optimal movement parameters and the corresponding output value $L^{*}$. Exp.-fitted denotes experimentally-fitted whip model

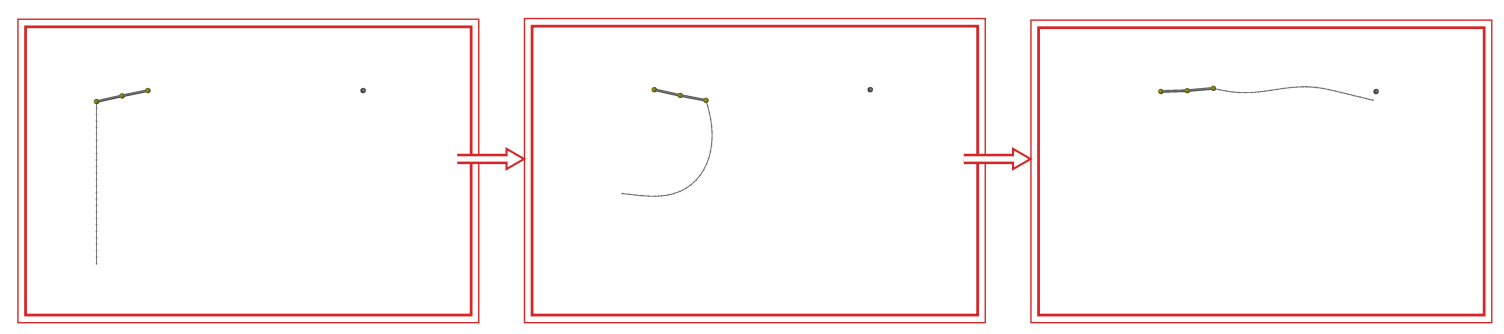

Figure 7-14: Time sequence of the simulation of the experimentally-fitted whip model. Each upper-limb movement was generated with the optimal movement parameters, which yielded the minimum distance $L^{*}$ [Table 7.3]. 
Compared to the short-, medium- and long-whip model, the experimentally-fitted whip model showed the best performance of the whip-targeting task [Table 7.3, 7.5]. The best performance was accomplished even with the highest number of nodes $N$ (25 for the experimentally-fitted whip model which is higher than 20 of the longwhip model), and with a relatively long length of the whip model $(1.8 \mathrm{~m}$ for the experimentally-fitted whip model which is comparable to $2.0 \mathrm{~m}$ of the long-whip model). This result may be due to the quantitative differences of the inertial, stiffness and damping parameters of the model. The total mass of the experimentally-fitted whip model was lowest compared to the others, while the values of stiffness and damping were relatively high [Table 7.3, 7.5]. As a result, the whip dynamics exhibited a much "stiffer" behavior, which may account for why this model resulted in the lowest optimal $L^{*}$ value compared to other whip models. 







\section{Chapter 8}

\section{Spatial Whip Task}

The 2D planar whip task was generalized to 3D. The goal was to reach a distant target with the whip using a single discrete (point-to-point) upper-limb movement. Multiple target positions were tested for the spatial whip task. For all of the MuJoCo simulations, the semi-implicit Euler method was chosen as the numerical integrator, with a time step of $0.1 \mathrm{~ms}$.

\subsection{Modeling}

The discretized $N$-node spatial whip model [Section 3.2] was connected to a 4 degreesof-freedom spatial upper-limb model [Section 3.4]. The handle, which connected the upper-limb and the $N$-node whip model, consisted of 2 freely-rotating hinge joints perpendicular to each other. This connection was established in simulation by setting the torsional stiffness $k$ and damping coefficients $b$ of the sub-model, which directly attached to the upper-limb model, as zero.

Summarizing, the combined 3D model simulated a sequential open-chain mechanism with $2 N+4$ degrees-of-freedom: 4 for the upper-limb model and $2 N$ for the whip model and its handle, a total of $4 N+8$ state variables [Figure 8-1, 8-2]. 


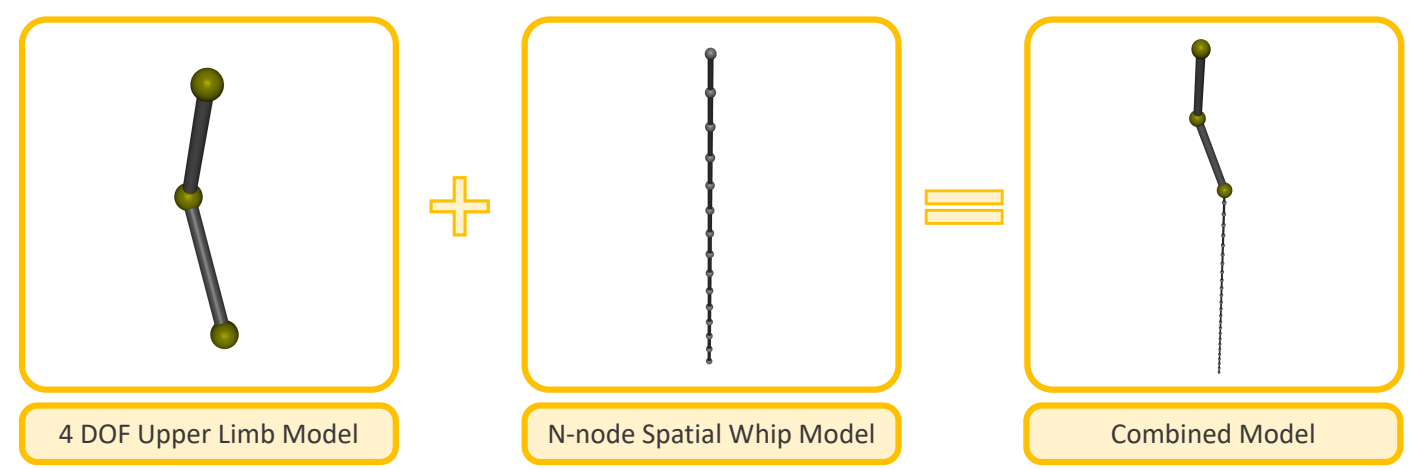

Figure 8-1: The model used for the spatial whip task. A 4 degrees-of-freedom (DOF) upper-limb and spatial whip model were combined.
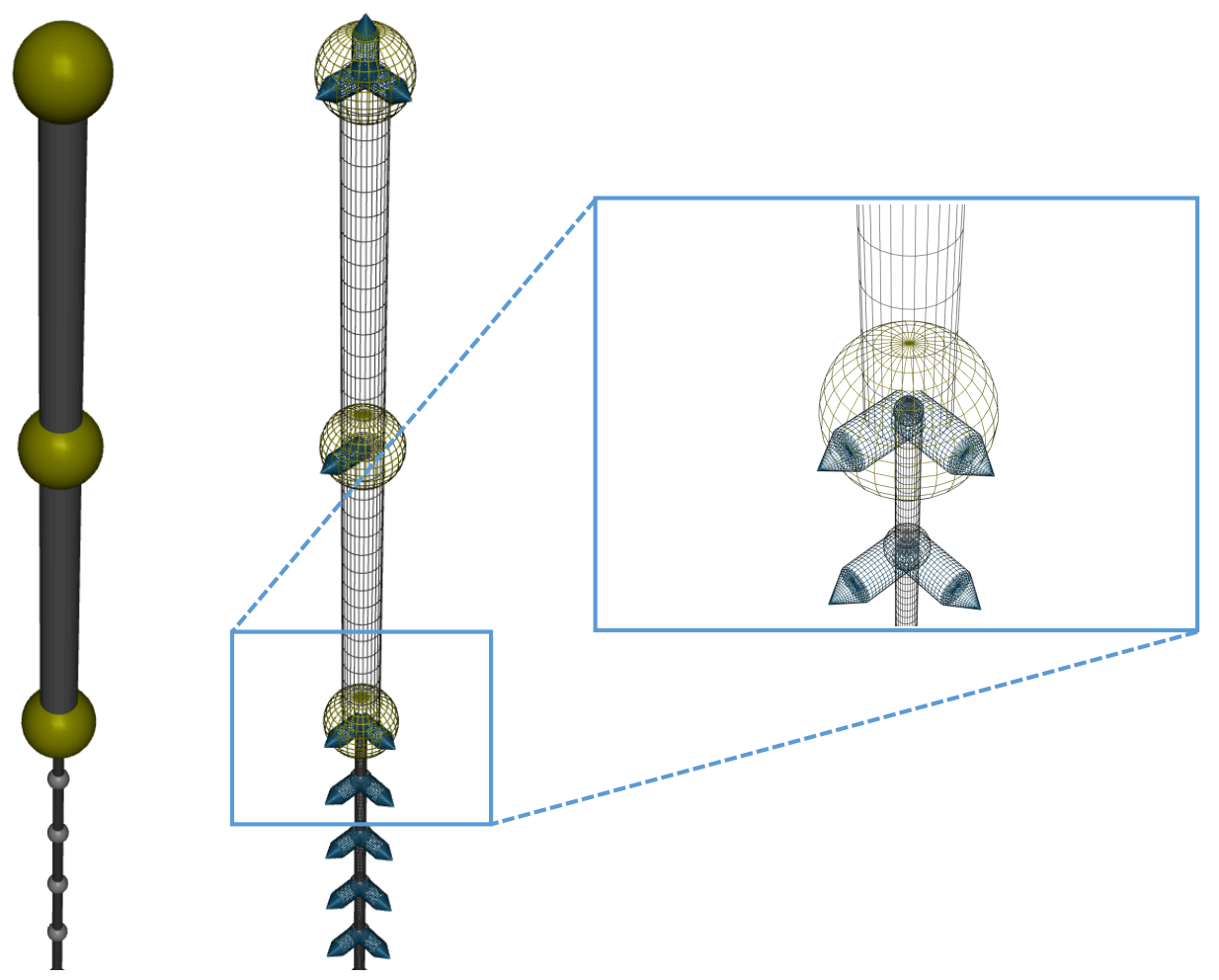

Figure 8-2: The upper-limb and the spatial whip model at an equilibrium posture. The connection of the upper-limb and whip model was achieved by 2 freely-rotating hinge joints perpendicular to each other. Axes of rotation for each joint are visualized as a bullet shapes. 


\subsection{Control of the upper-limb}

\subsubsection{Impedance Controller}

The vector form impedance controller of the planar upper-limb controller [Eqn. 7.1] was used for the spatial upper-limb controller. The only difference was the size of the matrices and vectors of the equation; they were expanded to include the 4 joints of the spatial upper-limb model:

$$
\boldsymbol{\tau}=\boldsymbol{K}(\boldsymbol{\phi}-\boldsymbol{\theta})+\boldsymbol{B}(\dot{\boldsymbol{\phi}}-\dot{\boldsymbol{\theta}})+\boldsymbol{\tau}_{G}
$$

In this equation, $\boldsymbol{K}, \boldsymbol{B} \in \mathbb{R}^{4 \times 4}$ are a constant joint stiffness matrix and a constant joint damping matrix, respectively; vector $\boldsymbol{\tau}(t)=\left[\tau_{1}(t), \tau_{2}(t), \tau_{3}(t), \tau_{4}(t)\right]^{T}$ denotes the net torque input on each joint; vector $\boldsymbol{\tau}_{G}(t)=\left[\tau_{1, G}(t), \tau_{2, G}(t), \tau_{3, G}(t), \tau_{4, G}(t)\right]^{T}$ denotes gravity compensation torque; vector $\boldsymbol{\theta}(t)=\left[\theta_{1}(t), \theta_{2}(t), \theta_{3}(t), \theta_{4}(t)\right]$ denotes the actual joint angle trajectory defined in relative angle coordinates; vector $\phi(t)=\left[\phi_{1}(t), \phi_{2}(t), \phi_{3}(t), \phi_{4}(t)\right]$ represents a motion command from the CNS as a zero-torque trajectory; subscripts 1 to 4 denote joints for shoulder flexion/extension (J1), shoulder adduction/abduction (J2), lateral/medial rotation (J3) and elbow flexion/extension (J4), respectively [Figure 3-4]. Equation [Eqn. 7.3] is used for the gravity compensation torque $\boldsymbol{\tau}_{G}(t)$, with Jacobian matrix $\boldsymbol{J}_{\boldsymbol{i j}} \in \mathbb{R}^{3 \times 4}$ for the spatial upper-limb model.

The details of the 4-by-4 stiffness matrix $\boldsymbol{K}$ and damping matrix $\boldsymbol{B}$ of the spatial upper-limb model were determined based on three key assumptions:

- The shoulder joints J2, J3 (excluding the shoulder flexion/extension joint, J1) and elbow flexion/extension joint J4 were perfectly decoupled.

- The stiffness matrix was constant in relative angle coordinate.

- The damping matrix $\boldsymbol{B}$ was chosen to be proportional to joint stiffness $\boldsymbol{K}$, such that $\boldsymbol{B}=\beta \boldsymbol{K}$, where $\beta$ was a time-constant of $0.05 \mathrm{~s}$, such that $\boldsymbol{B}=0.05 \boldsymbol{K}$ 
Experimental measurements of shoulder impedance were used to determine the 4by-4 neuromuscular stiffness matrix $\boldsymbol{K}$. For the 3-by-3 stiffness submatrix which corresponded to the three shoulder joints (J1, J2 and J3), experimentally measured values were used [Lipps et al., 2015]. For the 2-by-2 stiffness submatrix which corresponded to the shoulder flexion/extension joint (J1) and elbow flexion/extension joint (J4), the stiffness matrix of the planar upper-limb model was used [Eqn. 7.2]. However, that 2 -by-2 matrix was multiplied by a scaling constant (valued as $17.4 / 29.5 \approx 0.590$ ), so that the diagonal element of the stiffness matrix for joint J1 matched the experimentally measured value reported by [Lipps et al., 2015].

The resulting $\boldsymbol{K}$ and $\boldsymbol{B}$ matrices were as follows:

$$
\boldsymbol{K}=\left[\begin{array}{cccc}
17.4 & 4.7 & -1.9 & 8.4 \\
9.0 & 33.0 & 4.4 & 0.0 \\
-13.6 & 3.0 & 27.7 & 0.0 \\
8.4 & 0.0 & 0.0 & 23.2
\end{array}\right], \quad \boldsymbol{B}=\left[\begin{array}{cccc}
0.87 & 0.235 & -0.095 & 0.42 \\
0.45 & 1.650 & 0.22 & 0.0 \\
-0.68 & 0.150 & 1.385 & 0.0 \\
0.42 & 0.0 & 0.0 & 1.16
\end{array}\right]
$$

Note that the stiffness and damping matrices of the spatial upper-limb model were non-symmetric, compared to the planar upper-limb model where the $\boldsymbol{K}$ and $\boldsymbol{B}$ matrices were symmetric. This non-symmetric property implied that the stiffness matrix $\boldsymbol{K}$ and damping matrix $\boldsymbol{B}$ had a skew-symmetric component, which indicated a non-zero curl element.

\subsubsection{Zero-Torque Trajectory}

The zero-torque trajectory of the spatial upper-limb model followed a rest-to-rest minimum-jerk profile in joint coordinates:

$$
\phi(t)=\phi_{i}+\left(\phi_{f}-\phi_{i}\right) \cdot\left\{10\left(\frac{t}{D}\right)^{3}-15\left(\frac{t}{D}\right)^{4}+6\left(\frac{t}{D}\right)^{5}\right\}
$$


where $t$ is time and subscripts $i$ and $f$ denote the initial and final postures, respectively. For times greater than the duration $D$ (i.e. $t>D$ ), the zero-torque trajec-

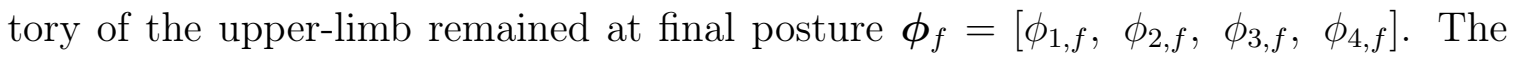
zero-torque trajectory $\phi(t)$ was determined by 9 movement parameters: 4 for initial posture $\phi_{i}, 4$ for final posture $\phi_{f}$, and 1 for the movement duration $D$.

\subsection{Optimization Method}

The objective of the spatial whip task was to minimize the value $L[m]$, the distance between the tip of the whip and a target with a single discrete spatial upper-limb movement, i.e. a single set of 9 movement parameters $\left(\phi_{i}, \phi_{f}, D\right)$. The minimum value of the distance $L$ reached with a single discrete (i.e. rest-to-rest) upper-limb movement, $L^{*}(m)$, was a quantitative measure to assess the performance of the spatial whip task. The objective of the whip task was cast as an optimization problem: finding the optimal 9 movement parameters which resulted in the minimum $L^{*}$ value.

The DIRECT-L algorithm was used for the optimization. The bounding box constraint for the 9 movement parameters are presented in [Table 8.1]. Within the bounds of the constraint, the DIRECT-L optimization algorithm conducted 600 iterations.

\begin{tabular}{|c|c|c|c|c|c|c|c|c|c|}
\hline & $\phi_{1, i}$ & $\phi_{2, i}$ & $\phi_{3, i}$ & $\phi_{4, i}$ & $\phi_{1, f}$ & $\phi_{2, f}$ & $\phi_{3, f}$ & $\phi_{4, f}$ & $D$ \\
\hline \hline Low. B. & $-0.5 \pi$ & $-0.5 \pi$ & $-0.5 \pi$ & 0 & $0.1 \pi$ & $-0.5 \pi$ & $-0.5 \pi$ & 0 & 0.4 \\
\hline Up. B. & $-0.1 \pi$ & $0.5 \pi$ & $0.5 \pi$ & $0.9 \pi$ & $1.0 \pi$ & $0.5 \pi$ & $0.5 \pi$ & $0.9 \pi$ & 1.5 \\
\hline
\end{tabular}

Table 8.1: Lower bound (Low. B.) and upper bound (Up. B.) of the 9 movement parameters used for the DIRECT-L optimization algorithm.

The spatial version of the experimentally-fitted whip model was used for the spatial whip task [Table 7.1], i.e. since $N=25$, the whole model simulated a sequential open-chain mechanism with 54 degrees-of-freedom [Section 8.1]. Three different target locations were defined for the spatial whip task. All three targets were distanced just $0.01 \mathrm{~m}$ outside of a sphere, centered at the shoulder joint, of radius $R[\mathrm{~m}]$ equal to the sum of the lengths of the upper-limb and the length of the experimentally-fitted 
whip model $(R=L 1+L 2+1.8+0.01=2.395 \mathrm{~m})$ [Table 3.1].

In a spherical coordinate system (radius-azimuth-elevation), target 1 was located at coordinate $\left(R, 0^{\circ}, 0^{\circ}\right)$; target 2 was located at coordinate $\left(R, 45^{\circ}, 0^{\circ}\right)$; target 3 was located at coordinate $\left(R, 45^{\circ}, 45^{\circ}\right)$ [Figure 8-3]. Optimization of upper-limb movements for each target were performed.

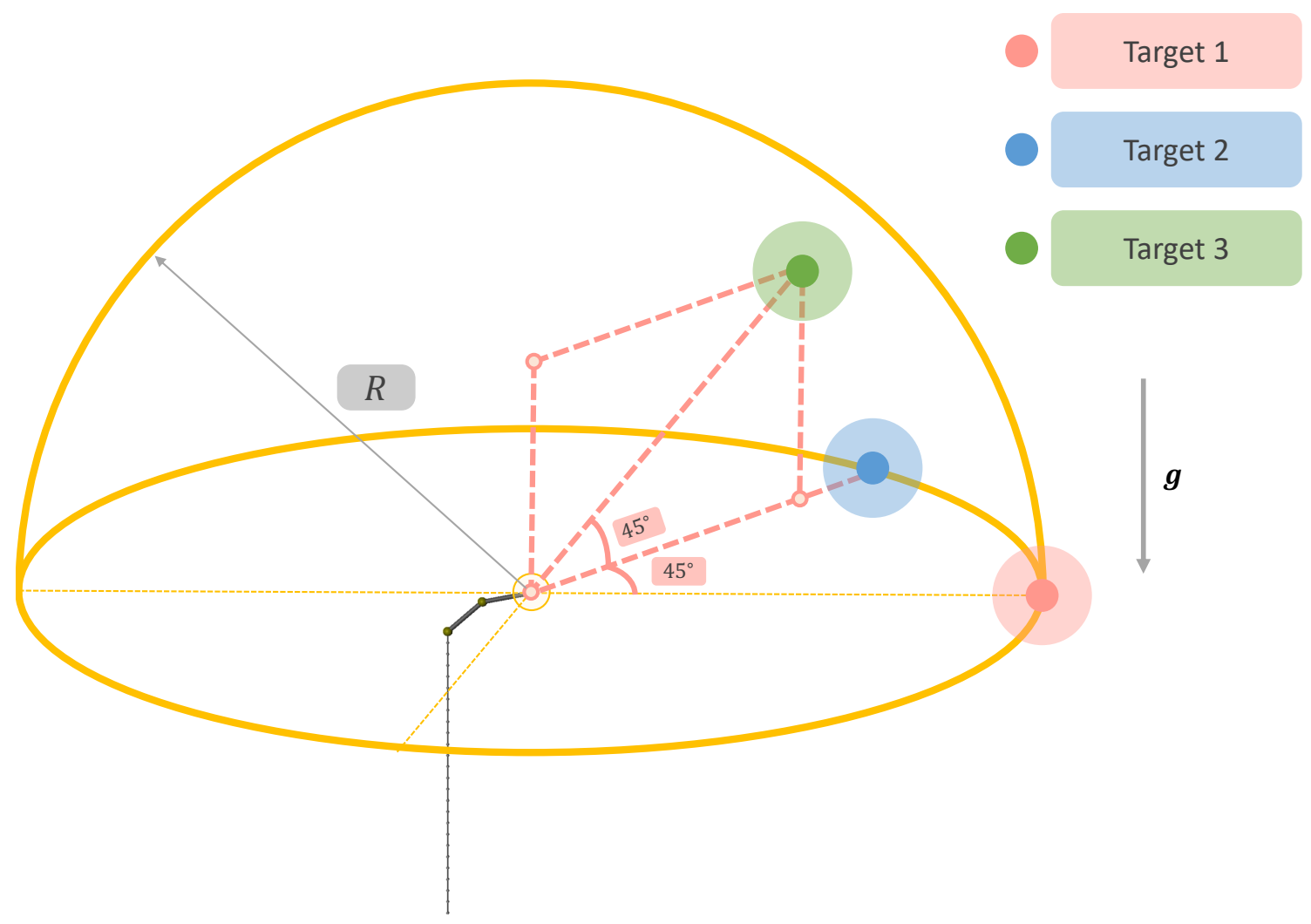

Figure 8-3: The target positions depicted in a spherical coordinate system. $R[\mathrm{~m}]$ denote the radius of the sphere.

\subsection{Optimization and Simulation Results}

For each target position, the DIRECT-L algorithm converged to an optimal set of 9 movement parameters which yielded the minimum value of distance $L^{*}$ for the experimentally-fitted whip model. Detailed values of the optimal parameter set of the motion, $\left(\phi_{i}, \phi_{f}, D\right)$, and the corresponding output $L^{*}$, are presented in [Table 8.2]. A time-lapse of the simulation generated with the optimal movement parameters 
is shown in [Figure 8-4, 8-5, 8-6]. The trajectories of the optimal 3D upper-limb movement (excluding the whip) are emphasized and separately plotted in [Figure 8-7, 8-8, 8-9]. Images were produced using MATLAB based on the position data extracted from the MuJoCo simulation. Even though the whip task was generalized from 2D to 3D, the optimization converged to an optimal spatial upper-limb movement.

Target 1 was at exactly the same location as the planar whip task. The only difference was the dimensionality of the movement: the planar whip task was constrained to the 2D sagittal plane; the spatial whip task used the whole $4 \mathrm{D}$ joint space. Minor differences of performance were observed between the planar and spatial whip task. For the experimentally-fitted whip model, the optimal $L^{*}$ values were $0.015 \mathrm{~m}$ and $0.050 \mathrm{~m}$ for the planar and spatial whip task, respectively [Table 7.5, 8.2]. The slightly larger value of $L^{*}$ for the spatial whip task may be due to the higher degrees-of-freedom of each node for the spatial whip model - each node of the spatial whip model had two degrees-of-freedom, while the planar whip model had a single degree-of-freedom.

For reaching target 1 with the whip model, the contribution of shoulder joints J2 and J3 were relatively small compared to the others. Shoulder joint J2 remained exactly at zero for the whole movement: $\phi_{i, 2}$ and $\phi_{f, 2}$ were zero. Shoulder joint J3 started at a moderate value, $\phi_{3, i}=0.349[\mathrm{rad}]$, and ended at zero, $\phi_{3, f}=0[\mathrm{rad}]$. Considering that joints J1 and J4 respectively corresponded to the shoulder and elbow joints of the planar upper-limb model, it can be concluded that the optimal spatial upper-limb movement for target 1 resembled that computed for the planar case. This result is clearly shown in the $x y$-plane view of the optimal movement [Figure 8-4, 8-7], where the optimal spatial upper-limb movement was approximately planar and mostly confined to the $2 \mathrm{D}$ sagittal plane.

The task of reaching target 2 was simply a rotated version of target 1 . Considering the rotational symmetry of this model of the whip task, the difficulty of reaching target 2 was equivalent to the task of reaching target 1 . However, even though the optimal $L^{*}$ value for target 1 and target 2 were similar $(0.050 \mathrm{~m}$ and $0.069 \mathrm{~m}$, respectively), the optimal movement for target 2 provided from the optimization algorithm showed a 
substantial difference from the optimal movement for target 1 [Figure 8-7, 8-8]. Rather than the movement being fully-stretched with a large planar "sweeping" motion, the optimal movement for target 2 was "spiraled" and non-planar.

The optimal value of $L^{*}$ for target 3 was the largest compared to the others. The optimal upper-limb movement for target 3 resembled the spiraled, non-planar optimal movement of target 2 [Figure 8-5, 8-6, 8-8, 8-9].

\begin{tabular}{|c|c|c|c|}
\hline \multirow{2}{*}{ Parameters } & \multicolumn{3}{|c|}{ Target Type } \\
\cline { 2 - 4 } & Target 1 & Target 2 & Target 3 \\
\hline \hline$\phi_{i, 1}$ & -1.554420 & -1.361357 & -1.361357 \\
\hline$\phi_{i, 2}$ & 0.000000 & 1.047198 & 0.349066 \\
\hline$\phi_{i, 3}$ & -0.349066 & -0.297352 & -0.698132 \\
\hline$\phi_{i, 4}$ & 1.378810 & 1.111193 & 2.042035 \\
\hline$\phi_{f, 1}$ & 1.727876 & 1.727876 & 1.727877 \\
\hline$\phi_{f, 2}$ & 0.000000 & -1.034269 & -1.047198 \\
\hline$\phi_{f, 3}$ & 0.000000 & -0.754155 & -0.349066 \\
\hline$\phi_{f, 4}$ & 0.351005 & 0.157080 & 0.471239 \\
\hline$D$ & 0.950000 & 0.950000 & 0.583333 \\
\hline \hline$L^{*}$ & 0.050384 & 0.069136 & 0.162527 \\
\hline
\end{tabular}

Table 8.2: Optimal movement parameters and the corresponding output value $L^{*}$ for each target. 


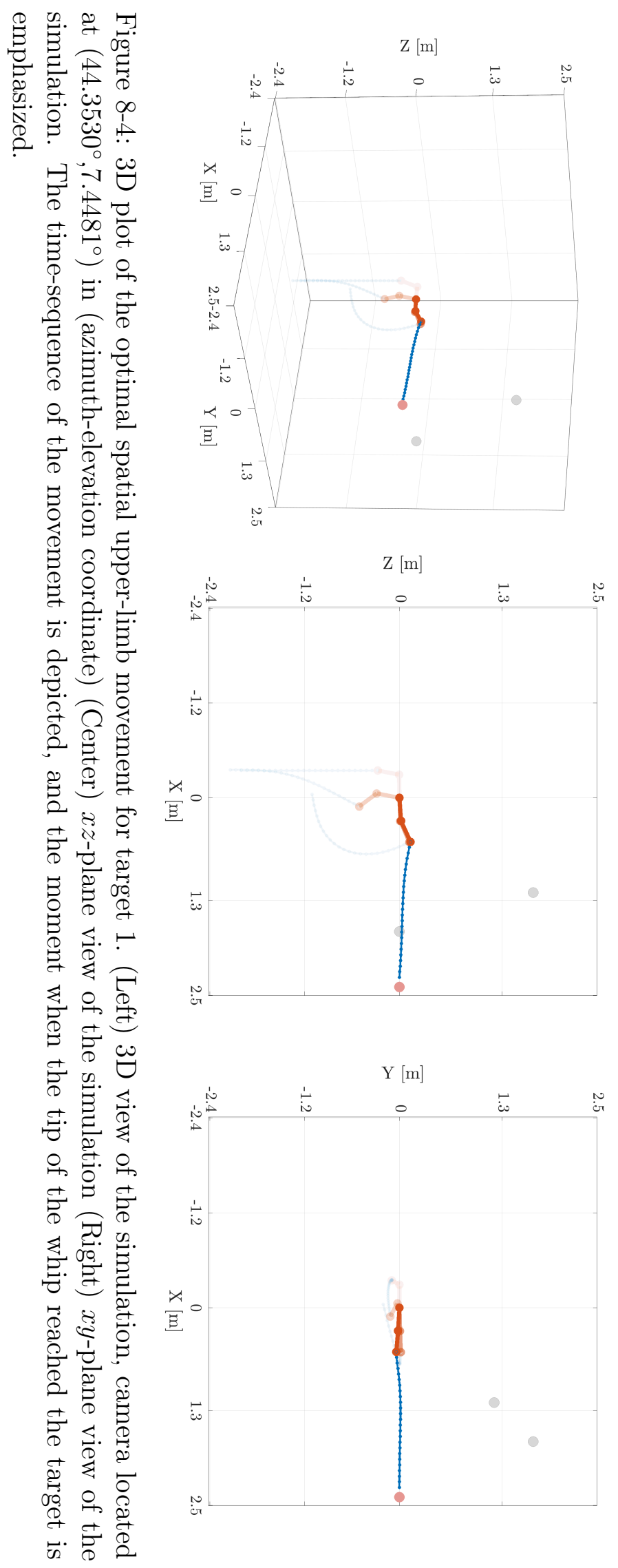




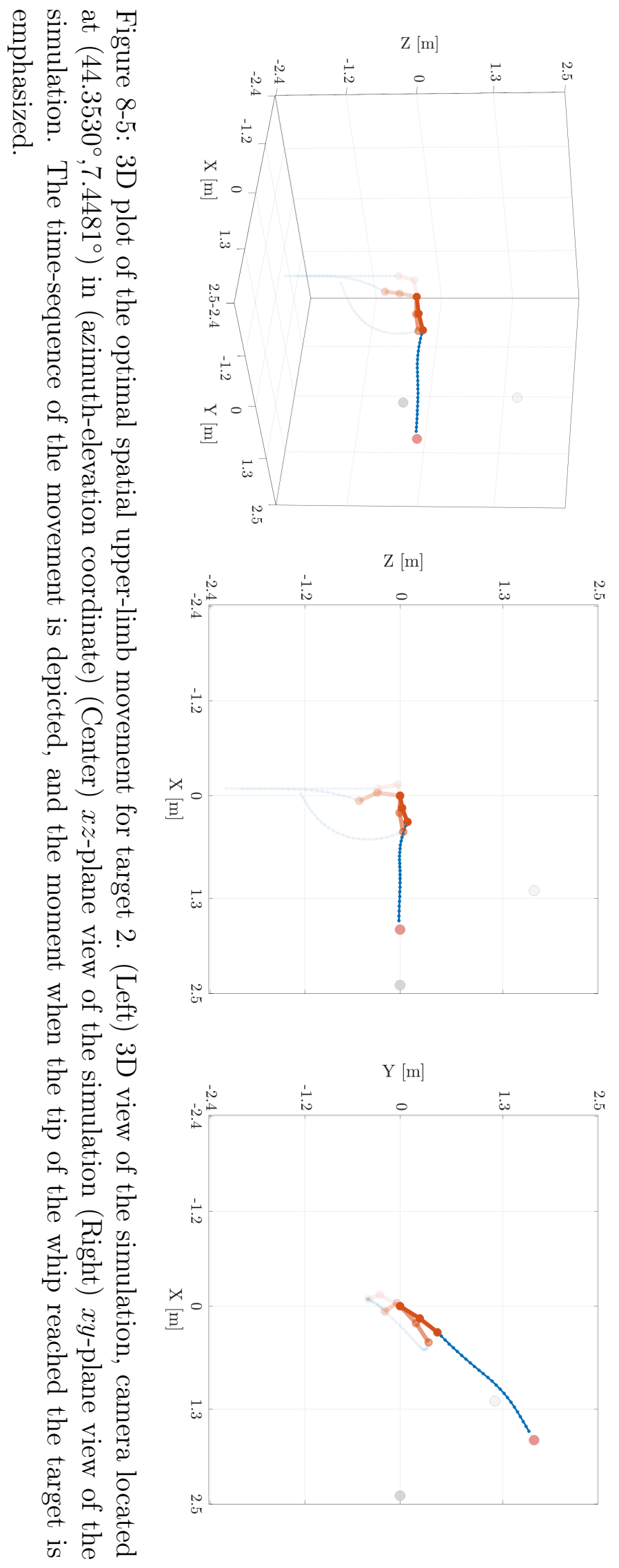




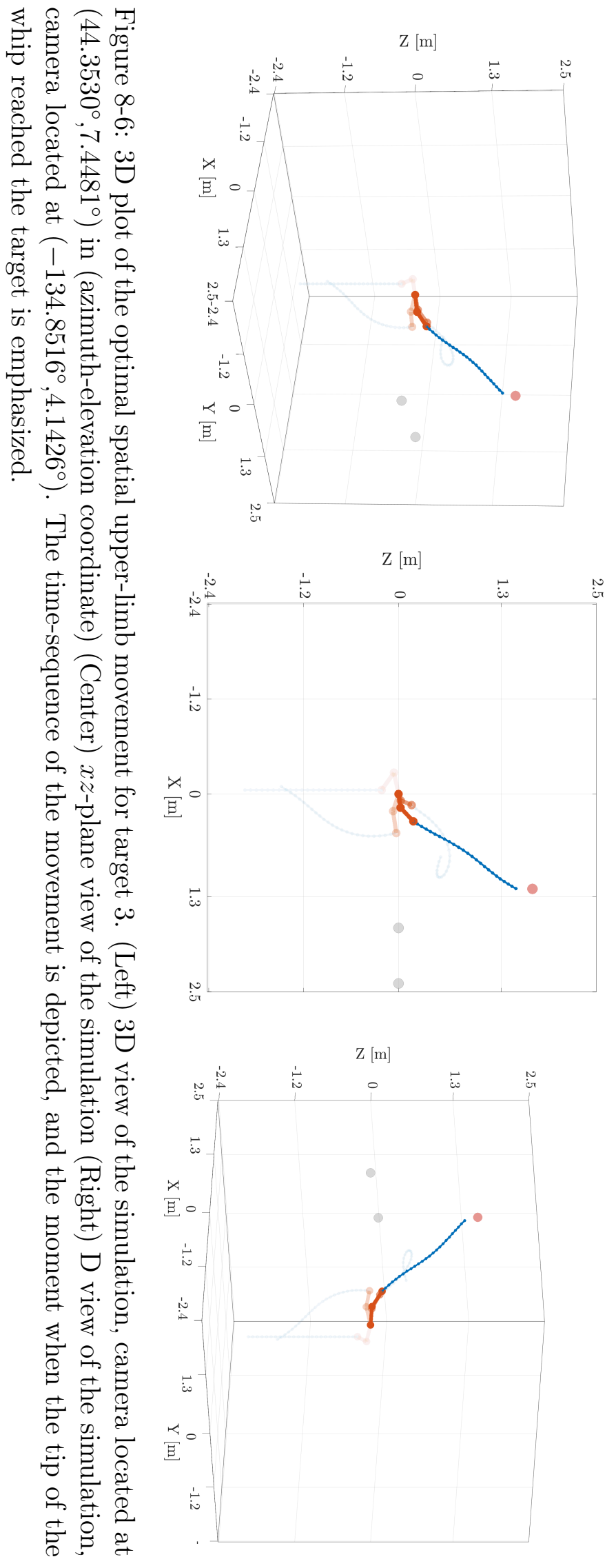




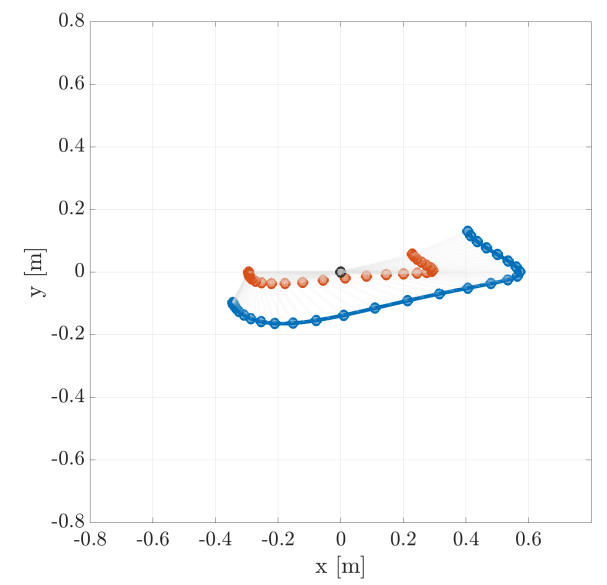

(a) $x y$-plane view.

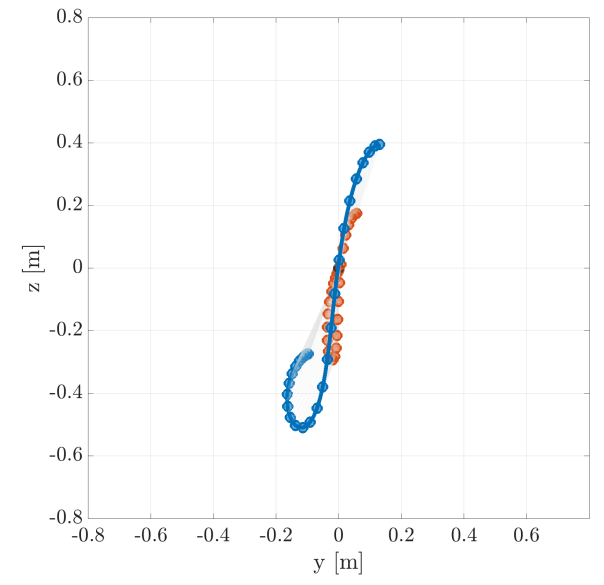

(c) yz-plane view.

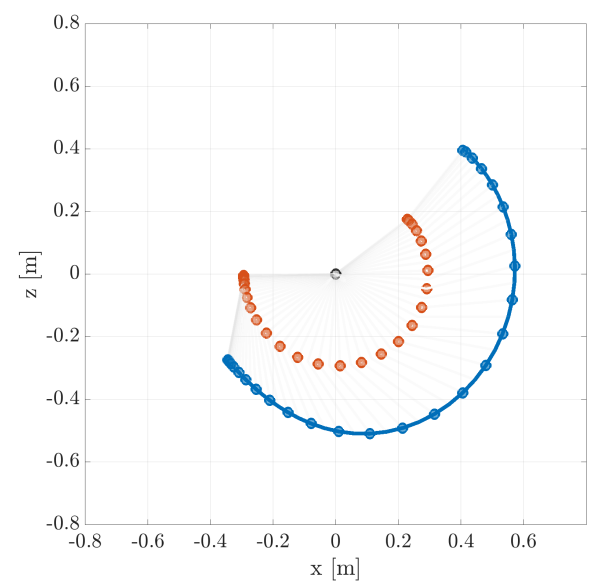

(b) $x z$-plane view.

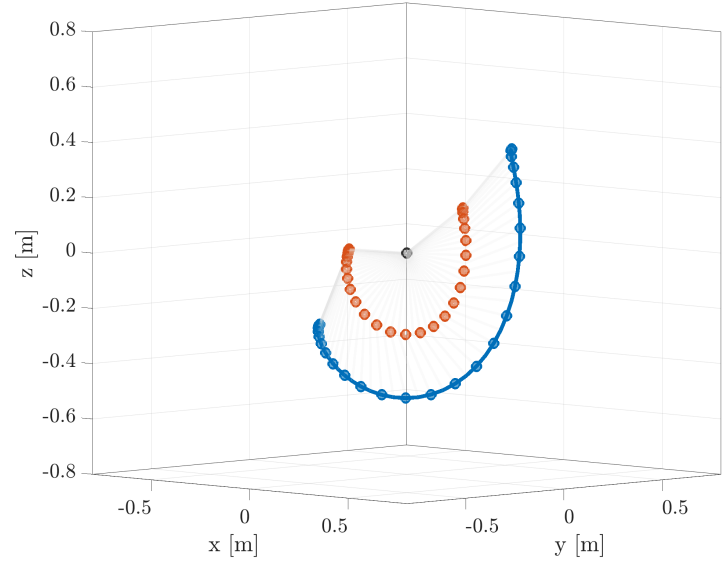

(d) 3D view

Figure 8-7: 3D plot of the optimal upper-limb movement for target 1. Shoulder joint trajectory is colored in orange, and the end-point trajectory of the upper-limb is colored in blue. 


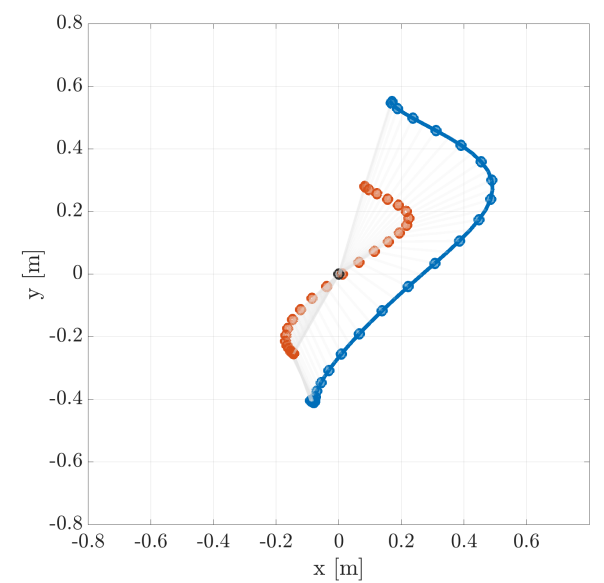

(a) $x y$-plane view

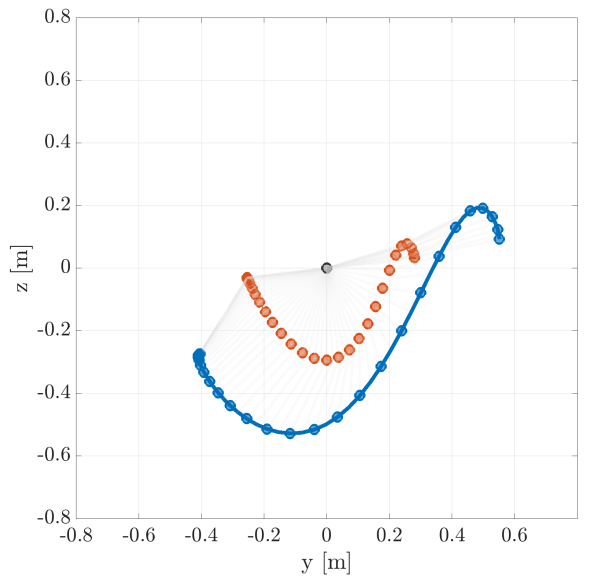

(c) $y z$-plane view

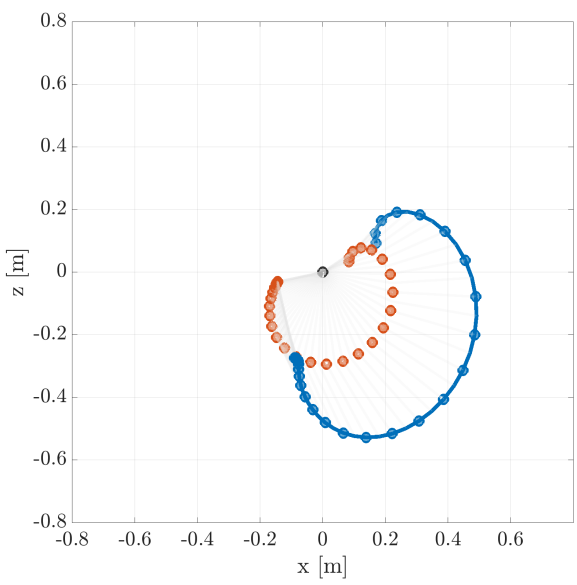

(b) $x z$-plane view

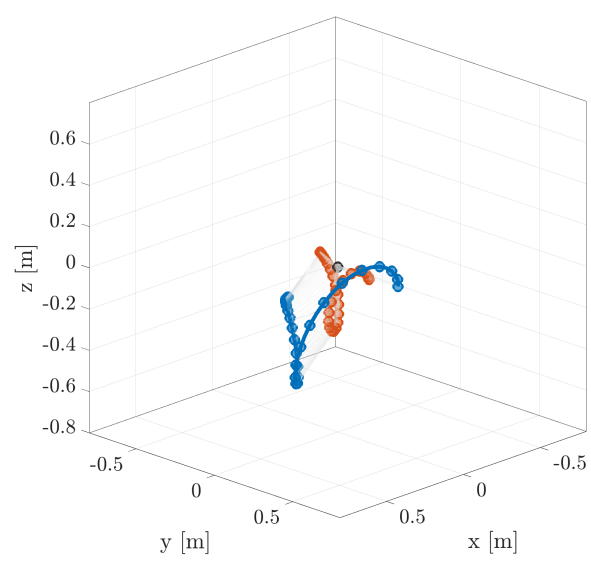

(d) 3D view

Figure 8-8: 3D plot of the optimal upper-limb movement for target 2. Shoulder joint trajectory is colored in orange, and the end-point trajectory of the upper-limb is colored in blue. 


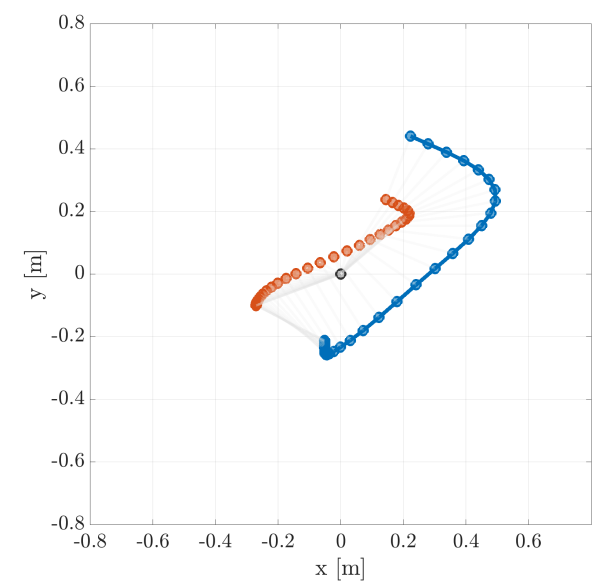

(a) $x y$-plane view

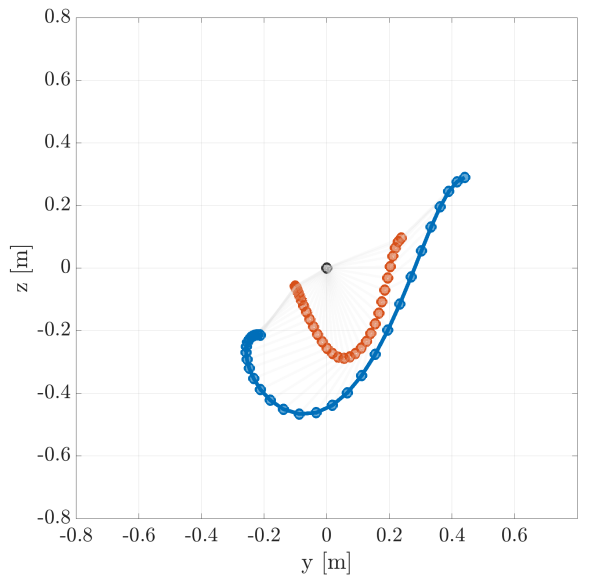

(c) $y z$-plane view

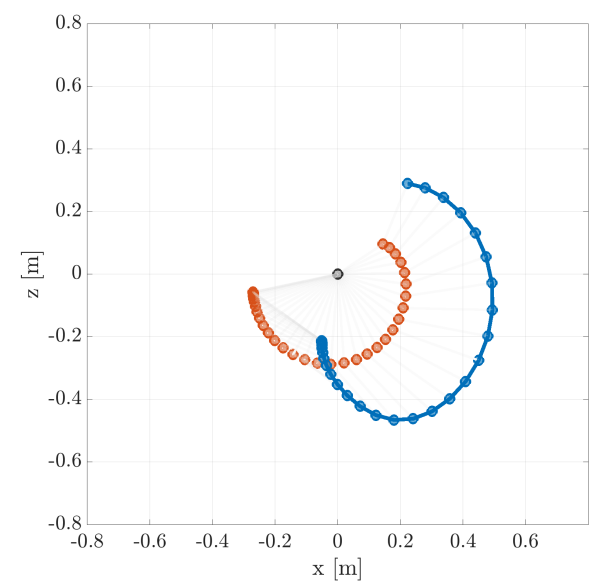

(b) $x z$-plane view



(d) $3 \mathrm{D}$ view

Figure 8-9: 3D plot of the optimal upper-limb movement for target 3. Shoulder joint trajectory is colored in orange, and the end-point trajectory of the upper-limb is colored in blue. 


\section{Chapter 9}

\section{Conclusions and Discussion}

This study examined whether a target could be reached with a simulated whip using a (small) number of dynamic primitives, whose parameters could be learned through optimization. Because of the dynamic complexity of the whip model, this simple task is anything but trivial. It was not a priori obvious that the optimization would even converge, let alone produce a meaningful result.

By encoding upper-limb action using the parameters of dynamic primitives, the acquisition of the motor skill to achieve this task was greatly simplified. Simplifying the motor task via parameterized dynamic primitives dramatically reduced the computational complexity of the optimization problem, providing a way to work-around the "curse of dimensionality". This approach successfully managed the complexity of an (extremely) high degree-of-freedom system: for 2D, the highest was a 27 degreesof-freedom model, a $54^{\text {th }}$ dimensional state-space representation; for 3D, the highest was a 54 degrees-of-freedom model, a $108^{\text {th }}$ dimensional state-space representation. In all cases, a minimum distance from the target was achieved with a single discrete movement.

It is worth emphasizing that the upper-limb controller was "ignorant" of the complex whip dynamics. While it is straightforward (albeit tedious) to derive the equations of motion for the sequential chain model for both planar and spatial cases, optimization using such a complex mathematical model seems impractical, challenging even with modern computational resources. Using dynamic motor primitives, 
the whip-targeting task was achieved without the need to store or recall any detailed mathematical representation of the whole system dynamics. This may be a key simplification humans use to learn complex motor skills, since only a small set of parameters may need to be acquired and retained regardless of the dimensionality of the object being manipulated. To the extent that dynamic motor primitives offer a simplified solution to complex and flexible object manipulation, this approach may facilitate robotic manipulation of flexible materials, which is presently a major challenge.

\subsection{Relation to Prior Work}

The movement primitives described by Ijspeert et al. [Ijspeert et al., 2002] have successfully been used in robotics with different applications. Schaal et al. used movement primitives to learn from a demonstrated trajectory [Schaal et al., 2003]. Peters and Schaal showed that a robotic arm was able to learn a baseball swing via movement primitives [Peters and Schaal, 2006]. Stulp et al. used a reinforcement learning algorithm and motion primitives to improve the robustness of grasping and pick-and-place tasks [Stulp et al., 2012].

This prior work mainly focused on comparatively simple objects with few degreesof-freedom, which did not fully account for the true complexity of the range of objects that humans can handle. To the best of our knowledge, using dynamic motor primitives for controlling high degree-of-freedom systems has not previously been studied. Flexible objects with highly complex internal dynamics remain particularly challenging for robots.

The only comparable study we are aware of demonstrated whip-cracking with a robot manipulator [Yamakawa et al., 2016]. However, in that work the motion trajectory of the robot was determined by replicating experimental observations of actual human performance. Planning using optimization based on dynamic motor primitives was not considered. 


\subsection{Limitations and Future Direction}

For the 2D planar whip task, it was notable that the minimum distance became greater as the length of the whip increased. This suggested that a single discrete movement might not be sufficient for longer whip models. Adding a second dynamic primitive may improve performance. In fact, observations of a skilled human performer indicated that one action (sometimes rhythmic) was used to "energize" the whip while a second action (e.g., a "wrist flick") served to propagate a wave along it [Henrot, 2016].

In the work reported here, a single discrete movement with a minimum-jerktrajectory was sufficient to accomplish the targeting task, but different types of movement may be explored. For instance, superimposing oscillations with discrete movements may result in a richer repertoire of upper-limb movements to achieve the task.

The neuromuscular mechanical impedance of the upper-limb model was chosen to be constant, and motion primitives with no consideration of interaction were sufficient to perform the whip-targeting task successfully. For future work, the importance of physical interaction between the upper-limb and whip model will be explored. Specifically, it is speculated that there exists an optimal impedance value of the upper limb which determines the ability to approximate a wave-like behavior of the whole model. If the upper-limb model is too stiff, wave-like behavior may be suppressed. If the upper-limb model is too compliant, the injection of wave energy into the whip may be compromised. We anticipate that a condition resembling "impedance-matching" will be optimal to propagate a wave-like behavior. A series of simulation experiments to test this speculation is a subject of future work.

Discretizing the continuous whip dynamics with a finite number of lumped parameter sub-models enabled a compromise between ease of analysis and fidelity of reproducing essential behavior. However, to avoid cluttering the simulation model with excessive details, some physical properties of the whip (which might be essential) were not considered. For instance, studies reported that the tapering of the whip is crucial 
to achieve whip cracking and wave propagation [Goriely and McMillen, 2002]. Taking these properties into account may be crucial to represent a more realistic model of the whip.

The results reported here do not preclude alternative approaches to reducing the dimensionality of an optimization problem. For example, an input time-history (e.g., of joint torques) might be defined by a sparse number of "knot" points connected by some suitable spline function, and that may also facilitate convergence of the optimization. In essence, the discrete motion profile used here is an extreme example of that approach, using only two knot points for the entire trajectory. However, it is important that the choice of motion profile used was not arbitrary, but based on the highly-stereotyped motion profiles of point-to-point reaching movements made by unimpaired humans; found in the earliest movements made by persons recovering after stroke; and made by infants learning to coordinate their limbs [Hogan, 1982, Flash and Hogan, 1985, von Hofsten, 1991, Berthier, 1996, Rohrer et al., 2004].

In this simulation study the wrist joint was neglected for simplicity, since it is always better to start simple and slowly add complexity, rather than the other way around. Anecdotally, the wrist appears to play a prominent role in skilled whip manipulation. However, at this time the role of the wrist remains unclear. Future work may clarify the role of the wrist.

\subsection{Conclusion}

Despite the significant limitations of our neuromuscular system, humans manipulate objects of prodigious dynamic complexity with apparent ease. The simulations presented here showed that encoding control via the parameters of primitive dynamic actions - discrete movements - enabled optimization to identify actions that managed an extremely complex dynamic object - a whip. Understanding how this is accomplished may facilitate endowing robots with comparable dexterity. 


\section{Appendix A}

\section{Dynamics of the Multi-Pendulum}

\section{A.1 Equation of Motion of the Multi-Pendulum}

By treating the object as a set of "lumped-parameter" sub-models, a continuous infinite-dimensional model can be simplified to a discrete finite-dimensional model.

The continuous dynamics of flexible objects was discretized to a planar $N$-node multi-pendulum model. The planar $N$-node multi-pendulum model consisted of an inextensible massless cylinder, suspended with length $l$ from a single degree-of-freedom pivot with a (ideal) point mass $m$ attached to the other end. The pivot, a rotation joint, was equipped with a linear torsional spring $k$ and linear torsional damper $b$. The lumped-parameters of the $i^{\text {th }}$ pendulum were denoted as $m_{i}, l_{i}, k_{i}$ and $b_{i}$, respectively [Figure A-1]. The multi-pendulum model was suspended from a fixed point in space. 

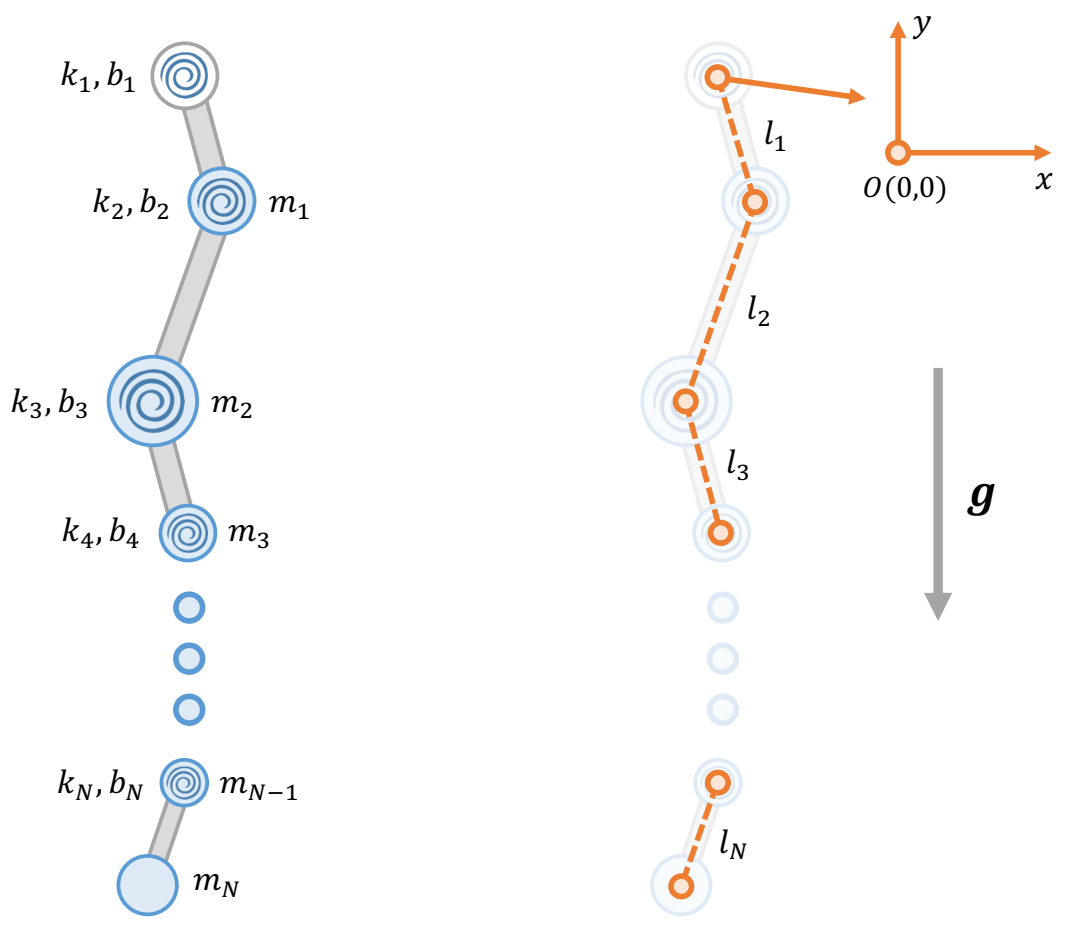

Figure A-1: The $N$-node multi-pendulum model. The Cartesian coordinate frame with its origin $O$ is indicated at the top of the pendulum.

The equation of motion of the $N$-node multi-pendulum model can be derived from the Euler-Lagrange equation. The Lagrangian $L$, a scalar value function, is defined as:

$$
L:=T-V
$$

where $T$ and $V$ denote the total kinetic energy and potential energy of the system, respectively.

In Cartesian coordinates, the kinetic and potential energy can be written as:

$$
T=\frac{1}{2} \sum_{i=1}^{N} m_{i}\left(\dot{x}_{i}^{2}+\dot{y}_{i}^{2}\right), \quad V=g \sum_{i=1}^{N} m_{i} y_{i}
$$

where

$$
x_{i}=\sum_{j=1}^{i} l_{j} \sin \theta_{j}, \quad y_{i}=-\sum_{j=1}^{i} l_{j} \cos \theta_{j}
$$

Dots denote derivatives with respect to time, and angle $\theta_{j}$ is the absolute angle of the 
$j^{\text {th }}$ pendulum measured from the vertically-downward equilibrium posture, i.e. the angle measured from the $-y$ axis of the Cartesian frame [Figure A-1].

The kinetic energy $T$ and potential energy $V$ can be rewritten as a function of absolute angles of the $N$ pendulums. The $N$ absolute angles of the $N$ pendulums were chosen to be the independent generalized coordinate vector $\boldsymbol{\theta}(t)$ :

$$
\boldsymbol{\theta}(t)=\left[\theta_{1}, \theta_{2}, \theta_{3}, \cdots, \theta_{N}\right]
$$

Based on this generalized coordinate vector $\boldsymbol{\theta}(t)$, it was able to derive the $N$ equations of motion of the multi-pendulum model with the Lagrange's equation:

$$
\frac{d}{d t}\left(\frac{\partial L}{\partial \dot{\theta}_{j}}\right)-\frac{\partial L}{\partial \theta_{j}}=Q_{j}
$$

where $Q_{j}$ denotes the generalized force, which was the torque from the linear torsional spring and linear torsional damper. ${ }^{1}$

In Cartesian coordinates, the velocity and acceleration of each point masses are given as:

$$
\begin{array}{ll}
\dot{x}_{i}=\sum_{j=1}^{i} l_{j} \dot{\theta}_{j} \cos \theta_{j}, & \dot{y}_{i}=\sum_{j=1}^{i} l_{j} \dot{\theta}_{j} \sin \theta_{j} \\
\ddot{x}_{i}=\sum_{j=1}^{i} l_{j}\left(\ddot{\theta}_{j} \cos \theta_{j}-\dot{\theta}_{j}^{2} \sin \theta_{j}\right), & \ddot{y}_{i}=\sum_{j=1}^{i} l_{j}\left(\ddot{\theta}_{j} \sin \theta_{j}+\dot{\theta}_{j}^{2} \cos \theta_{j}\right)
\end{array}
$$

The partial derivative of the position and velocity terms with respect to the joint angle $\theta$ are:

$$
\begin{array}{ll}
\frac{\partial x_{j}}{\partial \theta_{i}}=h_{i j} l_{i} \dot{\theta}_{i} \cos \theta_{i} & \frac{\partial y_{j}}{\partial \theta_{i}}=h_{i j} l_{i} \dot{\theta}_{i} \sin \theta_{i} \\
\frac{\partial \dot{x}_{j}}{\partial \theta_{i}}=-h_{i j} l_{i} \dot{\theta}_{i} \sin \theta_{i} & \frac{\partial \dot{y}_{j}}{\partial \theta_{i}}=h_{i j} l_{i} \dot{\theta}_{i} \cos \theta_{i}
\end{array}
$$

\footnotetext{
${ }^{1}$ Strictly speaking, the elastic potential energy due to torsional spring should also be added into the potential energy term $V$, However, for the sake of simplifying the derivation, the effect of torsional spring was added to the generalized force $Q$.
} 
where

$$
h_{i j}= \begin{cases}1 & \text { if } i \leq j \\ 0 & \text { else }\end{cases}
$$

Therefore, each term of the Euler-Lagrange equation are derived:

$$
\begin{aligned}
\frac{\partial L}{\partial \theta_{i}} & =\sum_{j=1}^{N} m_{j}\left[\dot{x}_{j} \frac{\partial \dot{x}_{j}}{\partial \theta_{i}}+\dot{y}_{j} \frac{\partial \dot{y}_{j}}{\partial \theta_{i}}-g \frac{\partial y_{j}}{\partial \theta_{i}}\right] \\
& =\sum_{j=1}^{N} h_{i j} l_{i} m_{j}\left[\dot{\theta}_{i}\left(\dot{y}_{j} \cos \theta_{i}-\dot{x}_{j} \sin \theta_{i}\right)-g \sin \theta_{i}\right] \\
& =-l_{i} \sum_{j=i}^{N} m_{j}\left[\dot{\theta}_{i} \sum_{k=1}^{j} l_{k} \dot{\theta}_{k} \sin \left(\theta_{i}-\theta_{k}\right)+g \sin \theta_{i}\right] \\
& =-g l_{i} \sin \theta_{i} \sum_{j=i}^{N} m_{j}-l_{i} \dot{\theta}_{i} \sum_{j=1}^{N} l_{j} \dot{\theta}_{j} \sin \left(\theta_{i}-\theta_{j}\right) \sum_{k=n_{i j}}^{N} m_{k}
\end{aligned}
$$

where:

$$
n_{i j}= \begin{cases}i & \text { if } j \leq i \\ j & \text { else }\end{cases}
$$

and:

$$
\begin{aligned}
\frac{\partial L}{\partial \dot{\theta}_{i}} & =l_{i} \sum_{j=i}^{N} m_{j}\left(\dot{x}_{j} \cos \theta_{i}+\dot{y}_{j} \sin \theta_{i}\right) \\
\frac{d}{d t}\left(\frac{\partial L}{\partial \dot{\theta}_{i}}\right) & =l_{i} \sum_{j=i}^{N} m_{j}\left(\ddot{x}_{j} \cos \theta_{i}-\dot{x}_{j} \dot{\theta}_{i} \sin \theta_{i}+\ddot{y}_{j} \sin \theta_{i}+\dot{y}_{j} \dot{\theta}_{i} \cos \theta_{i}\right) \\
& =l_{i} \sum_{j=i}^{N} m_{j} \sum_{k=1}^{j} l_{k}\left[\ddot{\theta}_{k} \cos \left(\theta_{i}-\theta_{k}\right)+\left(\dot{\theta}_{k}^{2}-\dot{\theta}_{i} \dot{\theta}_{k}\right) \sin \left(\theta_{i}-\theta_{k}\right)\right] \\
& =l_{i} \sum_{j=i}^{N} l_{j}\left[\ddot{\theta}_{k} \cos \left(\theta_{i}-\theta_{k}\right)+\left(\dot{\theta}_{k}^{2}-\dot{\theta}_{i} \dot{\theta}_{k}\right) \sin \left(\theta_{i}-\theta_{k}\right)\right] \sum_{k=n_{i j}}^{N} m_{k}
\end{aligned}
$$

Substituting these derived terms to the Euler-Lagrangian equation gives us:

$$
-g l_{i} \sin \theta_{i} \sum_{j=i}^{N} m_{j}-l_{i} \sum_{j=i}^{N} l_{j}\left[\ddot{\theta}_{k} \cos \left(\theta_{i}-\theta_{k}\right)+\dot{\theta}_{k}^{2} \sin \left(\theta_{i}-\theta_{k}\right)\right] \sum_{k=n_{i j}}^{N} m_{k}=Q_{i}
$$


where:

$$
Q_{i}=-k_{i}\left(\theta_{i}-\theta_{i-1}\right)+k_{i}\left(\theta_{i+1}-\theta_{i}\right)-b_{i}\left(\dot{\theta}_{i}-\dot{\theta}_{i-1}\right)+b_{i}\left(\dot{\theta}_{i+1}-\dot{\theta}_{i}\right)
$$

with $1 \leq i \leq N$. Note that $\theta_{0}$ and $\dot{\theta}_{0}$ are both zero, since the first pendulum was attached to the fixed point in space.

As a result, in the general linear equation form $\boldsymbol{A x}=\boldsymbol{b}$, the $N$ nonlinear equations of motion of the multi-pendulum model can be written as:

$$
\begin{aligned}
\sum_{j=1}^{N} a_{i j} \ddot{\theta}_{j} & =b_{i}, \quad \text { where } \\
a_{i j} & =l_{i} l_{j} \cos \left(\theta_{i}-\theta_{j}\right) \sum_{k=n_{i j}}^{N} m_{k} \\
b_{i} & =-l_{i} g \sin \theta_{i} \sum_{j=i}^{N} m_{j}-l_{i} \sum_{j=1}^{N} l_{j}\left[\dot{\theta}_{j}{ }^{2} \sin \left(\theta_{i}-\theta_{j}\right)\right] \sum_{k=n_{i j}}^{N} m_{k}-Q_{i}
\end{aligned}
$$




\section{A.2 Linearization of the Multi-Pendulum Model}

Let the lumped parameter of the model be homogenous, i.e. the mass $m_{i}$, length $l_{i}$, torsional spring coefficient $k_{i}$ and torsional damping coefficient $b_{i}$ are common for the $N$ pendula. Let these common parameters be denoted as $m, l, k$ and $b$, respectively. With the small angle approximation, the trigonometry functions can be simplified as:

$$
\theta \approx 0 \Longrightarrow \sin (\theta) \approx \theta, \quad \cos (\theta) \approx 1
$$

By linearizing the nonlinear Euler-Lagrange equation of motion about rest in the vertically-downward position, the equations can be simplified and formulated as a vector form equation:

$$
\boldsymbol{M}_{\boldsymbol{w}}^{\prime} \ddot{\boldsymbol{\theta}}+\boldsymbol{B}_{w}^{\prime} \dot{\boldsymbol{\theta}}+\left(\boldsymbol{K}_{w}^{\prime}+\boldsymbol{G}_{\boldsymbol{w}}^{\prime}\right) \boldsymbol{\theta}=\mathbf{0}
$$

where $\boldsymbol{M}_{\boldsymbol{w}}^{\prime}, \boldsymbol{B}_{w}^{\prime}, \boldsymbol{K}_{w}^{\prime}, \boldsymbol{G}_{\boldsymbol{w}}^{\prime} \in \mathbb{R}^{N \times N}$ are matrices of inertia, damping, stiffness and gravitational effects, respectively. Note that the matrix for gravitational effect was included in the stiffness term, since the gravitational force acted as a restoring force to the multi-pendulum model. 
Detailed values for each matrices are [Braun, 2003]:

\begin{tabular}{|c|c|c|c|c|c|}
\hline & $N$ & $N-1$ & $N-2$ & $\cdots$ & 1 \\
\hline & $N-1$ & $N-1$ & $N-2$ & $\ldots$ & 1 \\
\hline $\boldsymbol{M}_{\boldsymbol{w}}^{\prime}=m l^{2}$ & $N-2$ & $N-2$ & $N-2$ & $\ldots$ & 1 \\
\hline & $\vdots$ & $\vdots$ & $\vdots$ & $\ddots$ & $\vdots$ \\
\hline & 1 & 1 & 1 & $\ldots$ & 1 \\
\hline & $N$ & 0 & 0 & $\ldots$ & 0 \\
\hline & 0 & $N-1$ & 0 & $\ldots$ & 0 \\
\hline $\boldsymbol{G}_{\boldsymbol{w}}^{\prime}=m g l$ & 0 & 0 & $N-2$ & $\ldots$ & 0 \\
\hline & $\vdots$ & $\vdots$ & $\vdots$ & $\ddots$ & $\vdots$ \\
\hline & 0 & 0 & 0 & $\ldots$ & 1 \\
\hline & 2 & -1 & 0 & $\ldots$ & 0 \\
\hline & -1 & 2 & -1 & $\ldots$ & 0 \\
\hline $\boldsymbol{K}_{\boldsymbol{w}}^{\prime}=k$ & 0 & -1 & 2 & $\ldots$ & 0 \\
\hline & $\vdots$ & $\vdots$ & $\vdots$ & $\ddots$ & $\vdots$ \\
\hline & 0 & 0 & 0 & $\ldots$ & 1 \\
\hline & 2 & -1 & 0 & $\ldots$ & 0 \\
\hline & -1 & 2 & -1 & $\ldots$ & 0 \\
\hline $\boldsymbol{B}_{\boldsymbol{w}}^{\prime}=b$ & 0 & -1 & 2 & $\ldots$ & 0 \\
\hline & $\vdots$ & $\vdots$ & $\vdots$ & $\ddots$ & $\vdots$ \\
\hline & 0 & 0 & 0 & $\ldots$ & 1 \\
\hline
\end{tabular}




\section{A.3 Coordinate Transformation}

The generalized coordinate can be interchanged between absolute angle coordinates and relative angle coordinates, by simply multiplying the appropriate coordinate transformation matrix. Let the generalized coordinate vector be the relative angle coordinate $\boldsymbol{q}(t)$. The relation between the absolute angles and the relative angles of the $N$-node multi-pendulum can be written as:

$$
\left[\begin{array}{c}
\theta_{1} \\
\theta_{2} \\
\theta_{3} \\
\vdots \\
\theta_{N}
\end{array}\right]=\left[\begin{array}{ccccc}
1 & 0 & 0 & \ldots & 0 \\
1 & 1 & 0 & \ldots & 0 \\
1 & 1 & 1 & \ldots & 0 \\
\vdots & \vdots & \vdots & \ddots & \vdots \\
1 & 1 & 1 & \ldots & 1
\end{array}\right]\left[\begin{array}{c}
q_{1} \\
q_{2} \\
q_{3} \\
\vdots \\
q_{N}
\end{array}\right]:=\boldsymbol{R} \boldsymbol{q}
$$

where $\boldsymbol{R}$ denotes the coordinate transformation matrix. Reformulating the equation of motion with respect to the relative angle coordinate vector $\boldsymbol{q}$ results in:

$$
\boldsymbol{M}_{\boldsymbol{w}} \ddot{\boldsymbol{q}}+\boldsymbol{B}_{\boldsymbol{w}} \dot{\boldsymbol{q}}+\left(\boldsymbol{K}_{\boldsymbol{w}}+\boldsymbol{G}_{\boldsymbol{w}}\right) \boldsymbol{q}=\mathbf{0}
$$


Detailed values for each new matrices are:

\begin{tabular}{|c|c|c|c|c|c|}
\hline & $\sum_{i=1}^{N} i$ & $\sum_{i=1}^{N-1} i$ & $\sum_{i=1}^{N-2} i$ & $\cdots$ & 1 \\
\hline & $\sum_{i=1}^{N} i-1$ & $\sum_{i=1}^{N-1} i$ & $\sum_{i=1}^{N-2} i$ & $\cdots$ & 1 \\
\hline $\boldsymbol{M}_{\boldsymbol{w}}=m l^{2}$ & $\sum_{i=1}^{N} i-3$ & $\sum_{i=1}^{N-1} i-1$ & $\sum_{i=1}^{N-2} i$ & $\cdots$ & 1 \\
\hline & $\vdots$ & $\vdots$ & $\vdots$ & $\ddots$ & $\vdots$ \\
\hline & $N$ & $N-1$ & $N-2$ & $\cdots$ & 1 \\
\hline & $N$ & 0 & 0 & $\ldots$ & 0 \\
\hline & $N-1$ & $N-1$ & 0 & $\ldots$ & 0 \\
\hline $\boldsymbol{G}_{\boldsymbol{w}}=m g l$ & $N-2$ & $N-2$ & $N-2$ & $\ldots$ & 0 \\
\hline & $\vdots$ & $\vdots$ & $\vdots$ & $\cdot$ & $\vdots$ \\
\hline & 1 & 1 & 0 & $\cdots$ & 1 \\
\hline & 1 & -1 & 0 & $\cdots$ & 0 \\
\hline & 0 & 1 & -1 & $\ldots$ & 0 \\
\hline $\boldsymbol{K}_{\boldsymbol{w}}=k$ & 0 & 0 & 1 & $\ldots$ & 0 \\
\hline & $\vdots$ & $\vdots$ & $\vdots$ & $\cdot$ & $\vdots$ \\
\hline & 0 & 0 & 0 & $\ldots$ & 1 \\
\hline & 1 & -1 & 0 & $\cdots$ & 0 \\
\hline & 0 & 1 & -1 & $\ldots$ & 0 \\
\hline $\boldsymbol{B}_{\boldsymbol{w}}=b$ & 0 & 0 & 1 & $\cdots$ & 0 \\
\hline & $\vdots$ & $\vdots$ & $\vdots$ & $\cdot$ & $\vdots$ \\
\hline & 0 & 0 & 0 & $\ldots$ & 1 \\
\hline
\end{tabular}


The state-space representation of the linearized equation of motion can be written as:

$$
\dot{x}=A x:=\left[\begin{array}{cc}
0_{n \times n} & I_{n \times n} \\
-M_{w}^{-1}\left(K_{w}+G_{w}\right) & -M_{w}^{-1} B_{w}
\end{array}\right]\left[\begin{array}{c}
q \\
\dot{q}
\end{array}\right]
$$

where $\mathbf{0}_{\boldsymbol{n} \times \boldsymbol{n}}, \boldsymbol{I}_{\boldsymbol{n} \times \boldsymbol{n}} \in \mathbb{R}^{n \times n}$ are the zero and identity matrices, $\boldsymbol{A} \in \mathbb{R}^{2 n \times 2 n}$ is the state matrix and $\boldsymbol{x} \in \mathbb{R}^{2 n}$ is the state vector. Note that the inverse of inertia matrix $\boldsymbol{M}_{\boldsymbol{w}}$ always exist since it is a positive-definite matrix. The eigenvalues and eigenvectors of the state matrix $\boldsymbol{A}$ determine the characteristic dynamics of the $N$-node multipendulum model.

It is worth noting that the eigenvalues of state matrix $\boldsymbol{A}$ do not depend on the choice of coordinate, meaning that the eigenvalue of matrix $\boldsymbol{A}$ are equivalent for absolute angle coordinates and relative angle coordinates. 


\section{Appendix B}

\section{Calculus of Variations}

Calculus of variations is a field of mathematical analysis that calculates and finds the maxima/minima of functionals. Functional is a mathematical mapping from a set of functions to a real number, compared to function which maps from a single element to an element. Some core concepts of calculus of variations are necessary for deriving the minimum-jerk-trajectory. Hence a brief overview which offers us the basic toolset for the derivation is addressed.

\section{B.1 Problem Statement}

Assume our objective is to find a specific function $x(t)$ which yields the minimum or maximum of some functional value in interest, $V$. If function $x(t)$ is defined over some interval of domain $\left[t_{i}, t_{f}\right]$, the functional value $V$ can be formulated as following:

$$
V\{x(t)\}=\int_{t_{i}}^{t_{f}} L\left(x, \dot{x}, \ddot{x}, \cdots, x^{(n)}\right) d t
$$

where the integrand $L(\cdot)$ denotes an algebraic function which depends on function $x(t)$ and its time-derivatives.

The main goal of calculus of variations is to identify function $x(t)$ which minimizes 
or maximizes value $V$ :

$$
x(t)=\underset{x(t)}{\operatorname{argmin}} \int_{t_{i}}^{t_{f}} L\left(x, \dot{x}, \ddot{x}, \cdots, x^{(n)}\right) d t
$$

\section{B.2 Euler-Lagrange Equation}

General approach for finding function $x(t)$ which maps to the extremum of value $V$ starts with the derivation of Euler-Lagrange equation. Consider the case where our quantity of interest $V$ is solely dependent on function $x(t)$ and its first-order time derivative $\dot{x}(t)$ :

$$
V\{x(t)\}=\int_{t_{i}}^{t_{f}} L(x, \dot{x}) d t
$$

The change of value of $V$ due to a small variation of function $x(t)$ can be written as:

$$
\Delta V=\int_{t_{i}}^{t_{f}} L(x+\delta x, \dot{x}+\delta \dot{x}) d t-\int_{t_{i}}^{t_{f}} L(x, \dot{x}) d t
$$

where $\delta x(t)$ denotes the deviation from function $x(t)^{1}$ [Figure B-1].

For a single point of time, $\delta x$ and $\delta \dot{x}$ are independent variables. Hence, at an arbitrary point in time, Taylor expansion at the single point of integrand $L$ is possible. Expanding and neglecting the second- and higher-order terms results:

$$
\Delta V \approx \int_{t_{i}}^{t_{f}}\left[\frac{\partial L}{\partial x}(x, \dot{x}) \delta x\right] d t+\int_{t_{i}}^{t_{f}}\left[\frac{\partial L}{\partial \dot{x}}(x, \dot{x}) \delta \dot{x}\right] d t
$$

However, whereas $x(t)$ and $\dot{x}(t)$ may be independent at any arbitrary point of time (differentiation), $\delta \dot{x}(t)$ is dependent with function $\delta x(t)$ over the entire time domain (integration). Therefore, via integration by parts: ${ }^{2}$

$$
\int_{t_{i}}^{t_{f}}\left[\frac{\partial L}{\partial \dot{x}}(x, \dot{x}) \delta \dot{x}\right] d t=\left.\frac{\partial L}{\partial \dot{x}}(x, \dot{x}) \delta x\right|_{t_{i}} ^{t_{f}}-\int_{t_{i}}^{t_{f}}\left\{\frac{d}{d t}\left[\frac{\partial L}{\partial \dot{x}}(x, \dot{x})\right] \delta x\right\} d t
$$

For the predetermined start and end point of $x(t), \delta x\left(t_{i}\right)$ and $\delta x\left(t_{f}\right)$ are both zeros

\footnotetext{
${ }^{1}$ Notation $\delta$ was distinguished from $d$, since $\delta$ represented a deviation of function, not a single variable.

${ }^{2}$ One of the key points of integration by parts is that it interchanges the variable of integration.
} 
[Figure B-1]. Thus, applying this boundary condition gives us:

$$
\delta x\left(t_{i}\right)=\delta x\left(t_{f}\right)=\left.0 \Longrightarrow \frac{\partial L}{\partial x}(x, \dot{x}) \delta x\right|_{t_{i}} ^{t_{f}}=0
$$

Merging and rearranging the equation gives us:

$$
\Delta V=\int_{t_{i}}^{t_{f}}\left[\frac{\partial L}{\partial x}-\frac{d}{d t}\left(\frac{\partial L}{\partial \dot{x}}\right)\right] \delta x d t
$$

At an extremum, for any $\delta x(t)$, value $\Delta V$ should be zero. This result eventually leads us to the Euler-Lagrange equation:

$$
\Delta V=0 \Longrightarrow \frac{\partial L}{\partial x}-\frac{d}{d t}\left(\frac{\partial L}{\partial \dot{x}}\right)=0
$$

Function $x(t)$ which satisfies [Eqn. B.9] is the optimal function that maps onto the extremum value of $V$.

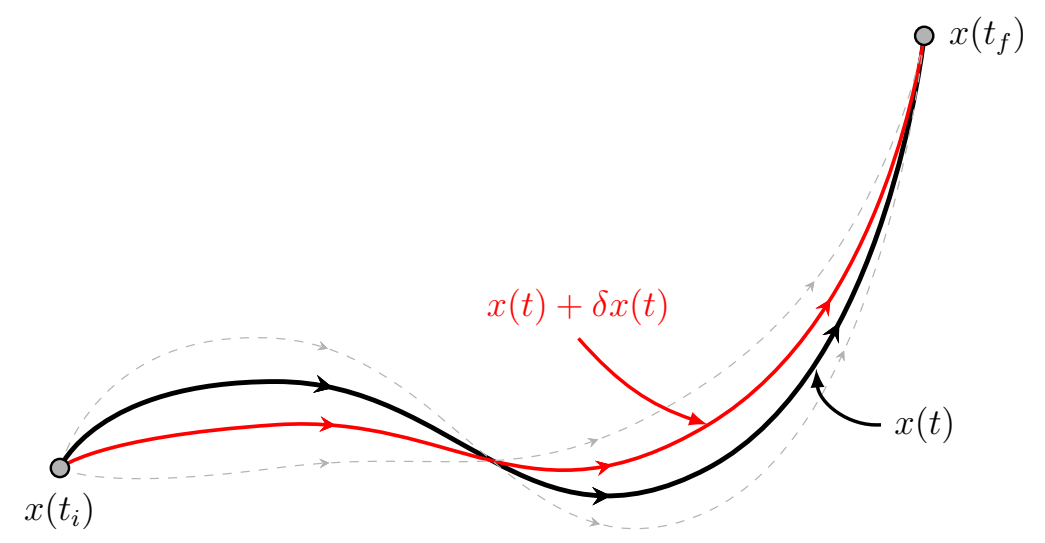

Figure B-1: Diagram of possible paths of $x(t)$ from point $x\left(t_{i}\right)$ to point $x\left(t_{f}\right)$. Black line represents one of the possible paths which $x(t)$ could take, and red line denotes the deviated path from function $x(t)$ by function $\delta x(t)$. Note that all possible paths start from the same point, $x\left(t_{i}\right)$ and end at the same point, $x\left(t_{f}\right)$, i.e. $\delta x\left(t_{i}\right)=\delta x\left(t_{f}\right)=0$. 


\section{B.3 Euler-Poisson Equation}

It is able to extend and derive a much generalized equation for cases where integrand $L$ depends on higher-order derivatives:

$$
V\{x(t)\}=\int_{t_{i}}^{t_{f}} L\left(x, \dot{x}, \ddot{x} \cdots x^{(n)}\right) d t
$$

Equivalent with the Euler-Lagrange equation, using integration by parts, it is straightforward to derive the much generalized Euler-Poisson equation:

$$
\Delta V=0 \Longrightarrow \frac{\partial L}{\partial x}-\frac{d}{d t}\left(\frac{\partial L}{\partial \dot{x}}\right)+\frac{d^{2}}{d t^{2}}\left(\frac{\partial L}{\partial \ddot{x}}\right)+\cdots+(-1)^{n} \frac{d^{n}}{d t^{n}}\left(\frac{\partial L}{\partial x^{(n)}}\right)=0
$$

The Euler-Poisson equation is the essence for deriving the minimum-jerk-trajectory.

\section{B.4 Derivation of Minimum-Jerk-Trajectory}

Humans are known to generate the "smoothest" motion to transport a limb from one posture to another [Morasso, 1981, Hogan, 1982, Flash and Hogan, 1985]. For a discrete point-to-point reaching movement, the salient observation to be modeled is that the movements exhibit a stereotyped unimodal speed profile: movement starting from rest (zero speed), rising smoothly to a single peak, then decline smoothly to rest again.

One of the mathematical models which competently describes this commonlyobserved unimodal speed profile is known as minimum-jerk-motion. Maximizing smoothness of the motion was mathematically defined as minimizing the mean-squared jerk of the movement, where jerk denoted a physical quantity defined as a third time derivative of position.

Casting it as an optimization problem, the mean-squared jerk value of the finiteduration trajectory is minimized. The cost function $V$ is:

$$
V\{x(t)\}=\frac{1}{t_{f}-t_{i}} \int_{t_{i}}^{t_{f}}\left(\frac{d^{3} x}{d t^{3}}\right)^{2} d t
$$


where $x(t)$ is the single degree-of-freedom trajectory defined in the domain $\left[t_{i}, t_{f}\right]$; scalar value $V$ is the mean-squared jerk value of trajectory $x(t)$ along time.

Without the loss of generality, treating $t_{i}=0$ and defining duration as $D:=t_{f}-t_{i}$, $V$ is reformulated as:

$$
V\{x(t)\}=\frac{1}{D} \int_{0}^{D}\left(\frac{d^{3} x}{d t^{3}}\right)^{2} d t
$$

Euler-Poisson equation [Eqn. B.11] gives us:

$$
\Delta V=0 \Longrightarrow \frac{\partial L}{\partial x}-\frac{d}{d t}\left(\frac{\partial L}{\partial \dot{x}}\right)+\frac{d^{2}}{d t^{2}}\left(\frac{\partial L}{\partial \ddot{x}}\right)-\frac{d^{3}}{d t^{3}}\left(\frac{\partial L}{\partial \dddot{x}}\right)=0
$$

where $L$ represents the square of the jerk of trajectory $x(t)$.

Since function $L$ is solely dependent on the third derivative of position $x(t)$ :

$$
\frac{d^{3}}{d t^{3}}\left(\frac{\partial L}{\partial \dddot{x}}\right)=0 \Longrightarrow \frac{d^{6} x}{d t^{6}}=0
$$

Therefore, trajectory $x(t)$ which minimizes value $V$ is a $5^{t h}$ order polynomial with respect to time $t$ :

$$
\frac{d^{6} x}{d t^{6}}=0 \Longrightarrow a_{0}+a_{1} t+a_{2} t^{2}+a_{3} t^{3}+a_{4} t^{4}+a_{5} t^{5}=0
$$

where $a_{0}$ through $a_{5}$ are constants determined by the boundary condition.

The choice of boundary conditions disambiguates the coefficients of trajectory $x(t)$. For a single discrete movement, the movement starts and ends with a resting posture. Hence, the velocity and acceleration of trajectory $x(t)$ are all zeros at the start $(t=0)$ and end $(t=D)$ of the movement. Assuming that the movement starts at zero and ends at $x_{i}$ and $x_{f}$, respectively, the boundary conditions of trajectory $x(t)$ are:

$$
x(0)=x_{i}, x(D)=x_{f}, \dot{x}(0)=\dot{x}(D)=0, \ddot{x}(0)=\ddot{x}(D)=0
$$

These 6 boundary conditions result in a 6 linear equations that define the 6 polynomial coefficients, $a_{0}$ through $a_{5}$. Solving the 6 equations determines the 6 coefficients, 
$a_{0} \sim a_{5}$, and the minimum-jerk-trajectory is derived:

$$
x(t)=x_{i}+\left(x_{f}-x_{i}\right) \cdot\left\{10\left(\frac{t}{D}\right)^{3}-15\left(\frac{t}{D}\right)^{4}+6\left(\frac{t}{D}\right)^{5}\right\}
$$

where subscripts $i$ and $f$ denote the initial and final positions, respectively; time $\mathrm{t}$ is defined in the domain $[0, D]$.

The velocity and position profile of trajectory $x(t)$ are plotted in [Figure B-2].
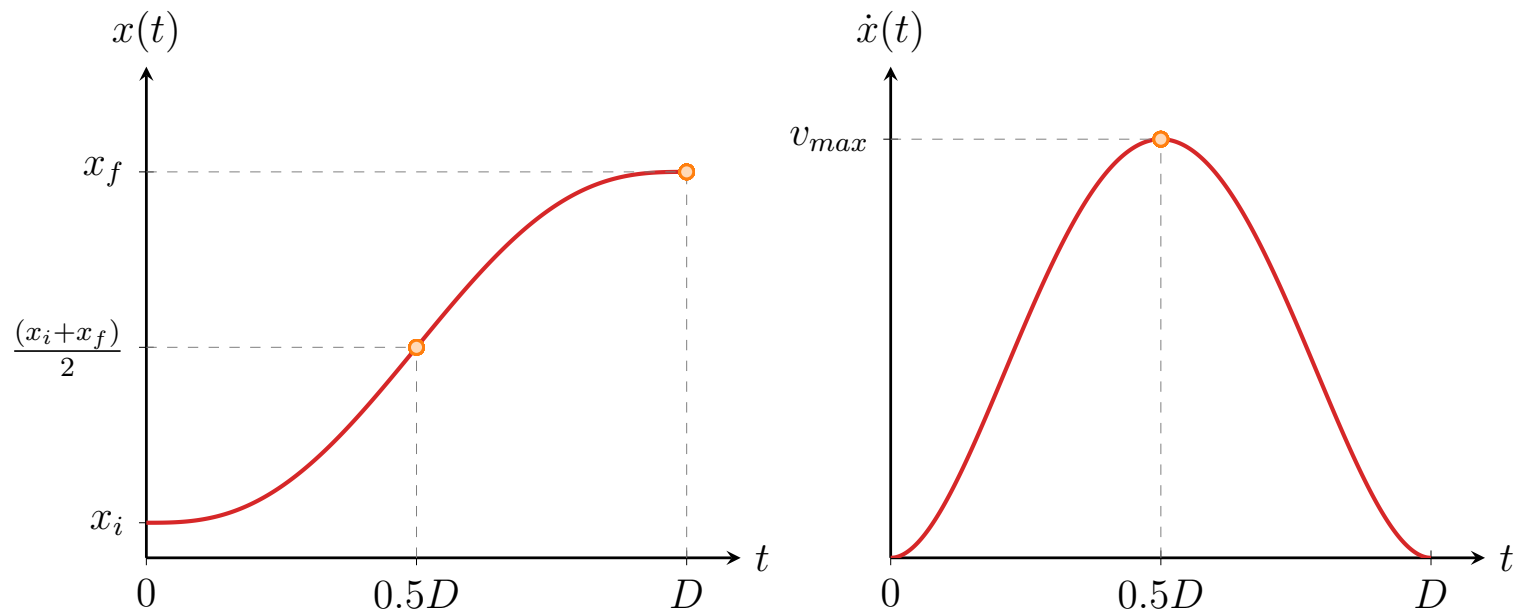

Figure B-2: Plot of position and velocity of minimum-jerk-trajectory $x(t)$ [Eqn. B.18]. A single peak unimodal bell-shaped speed profile of the minimum-jerk-trajectory, with peak value $v_{\max }$, is shown in the right. Detailed value for $v_{\max }$ was $1.875 \cdot\left(x_{f}-x_{i}\right) / D$. 


\section{Appendix C}

\section{Comparison of Nlopt Algorithms}

The nlopt (nonlinear optimization) toolbox offers multiple options of algorithm to solve the optimization problem. The algorithms are categorized by two standards: (1) whether the optimization problem is global or local and (2) whether the algorithm employs derivative-free or gradient-based approach. Within these categories, 4 global derivative-free algorithms were chosen and compared:

- ESCH (Evolutionary Algorithm)

- DIRECT-L (Dividing Rectangle - Locally Biased)

- AGS

- ISRES (Improved Stochastic Ranking Evolution Strategy)

The performance of the 4 global derivative-free algorithms: ESCH [da Silva Santos et al., 2010], DIRECT-L [Gablonsky and Kelley, 2001], AGS [Sergeyev and Markin, 1995] and ISRES [Runarsson and Yao, 2005], were compared. The simple-yet-nontrivial planar whip task [Chapter 7] was chosen for the performance evaluation. Three types of whip model were used for the comparison: short-, medium- and long-whip model [Table 7.3]. All of the optimization process comprised 600 iterations. With the given whip model, the optimal values of $L^{*}$ for each algorithms were identified and compared. 


\section{C.1 Comparison and Results}

Along the four algorithms, DIRECT-L algorithm yielded the best result (i.e. the smallest value of $L^{*}$ ) for the short-, medium- and long-whip model. The minimum values of $L^{*}$, within the 600 iterations are listed in [Table C.1]. Plots of iteration vs. $L^{*}$ value for short-, medium- and long-whip models are shown in [Figure C-1], [Figure C-2] and [Figure C-3], respectively.

\begin{tabular}{|c|c|c|c|c|}
\hline & ESCH & DIRECT_L & AGS & ISRES \\
\hline \hline short-whip & 0.0333 & 0.0323 & 0.0564 & 0.0369 \\
\hline medium-whip & 0.0520 & 0.0479 & 0.0886 & 0.1227 \\
\hline long-whip & 0.1613 & 0.1216 & 0.2500 & 0.2866 \\
\hline
\end{tabular}

Table C.1: Minimum values of $L^{*}[m]$, within the 600 iterations.

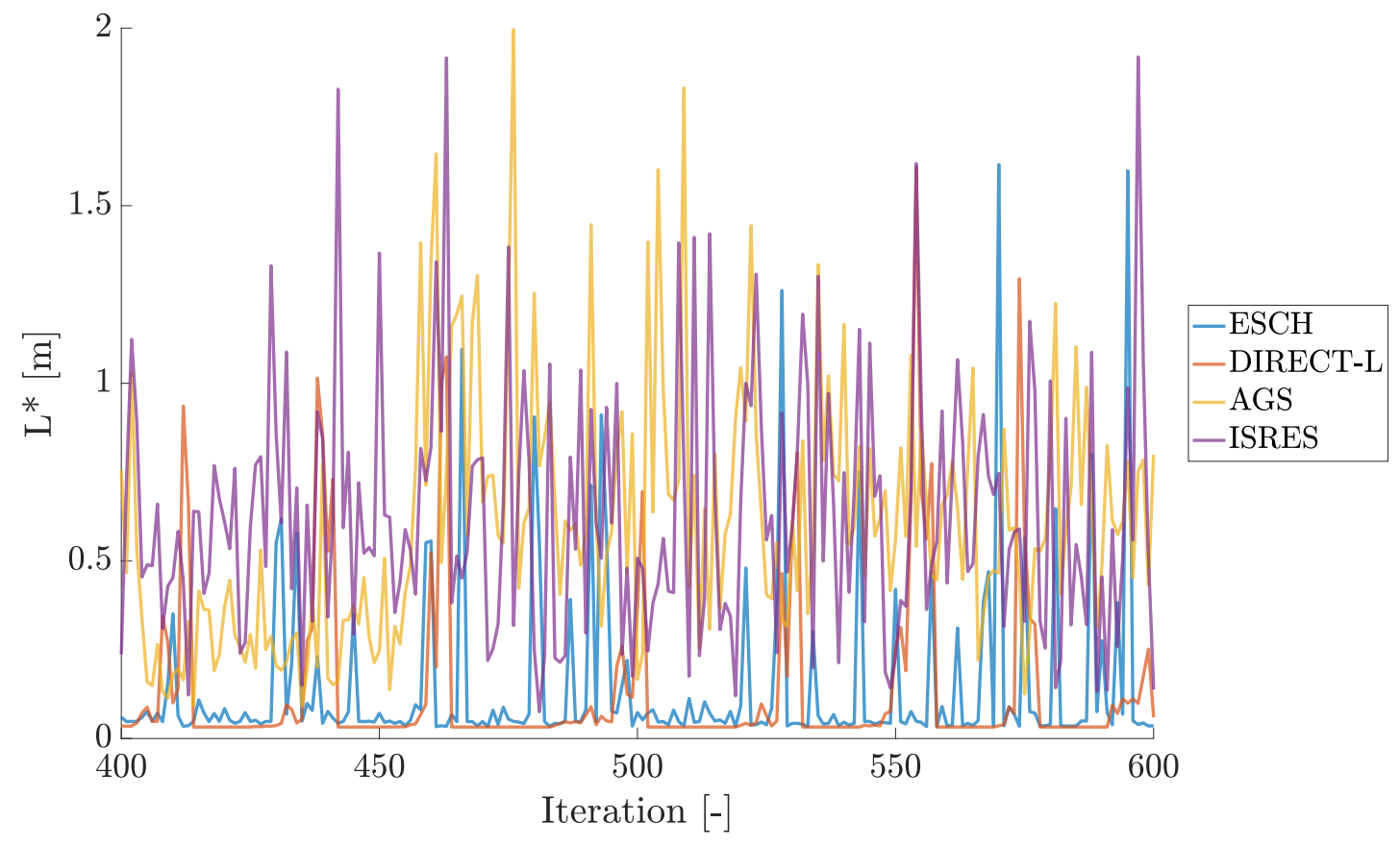

Figure C-1: Algorithm comparison: optimization with the short-whip model. Data from 400 to 600 iterations were plotted. 


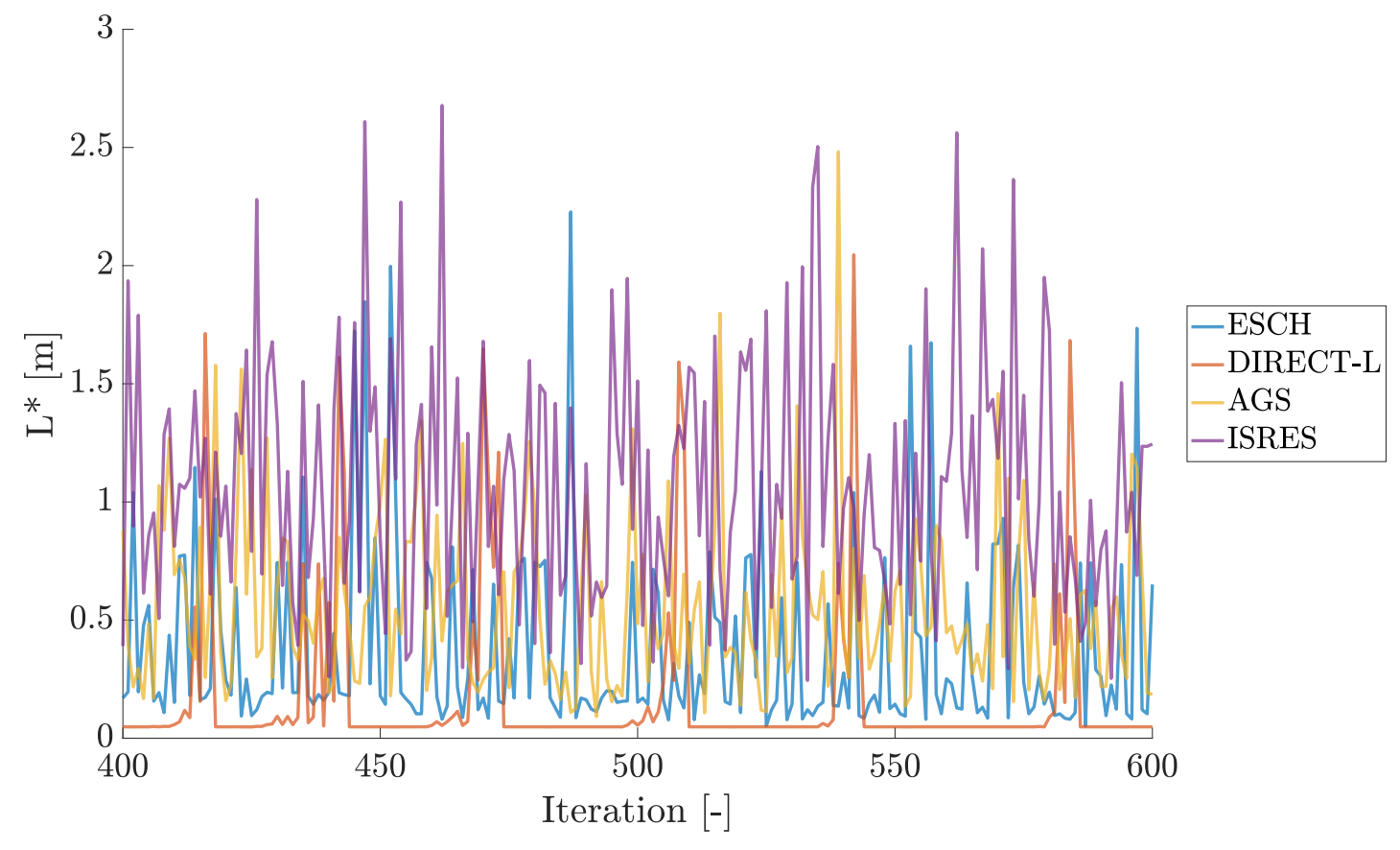

Figure C-2: Algorithm comparison: optimization with the medium-whip model. Data from 400 to 600 iterations were plotted.



Figure C-3: Algorithm comparison: optimization with the long-whip model. Data from 400 to 600 iterations were plotted. 


\section{C.2 DIRECT-L Algorithm}

The DIRECT (DIviding RECTangles) algorithm employs a pattern search method that balances local and global search within bounded constraints [Jones et al., 1993, Torczon, 1997, Gablonsky and Kelley, 2001, Jones, 2009]. Compared to algorithms which rely on randomness for optimization, DIRECT is a systematic deterministic exploration algorithm. Inspired originally from Lipschitzian optimization, which divides the bounded search space into boxes and samples the vertices of each, it is a sampling algorithm which requires no gradient information of the objective function. For the given lower and upper bound of the search space, the DIRECT algorithm divides the search space into (hyper-)rectangles in a consistent pattern. Based on the sampled data, the algorithm decides where to search next within the divided search space, and eventually searches down the optimal solution [Figure C-4]. DIRECT-L algorithm is simply the locally-biased version of DIRECT algorithm - it weights more toward the local search than the global.

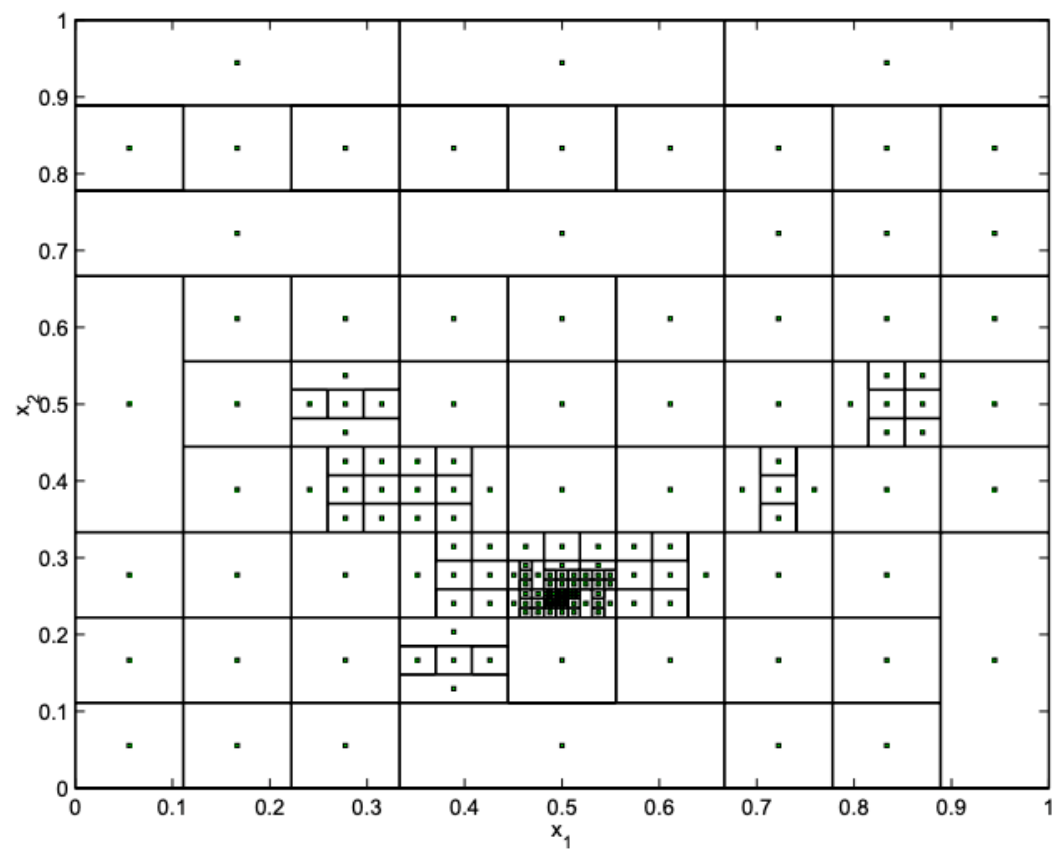

Figure C-4: Searching the optimal solution with the DIRECT algorithm in the 2dimensional search space. The optimal value for the function resided near point $\left(x_{1}, x_{2}\right)=(0.5,0.25)$. Image from [Finkel, 2003]. 


\section{Bibliography}

[Bellman, 2015] Bellman, R. E. (2015). Adaptive control processes: a guided tour, volume 2045. Princeton university press.

[Bernstein et al., 1958] Bernstein, B., Hall, D. A., and Trent, H. M. (1958). On the dynamics of a bull whip. The Journal of the Acoustical Society of America, 30(12):1112-1115.

[Berthier, 1996] Berthier, N. E. (1996). Learning to reach: a mathematical model. Developmental psychology, 32(5):811.

[Boesch and Boesch, 1990] Boesch, C. and Boesch, H. (1990). Tool use and tool making in wild chimpanzees. Folia Primatologica, 54(1-2):86-99.

[Borutzky, 2011] Borutzky, W. (2011). Bond graph modelling of engineering systems, volume 103. Springer.

[Braun, 2003] Braun, M. (2003). On some properties of the multiple pendulum. Archive of Applied Mechanics, 72(11-12):899-910.

[Brown, 1911] Brown, T. G. (1911). The intrinsic factors in the act of progression in the mammal. Proceedings of the Royal Society of London. Series B, Containing Papers of a Biological Character, 84(572):308-319.

[Brown, 1914] Brown, T. G. (1914). On the nature of the fundamental activity of the nervous centres; together with an analysis of the conditioning of rhythmic activity in progression, and a theory of the evolution of function in the nervous system. The Journal of physiology, 48(1):18-46.

[Crevecoeur et al., 2010] Crevecoeur, F., McIntyre, J., Thonnard, J.-L., and Lefevre, P. (2010). Movement stability under uncertain internal models of dynamics. Journal of Neurophysiology, 104(3):1301-1313.

[da Silva Santos et al., 2010] da Silva Santos, C. H., Goncalves, M. S., and Hernandez-Figueroa, H. E. (2010). Designing novel photonic devices by bio-inspired computing. IEEE Photonics Technology Letters, 22(15):1177-1179.

[Degallier and Ijspeert, 2010] Degallier, S. and Ijspeert, A. (2010). Modeling discrete and rhythmic movements through motor primitives: a review. Biological Cybernetics, 103(4):319-338. 
[Dominici et al., 2011] Dominici, N., Ivanenko, Y. P., Cappellini, G., d'Avella, A., Mondi, V., Cicchese, M., Fabiano, A., Silei, T., Di Paolo, A., Giannini, C., et al. (2011). Locomotor primitives in newborn babies and their development. Science, 334(6058):997-999.

[Finkel, 2003] Finkel, D. (2003). Direct optimization algorithm user guide. Technical report, North Carolina State University. Center for Research in Scientific Computation.

[Finkel and Kelley, 2004] Finkel, D. and Kelley, C. (2004). An adaptive restart implementation of direct. In International Conference on Continuous Optimization. Citeseer.

[Flanagan et al., 2003] Flanagan, J. R., Vetter, P., Johansson, R. S., and Wolpert, D. M. (2003). Prediction precedes control in motor learning. Current Biology, $13(2): 146-150$.

[Flash, 1987] Flash, T. (1987). The control of hand equilibrium trajectories in multijoint arm movements. Biological Cybernetics, 57(4-5):257-274.

[Flash and Hochner, 2005] Flash, T. and Hochner, B. (2005). Motor primitives in vertebrates and invertebrates. Current Opinion in Neurobiology, 15(6):660-666.

[Flash and Hogan, 1985] Flash, T. and Hogan, N. (1985). The coordination of arm movements: an experimentally confirmed mathematical model. Journal of Neuroscience, 5(7):1688-1703.

[Flash and Mussa-Ivaldi, 1990] Flash, T. and Mussa-Ivaldi, F. (1990). Human arm stiffness characteristics during the maintenance of posture. Experimental Brain Research, 82(2):315-326.

[Gablonsky and Kelley, 2001] Gablonsky, J. M. and Kelley, C. T. (2001). A locallybiased form of the direct algorithm. Journal of Global Optimization, 21(1):27-37.

[Gerdes and Happee, 1994] Gerdes, V. and Happee, R. (1994). The use of an internal representation in fast goal-directed movements: a modelling approach. Biological Cybernetics, 70(6):513-524.

[Goriely and McMillen, 2002] Goriely, A. and McMillen, T. (2002). Shape of a cracking whip. Physical Review Letters, 88(24):244301.

[Grillner and Wallen, 1985] Grillner, S. and Wallen, P. (1985). Central pattern generators for locomotion, with special reference to vertebrates. Annual Review of Neuroscience, 8(1):233-261.

[Hatze, 1980] Hatze, H. (1980). A mathematical model for the computational determination of parameter values of anthropomorphic segments. Journal of Biomechanics, 13(10):833-843. 
[Heaviside, 2011] Heaviside, O. (2011). Electrical Papers, volume 2. Cambridge University Press.

[Henrot, 2016] Henrot, C. C. I. (2016). Characterization of whip targeting kinematics in discrete and rhythmic tasks. Undergraduate thesis, Massachusetts Institute of Technology.

[Hermus et al., 2020] Hermus, J. R., Doeringer, J., Sternad, D., and Hogan, N. (2020). Separating neural influences from peripheral mechanics: The speedcurvature relation in mechanically-constrained actions. Journal of Neurophysiology.

[Hodgson and Hogan, 2000] Hodgson, A. J. and Hogan, N. (2000). A modelindependent definition of attractor behavior applicable to interactive tasks. IEEE Transactions on Systems, Man, and Cybernetics, Part C (Applications and Reviews), 30(1):105-118.

[Hoffer and Andreassen, 1981] Hoffer, J. and Andreassen, S. (1981). Regulation of soleus muscle stiffness in premammillary cats: intrinsic and reflex components. Journal of Neurophysiology, 45(2):267-285.

[Hogan, 1982] Hogan, N. (1982). Control and coordination of voluntary arm movements. In 1982 American Control Conference, pages 522-528. IEEE.

[Hogan, 1985] Hogan, N. (1985). Impedance control: An approach to manipulation: Part ii-implementation. Journal of Dynamic Systems, Measurement, and Control, 107(1):8-16.

[Hogan, 2013] Hogan, N. (2013). A general actuator model based on nonlinear equivalent networks. IEEE/ASME Transactions on Mechatronics, 19(6):1929-1939.

[Hogan, 2017] Hogan, N. (2017). Physical interaction via dynamic primitives. In Geometric and Numerical Foundations of Movements, pages 269-299. Springer.

[Hogan and Sternad, 2007] Hogan, N. and Sternad, D. (2007). On rhythmic and discrete movements: reflections, definitions and implications for motor control. Experimental Brain Research, 181(1):13-30.

[Hogan and Sternad, 2012] Hogan, N. and Sternad, D. (2012). Dynamic primitives of motor behavior. Biological Cybernetics, 106(11-12):727-739.

[Hogan and Sternad, 2013] Hogan, N. and Sternad, D. (2013). Dynamic primitives in the control of locomotion. Frontiers in Computational Neuroscience, 7:71.

[Hunt, 1996] Hunt, G. R. (1996). Manufacture and use of hook-tools by new caledonian crows. Nature, 379(6562):249.

[Ijspeert et al., 2002] Ijspeert, A. J., Nakanishi, J., and Schaal, S. (2002). Movement imitation with nonlinear dynamical systems in humanoid robots. In Proceedings 2002 IEEE International Conference on Robotics and Automation (Cat. No. 02CH37292), volume 2, pages 1398-1403. IEEE. 
[Johnson-Frey, 2004] Johnson-Frey, S. H. (2004). The neural bases of complex tool use in humans. Trends in Cognitive Sciences, 8(2):71-78.

[Jones, 2009] Jones, D. R. (2009). Direct global optimization algorithm. Encyclopedia of optimization, 1(1):431-440.

[Jones et al., 1993] Jones, D. R., Perttunen, C. D., and Stuckman, B. E. (1993). Lipschitzian optimization without the lipschitz constant. Journal of optimization Theory and Applications, 79(1):157-181.

[Joyce et al., 1969] Joyce, G., Rack, P., and Westbury, D. (1969). The mechanical properties of cat soleus muscle during controlled lengthening and shortening movements. The Journal of Physiology, 204(2):461-474.

[Kandel et al., 2000] Kandel, E. R., Schwartz, J. H., Jessell, T. M., of Biochemistry, D., Jessell, M. B. T., Siegelbaum, S., and Hudspeth, A. (2000). Principles of Neural Science, volume 4. McGraw-hill New York.

[Kargo and Giszter, 2008] Kargo, W. J. and Giszter, S. F. (2008). Individual premotor drive pulses, not time-varying synergies, are the units of adjustment for limb trajectories constructed in spinal cord. Journal of Neuroscience, 28(10):2409-2425.

[Kawato, 1999] Kawato, M. (1999). Internal models for motor control and trajectory planning. Current Opinion in Neurobiology, 9(6):718-727.

[Kenward et al., 2005] Kenward, B., Weir, A. A., Rutz, C., and Kacelnik, A. (2005). Behavioural ecology: Tool manufacture by naive juvenile crows. Nature, 433(7022):121.

[Khusainov et al., 2018] Khusainov, R., Klimchik, A., and Magid, E. (2018). Kinematic and dynamic approaches in gait optimization for humanoid robot locomotion. In Informatics in Control, Automation and Robotics, pages 293-320. Springer.

[Lewis, 2006] Lewis, J. W. (2006). Cortical networks related to human use of tools. The Neuroscientist, 12(3):211-231.

[Lipps et al., 2015] Lipps, D. B., Baillargeon, E. M., Ludvig, D., and Perreault, E. J. (2015). System identification of multidimensional shoulder impedance during volitional contractions. IFAC-PapersOnLine, 48(28):1369-1374.

[Martínez-del Rincón et al., 2007] Martínez-del Rincón, J., Orrite-Uruñuela, C., and Rogez, G. (2007). Rao-blackwellized particle filter for human appearance and position tracking. In Iberian Conference on Pattern Recognition and Image Analysis, pages 201-208. Springer.

[McMillen and Goriely, 2003] McMillen, T. and Goriely, A. (2003). Whip waves. Physica D: Nonlinear Phenomena, 184(1-4):192-225. 
[McNamee and Wolpert, 2019] McNamee, D. and Wolpert, D. M. (2019). Internal models in biological control. Annual Review of Control, Robotics, and Autonomous Systems, 2:339-364.

[Morasso, 1981] Morasso, P. (1981). Spatial control of arm movements. Experimental Brain Research, 42(2):223-227.

[Mottershead, 2016] Mottershead, J. E. (2016). Modern Practice in Stress and Vibration Analysis: Proceedings of the Conference Held at the University of Liverpool, 3-5 April 1989. Elsevier.

[Mussa-Ivaldi et al., 1985] Mussa-Ivaldi, F. A., Hogan, N., and Bizzi, E. (1985). Neural, mechanical, and geometric factors subserving arm posture in humans. Journal of Neuroscience, 5(10):2732-2743.

[Myers, 2009] Myers, D. G. (2009). Exploring Psychology. Macmillan.

[Nichols and Houk, 1976] Nichols, T. and Houk, J. (1976). Improvement in linearity and regulation of stiffness that results from actions of stretch reflex. journal of Neurophysiology, 39(1):119-142.

[Paine et al., 2013] Paine, N., Oh, S., and Sentis, L. (2013). Design and control considerations for high-performance series elastic actuators. IEEE/ASME Transactions on Mechatronics, 19(3):1080-1091.

[Peters and Schaal, 2006] Peters, J. and Schaal, S. (2006). Policy gradient methods for robotics. In 2006 IEEE/RSJ International Conference on Intelligent Robots and Systems, pages 2219-2225. IEEE.

[Prochazka et al., 2000] Prochazka, A., Clarac, F., Loeb, G. E., Rothwell, J. C., and Wolpaw, J. R. (2000). What do reflex and voluntary mean? modern views on an ancient debate. Experimental Brain Research, 130(4):417-432.

[Purves et al., 2004] Purves, D., Augustine, G. J., Fitzpatrick, D., Hall, W. C., LaMantia, A.-S., McNamara, J. O., and Williams, S. M. (2004). Neuroscience. Sunderland, MA: Sinauer Associates, 773.

[Rack and Westbury, 1969] Rack, P. M. and Westbury, D. (1969). The effects of length and stimulus rate on tension in the isometric cat soleus muscle. The Journal of Physiology, 204(2):443-460.

[Rohrer et al., 2004] Rohrer, B., Fasoli, S., Krebs, H. I., Volpe, B., Frontera, W. R., Stein, J., and Hogan, N. (2004). Submovements grow larger, fewer, and more blended during stroke recovery. Motor control, 8(4):472-483.

[Runarsson and Yao, 2005] Runarsson, T. P. and Yao, X. (2005). Search biases in constrained evolutionary optimization. IEEE Transactions on Systems, Man, and Cybernetics, Part C (Applications and Reviews), 35(2):233-243. 
[Sakaguchi et al., 2015] Sakaguchi, Y., Tanaka, M., and Inoue, Y. (2015). Adaptive intermittent control: a computational model explaining motor intermittency observed in human behavior. Neural Networks, 67:92-109.

[Schaal et al., 2003] Schaal, S., Peters, J., Nakanishi, J., and Ijspeert, A. (2003). Control, planning, learning, and imitation with dynamic movement primitives. In Workshop on Bilateral Paradigms on Humans and Humanoids, IEEE International Conference on Intelligent Robots and Systems, pages 1-21.

[Schaal et al., 2004] Schaal, S., Sternad, D., Osu, R., and Kawato, M. (2004). Rhythmic arm movement is not discrete. Nature Neuroscience, 7(10):1136.

[Sergeyev and Markin, 1995] Sergeyev, Y. D. and Markin, D. L. (1995). An algorithm for solving global optimization problems with nonlinear constraints. Journal of Global Optimization, 7(4):407-419.

[Shepard and Metzler, 1971] Shepard, R. N. and Metzler, J. (1971). Mental rotation of three-dimensional objects. Science, 171(3972):701-703.

[Shidara et al., 1993] Shidara, M., Kawano, K., Gomi, H., and Kawato, M. (1993). Inverse-dynamics model eye movement control by purkinje cells in the cerebellum. Nature, 365(6441):50-52.

[Shinbrot et al., 1992] Shinbrot, T., Grebogi, C., Wisdom, J., and Yorke, J. A. (1992). Chaos in a double pendulum. American Journal of Physics, 60(6):491-499.

[Sing et al., 2009] Sing, G. C., Joiner, W. M., Nanayakkara, T., Brayanov, J. B., and Smith, M. A. (2009). Primitives for motor adaptation reflect correlated neural tuning to position and velocity. Neuron, 64(4):575-589.

[Sperelakis, 2012] Sperelakis, N. (2012). Cell physiology source book: essentials of membrane biophysics. Elsevier.

[Sternad, 2008] Sternad, D. (2008). Towards a unified theory of rhythmic and discrete movements - behavioral, modeling and imaging results. In Coordination: Neural, behavioral and social dynamics, pages 105-133. Springer.

[Sternad et al., 2000] Sternad, D., Dean, W. J., and Schaal, S. (2000). Interaction of rhythmic and discrete pattern generators in single-joint movements. Human Movement Science, 19(4):627-664.

[Stulp et al., 2012] Stulp, F., Theodorou, E. A., and Schaal, S. (2012). Reinforcement learning with sequences of motion primitives for robust manipulation. IEEE Transactions on Robotics, 28(6):1360-1370.

[Thoroughman and Shadmehr, 2000] Thoroughman, K. A. and Shadmehr, R. (2000). Learning of action through adaptive combination of motor primitives. Nature, 407(6805):742. 
[Todorov et al., 2012] Todorov, E., Erez, T., and Tassa, Y. (2012). Mujoco: A physics engine for model-based control. In 2012 IEEE/RSJ International Conference on Intelligent Robots and Systems, pages 5026-5033. IEEE.

[Torczon, 1997] Torczon, V. (1997). On the convergence of pattern search algorithms. SIAM Journal on optimization, 7(1):1-25.

[von Hofsten, 1991] von Hofsten, C. (1991). Structuring of early reaching movements: a longitudinal study. Journal of motor behavior, 23(4):280-292.

[Wolpert et al., 1998] Wolpert, D. M., Miall, R. C., and Kawato, M. (1998). Internal models in the cerebellum. Trends in Cognitive Sciences, 2(9):338-347.

[Yamakawa et al., 2016] Yamakawa, Y., Odani, K., and Ishikawa, M. (2016). Sonicspeed manipulation of a bull whip using a robot manipulator. In 2016 IEEE International Conference on Advanced Intelligent Mechatronics (AIM), pages 11391144. IEEE. 